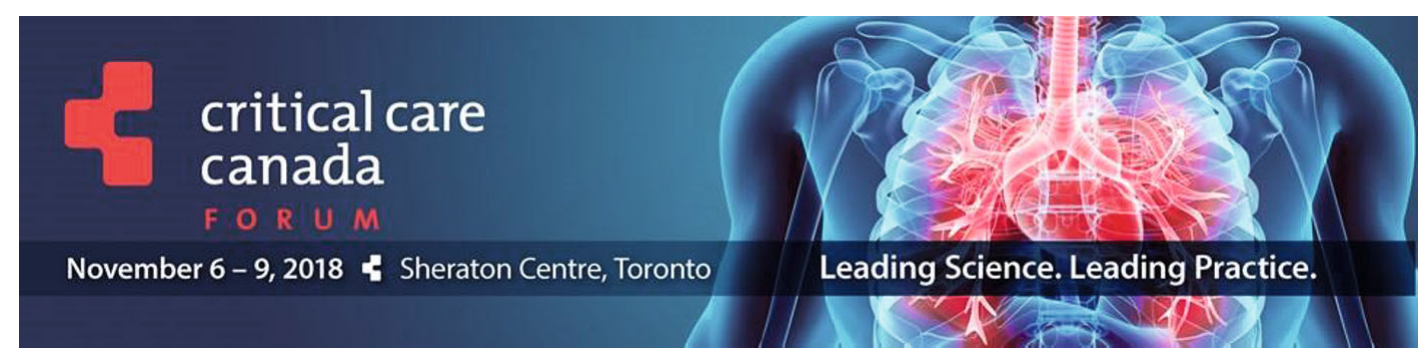

\title{
Critical Care Canada Forum 2018 Abstracts
}




\section{Contents}

"It Takes Both Sides to Build a Bridge": Patients' Family Satisfaction in a Scottish District General Hospital Intensive Care Unit

A Quality Assurance Study Assessing Mechanical Ventilation Practices in The Emergency Department

A Quality Improvement Initiative Improving Daily Extubation Rates in the ICU . 9

A Survey of Burnout in Academic Pediatric, Adult and a Community ICU in metropolitan Halifax Nova Scotia.

A Systematic Review of the Efficacy of Mannitol Versus Hypertonic Saline in the Treatment of Raised Intracranial Pressure in Adults Suffering from

Traumatic Brain Injury

AcceleratephenoTM Potential Influence on Timeliness of Management Decisions for Patients with Positive Blood Cultures

Admission Frailty Laboratory Index (FI-LAB) and Associated Mortality and Costs in Older ICU Patients with Suspected Infection

An Interprofessional and Patient-Centred Approach to Improving Transitions from Intensive Care Unit (ICU) to Ward

Cardiovascular ICU Nurses' Feedback on the Use of the CHG-Lock ${ }^{\mathrm{TM}}$ : A Survey as Part of the CHG Pilot Study.

CHG-Lock Device for Prevention of Central line Infections in the ICU - An Open

Label Randomized Pilot Study: Protocol .

Comparison of Outcomes and Costs Between Adult Diabetic Ketoacidosis

Patients Admitted to the ICU or Stepdown Unit.

Comparison of The Source and Prognostic Utility of Cfdna In Trauma and Sepsis.

Complications of Computed Tomographic Pulmonary Angiography to Rule Out Pulmonary Embolism in a Tertiary Care Cancer \& Neuroscience Center.

Determining the Optimal Time for LibEration from Renal Replacement Therapy in Critically III Patients: A Systematic Review and Meta-Analysis (DOnE RRT) 30

Development and Evaluation of an Interactive ICU Workshop for High School

Students

Diarrhea During Critical IIIness: An Interim Analysis .......................................... 36

Diarrhea During Mechanical Ventilation: A Multicenter Study..

Do Social Determinants Increase the Risk of an In-Hospital Critical Event When Admitted to Medicine or Surgery?

Effect of Graded Passive Cycling on Cerebral Blood Flow, Cardiac Function, and Global Hemodynamics in Sepsis

Embrionic-Derived $\mathrm{Myb}^{-}$Macrophages Improve Recovery and Reduce

Bacterial Burden in Rodent Polymicrobial Abdominal Sepsis 
End of Life Skills \& Professionalism for Critical Care Residents in Training (ESPRIT): A Canada-Wide Educational Survey

Epidemiologic Risk Factors of Antimicrobial Resistance in Patients with Septic Shock Admitted to North American Critical Care Units: A Retrospective Cohort

Epidemiology of death in a single PICU over 15 years

Establishing Feasibility \& Safety of Providing Critical Care for Patients with Ebola through Design of a Simulated Ebola Treatment Unit.

Evidence Bulletins: Bringing Evidence-Based Recommendations on Donor Management to the Bedside

Factors Impacting Physician Recommendation for Tracheostomy in Pediatric Prolonged Mechanical Ventilation: A National Cross-Sectional Survey.

Humanization of Critical Care - Psychological Effects on Healthcare Professionals \& Relatives: A Systematic Review.

Identification of mechanistic pathways that lead to Disseminated Intravascular Coagulation (DIC) in Sepsis: Implications for screening

Incorporating Laboratory Data into Machine Learning Models Improves

Predictions of In-Hospital Morality After Rapid Response Team Call: A Followup Study

Inter-Rater Reliability and Responsiveness of Key Physical Functional

Outcome Measures in ICU Survivors.

Interventions to Improve Surrogate Decision-Making in Intensive Care Units: A Systematic Review and Meta-Analysis of Randomized Controlled Trials

Introducing a Compassion-Centered End of Life Project in a Large Community ICU: Facilitators and Barriers.

Medical Fragility and Inpatient Mortality in a Quaternary Care Pediatric ICU.... 73

Melatonin and Melatonin Agonists as Adjuncts for Sleep and Sedation in

Critical IIIness: A Systematic Review

Melatonin and Melatonin Agonists to Prevent and Treat Delirium in Critical

Illness: A Systematic Review

Mesenchymal Stromal/Stem Cells Enhance Bacterial Killing Partly Via

Hemoxygenase-1 Expression in Macrophages from Healthy and Septic

Patients and Accelerate Bacterial Clearance and Recovery in Preclinical Sepsis

Model

Moving to a Newly Built Adult Intensive Care Unit with Single Patient Rooms: Impact on Clinicians and ICU Performance

Optimizing Mobility for Critically III Patients Undergoing Continuous Renal Replacement Therapy: A Retrospective Cohort Study (MOvE CRRT) ................. 84

Organ and Tissue Donation Physician Leadership Model: Mapping a Way Forward. 
Organ Donation Programs and the Definition of Success: A Scoping Review

Preliminary Results

Post-Mortem Expressions of Compassion: A Secondary Qualitative Analysis of Family and Clinician Experiences of Sympathy Cards in the 3 Wishes Project 89

Practice Patterns of Phenobarbital Use in Severe Alcohol Withdrawal Syndrome

Pre-Arrest and Intra-Arrest Prognostic Factors for Survival to Discharge in InHospital Cardiac Arrest - A Systematic Review and Meta-analysis.

Predicting Time to Death After the Withdrawal of Life Supporting Therapies

Using Variability Analysis of Vital Signs Waveform Data

Predictors of Graft Loss Following Kidney Transplantation: Systematic Review and Meta-Analysis.

Prevalence and Prognosis of Critically III Patients with a Failed Cuff Leak Test: A Retrospective Observational Study

Prognostic Accuracy of the Hamilton Early Warning Score (HEWS) Among Hospitalized Patients Assessed by a Rapid Response Team .

Prognostic Accuracy of the HEART Score for Prediction of Major Adverse Cardiac Events in Patients Presenting with Chest Pain - A Systematic Review and Meta-analysis

Promoting Professional Development of Critical Care Research Coordinators through Annual Workshops 106

Quantifying the Academic Activities of Canadian Critical Care Research Coordinators Group (CCCRCG)

Survey of Canadian Intensive Care Physicians' Attitudes Towards a Presumed Consent Model for Deceased Organ Donation.

The Cost Effectiveness of Mechanical Ventilation Using PAV+ Mode in Canada

The transition of International Medical Graduate (IMG) fellows into the ICU Team: Identity Gain or Loss?

Transitions in Critical Care: Evaluating the Implementation of a Handover Tool for Postoperative Cardiac Surgery Patients

Trends and Outcomes in Mechanically Ventilated Cirrhotic Patients. 119

Understanding the Influence of Inter-Professional Relational Networks within Organ Donation Programs in Ontario: A Research Protocol and Preliminary Results

Using Non-Invasive Techniques to Explore Personalized Cerebral Perfusion Targets in Critically III Patients 
“It Takes Both Sides to Build a Bridge": Patients' Family Satisfaction in a Scottish District General Hospital Intensive Care Unit

Mautsi, Chenai ${ }^{1}$

1 Intensive care, Hairmyres Hospital, Lanarkshire, Scotland

Introduction: There is limited research into patients' family satisfaction levels in Intensive Care Units within the United Kingdom. Family feedback is essential to achieve holistic patient centered care and can identify overlooked service improvements.

Methods: This quality improvement project was completed in a 10 bedded ICU in a Scottish District General Hospital over 1 month. Participants were given a validated patient family satisfaction survey, FS-ICU 24, prior to the patients' discharge or approached 4 weeks later if the patient had died in ICU. Quantitative data: the fivepoint Likert scale responses were converted to percentage scores; higher values representing better satisfaction. Qualitative data: free text responses were analysed using a two-tier system; grouped into themes and then satisfied or dissatisfied responses.

Results: 25 surveys were collected in total with a $96 \%$ completion rate. Patient or family demographics did not alter the satisfaction rating. Average satisfaction of care and decision-making was $90.1 \%$ and $80.8 \%$ respectively. $50 \%$ of families felt they were neither in control nor out of control over the care of their family member. With regards to decision making, $37.5 \%$ felt they were neither included or excluded, and $8 \%$ felt overwhelmed. In the quantitative analysis there were three main themes of dissatisfaction: transition from ICU to non-specialist care, mental health awareness and management, and family involvement in personal care.

Conclusion: Most families were highly satisfied with ICU care received by the patient. However, three specific areas have been identified to improve the department. Our action plan is as follows: (1) a formal ICU aftercare package (2) family involvement in personal care and (3) assessment of premorbid and inpatient mental health (including a collateral history).

Fig 1 Overall satisfaction score in each domain; care and decision making

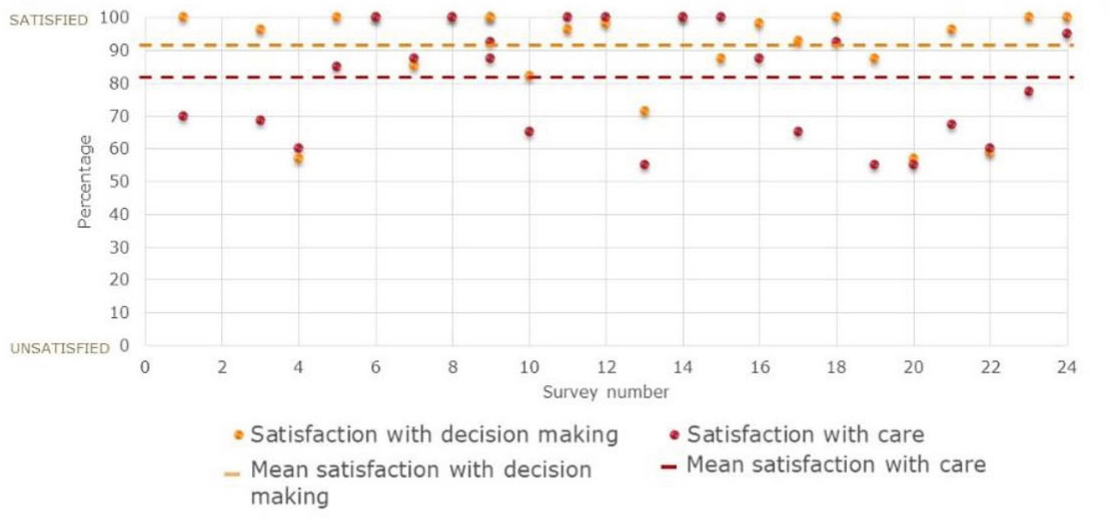




\section{References}

1. Wall RJ, et al.: Refinement, scoring, and validation of the Family Satisfaction in the Intensive Care Unit (FS-ICU) survey. Crit Care Med 2007, 35: 271-279. 
A Quality Assurance Study Assessing Mechanical Ventilation Practices in The Emergency Department

Skitch, Steven ${ }^{1}$; Rochwerg, Bram²

1 Department of Critical Care Medicine, Joseph Brant Memoral Hospital, Burlington, Ontario, Canada

2 Department of Medicine (Division of Critical Care), Department of Health Research Methods, Evidence and Impact, McMaster University, Hamilton, Ontario, Canada

Introduction: Mechanical ventilation (MV) is lifesaving for patients with respiratory failure and is frequently initiated in the emergency department (ED). Despite the benefits, MV can be associated with complications. Ventilator settings affects the risk of developing ventilator-associated complications and subsequent outcomes. Specifically, both low tidal volume (TV) ventilation and avoidance of excess oxygen delivery have been associated with improved outcomes for patients receiving MV. Recent research indicates that these benefits extend to ED patients. Specifically, a before-after study examined the impact of an ED bundle that focused on low TV ventilation, levels of PEEP, and oxygen weaning. The bundle was associated with numerous benefits including lower rates of ARDS, shorter durations of MV, and a reduction in mortality. This result warrants replication to determine if an ED MV bundle can improve outcomes.

Objectives: 1) To document current ventilation practices through a retrospective chart review of patients intubated in the ED and transferred to the ICU; 2) To analyze the association between ED ventilation settings and subsequent ICU outcomes

Methods: We identified consecutive ventilated ED patients at the study centre over a 1 -year period. Demographics, ED length of stay, diagnosis, mode of arrival, and indication for intubation were abstracted. ED ventilation settings including delivered TV, PEEP, percentage inspired oxygen (FiO2), and oxygen saturations were also collected. Lung protective ventilation (LPV) was defined as a TV of less than or equal to $8 \mathrm{ml} / \mathrm{kg}$ of predicted body weight given this is the upper limit prescribed in ARDSnet. In line with previous research, hyperoxia was defined as the provision of an $\mathrm{FiO} 2$ greater than $40 \%$ with saturations greater than $95 \%$. Analysis were conducted examining the association between ED ventilation practices and ICU outcomes. The primary outcome was mortality during the index hospitalization. Secondary outcomes included the duration of MV among survivors and the mean arterial oxygen to inspired oxygen ratio (PaO2/FiO2) during the initial 48 hours of admission.

Results: 126 patients were included in the study. The mean time from ED arrival to intubation was 3.1 hours (SD $=6.8$ hours) and from intubation to ICU transfer was 3.5 hours (SD -2.1 hours). The majority (99\%) of patients received a PEEP of least $5 \mathrm{~cm}$ of water in the ED. Only $20 \%$ of patients had an ideal body weight recorded in the ED and $40 \%$ of patients were ventilated with volumes greater than $8 \mathrm{ml} / \mathrm{kg}$. Forty-eight percent of patients met criteria for hyperoxia while ventilated in the ED. Thirty-one percent of patients who received LPV died during the index hospitalization as compared to $42 \%$ percent of patients who did not receive LPV which was not a statistically significant difference. Patients who met criteria fo hyperoxia in the ED were more likely to die ( $45 \%$ mortality) compared with patients who did not receive hyperoxia in the ED ( $26 \%$ mortality; RR $=1.74,95 \%$ Cl $1.04-2.92$ ).

Conclusions: The current study indicates that many patients are ventilated in the ED using tidal volumes and levels of $\mathrm{FiO} 2$ greater than what is recommended based upon research and guidelines. These unadjusted results suggest that the delivery of excessive oxygen in the ED may negatively impact patient outcomes. A quality improvement initiative focused on optimizing ED ventilation has the potential to change practice and improve outcomes. 


\section{References}

1 Slutsky AS, Ranieri VM. Ventilator-Induced Lung Injury. N Engl J Med. 2013;369(22):2126-2136. Acute Respiratory Distress Syndrome Network. Ventlation with lower tidal volumes as compared with traditional tidal volumes for acute lung injury and the acute respiratory distress syndrome. $\mathrm{N}$ Engl J Med. 2000;342(18):1301-1308.

2 Fan E, Del Sorbo L, Goligher EC, et al. An official American Thoracic Society/European Society of intensive care medicine/society of critical care medicine clinical practice guideline: Mechanical ventilation in adult patients with acute respiratory distress syndrome. Am J Respir Crit Care Med. 2017;195(9):12531263.

3 Serpa Neto A, Cardoso SO, Manetta JA, et al. Association between use of lungprotective ventilation with lower tidal volumes and clinical outcomes among patients without acute respiratory distress syndrome. JAMA. 2012;308(16):1651-1659.

4 Fuller BM, Ferguson IT, Mohr NM, et al. Lung-Protective Ventilation Initiated in the Emergency Department (LOV-ED): A Quasi-Experimental, Before-After Trial. Ann Emerg Med. 2016;(March).

5 Fuller BM, Mohr NM, Dettmer M, et al. Mechanical ventilation and acute lung injury in emergency department patients with severe sepsis and septic shock: An observational study. Acad Emerg Med. 2013;20(7):659-669.

6 Fuller BM, Mohr NM, Miller CN, et al. Mechanical ventilation and ARDS in the ED: A multicenter, observational, prospective, cross-sectional study. Chest. 2015;148(2):365-374.

7 Worster A, Bledsoe RD, Cleve P, Fernandes CM, Upadhye S, Eva K. Reassessing the methods of medical record review studies in emergency medicine research. Ann Emerg Med. 2005;45(4):448-451. 


\section{A Quality Improvement Initiative Improving Daily Extubation Rates in the ICU}

Campbell, Vagia $^{1}$

1 Respiratory Therapy - Critical Care, Sinai Health System - Mount Sinai Hospital, Toronto, Ontario, Canada

Introduction: Mechanical ventilation (MV), a life sustaining treatment, is associated with complications leading to worsened patient outcomes. [1-6] Unnecessary risks associated with prolonged MV can be mitigated by reducing the duration of MV. [7-8] The aim of this study was to increase the daily E-R in patients who meet criteria, by $20 \%$ over a 10 -month period in the ICU at Mount Sinai Hospital, Toronto, Canada. Methods: Using quality improvement methodology, key drivers were identified which enable extubation. A solution-based process map generated change initiatives which were rolled out over a 13-week time series study; they included: Readiness

Assessment Checklist, Earlier Assessment, Registered Respiratory Therapists (RRT) Physician Extubation Huddle, and Medical Directive for RRT to Extubate.

Results: The primary outcome of a $20 \%$ improvement in E-R was not achieved, yet a $13 \%$ increase was noted (Table1). Change idea compliance was very good for patient readiness and assessment; however inconsistent for the initiatives targeting medical orders. The Medical Directive initiative was not yet rolled out at the time of print. There was no change in Re-Intubation rates.

Table 1: Family of Measures

\begin{tabular}{|l|l|l|}
\hline Outcome Measures & Extubation Rate & $13 \%$ improvement \\
\hline & Mechanical Ventilation Days & $\begin{array}{l}\text { Increasing trend, unstable } \\
\text { baseline }\end{array}$ \\
\hline Process Measures & Readiness Assessment Checklist & $100 \%$ compliance \\
\hline & Earlier Assessment & $94 \%$ compliance \\
\hline & RRT - Physician Extubation Huddle & $58.3 \%$ compliance \\
\hline & Medical Directive for RRT to & Not rolled out \\
\hline & Extubate & No special cause variation \\
\hline Balancing Measures & Re-Intubation Rates & No special cause variation \\
\hline & Self-Extubation Rate &
\end{tabular}

Conclusion: The change ideas hypothesized to have the greatest impact on $E-R$ were not successfully rolled out. A better understanding of the distribution of the root cause to extubation delay may have resulted in more focused interventions. Additionally, the use of Spontaneous Breathing Trials as readiness to extubate criteria may not be appropriate for all subset of patients. Future work should center on standardization of extubation criteria.

\section{References}

1. Kiekkas P et al. Nurs Crit Care. 2013;18(3):123-134.

2. De Groot RI et al. Crit Care. 2011;15(January):R19.

3. Finfer SR et al. N Engl J Med. 2013;22369(28):2126-2136.

4. Tremblay LN et al. Intensive Care Med. 2006;32(1):24-33.

5. Auerbach DI et al. N Engl J Med. 2013;368(16):1470-1472.

6. Chastre J et al. Am J Respir Crit Care Med. 2002;165(23):867-903.

7. Coplin WM et al. Am J Respir Crit Care Med. 2000.

8. Maclntyre NR. Respir Care. 2013;58(6):1074-1082. 


\section{A Survey of Burnout in Academic Pediatric, Adult and a Community ICU in metropolitan Halifax Nova Scotia.}

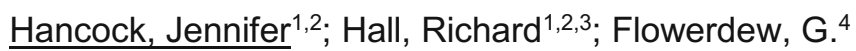

1 Faculty of Medicine, Dalhousie University, Halifax, Nova Scotia, Canada

2 Department of Critical Care, Nova Scotia Health Authority, Halifax, Nova Scotia, Canada

3 Department of Anesthesia, Pain Management \& Perioperative Medicine, Nova Scotia Health Authority, Halifax, Nova Scotia, Canada

4 Community Health \& Epidemiology Department, Dalhousie University, Halifax, Nova

Scotia, Canada

Introduction: In 2016, the Critical Care Societies Collaborative published a Call to Action in an effort to bring attention to an alarmingly high level of burnout in critical care health providers. ${ }^{1}$ Burnout syndrome develops as a result of chronically high levels of stress in the workplace and is characterized by emotional exhaustion, depersonalization and reduced personal accomplishment ${ }^{2}$. Burnout has a significant impact on healthcare providers ${ }^{3,4}$ the patients whom they care ${ }^{5,6}$ for and the system in which they work ${ }^{3,7}$.

Objective: The purpose of this survey was to identify the rates of burnout and workplace factors that may be contributing to burnout among nurses, respiratory therapists and physicians who work in the ICUs in the Department of Critical Care, Halifax, Nova Scotia.

Methods: Surveys were distributed by email link to 271 adult and pediatric ICU nurses (166), respiratory therapists (78) and physicians (27) in the Department of Critical Care, Halifax, Nova Scotia. Survey design included participant demographics, the Maslach Burnout Inventory (MBI) - Human Services Survey for Medical Personnel ${ }^{\odot}$, the Areas of Worklife Survey ${ }^{\odot}$ and a question on moral distress.

Results: 165 (60.9\%) of a possible 271 respondents participated in the survey, $88.9 \%$ of physicians, $56.0 \%$ of nurses and $61.5 \%$ of respiratory therapists. $84.2 \%$ were adult practioners. Symptoms of burnout were demonstrated in all three of the MBI domains. Emotional exhaustion was the highest with $61.8 \%$ of respondents scoring moderate or high. $43.0 \%$ scored moderate or high in depersonalization and $34.5 \%$ scored low in professional accomplishment. Table 1 shows MBI domain scores for all participants and Table 2 shows the scores by profession. $37.6 \%$ of respondents scored high for burnout in 1 domain, $10.9 \%$ scored high in two domains and $4.8 \%$ scored high in all three domains. Logistic regression showed that a longer duration of critical care work experience was associated with a lower level of emotional exhaustion and depersonalization. Pediatric healthcare providers had a lower rate of emotional exhaustion compared to adults, but no difference in depersonalization or professional accomplishment. There was no statistic difference between academic vs community centers in any domain. Analysis of the Areas of Worklife Survey showed a very strong association between five of the six subscales (workload, control, reward, fairness and values) and two of the three burnout domains (emotional exhaustion and depersonalization). $55.2 \%$ of participants reported moral distress at least a few times a month and $29.9 \%$ reported it at least once a week.

Conclusions: Burnout and moral distress is a significant issue for nurses, respiratory therapists and physicians who work in the ICUs in the Department of Critical Care, Halifax, Nova Scotia. This knowledge will help provide direction for management and support for our critical care healthcare providers moving forward. 
Table 1: MBI Domain Scores - All Participants \% (n)

\begin{tabular}{|l|c|c|c|}
\hline & Low & Moderate & High \\
\hline Emotional Exhaustion & $38.1(63)$ & $36.4(60)$ & $25.5(42)$ \\
\hline Depersonalization & $57.0(94)$ & $29.1(48)$ & $13.9(23)$ \\
\hline $\begin{array}{l}\text { Professional } \\
\text { Accomplishment }\end{array}$ & $34.5(57)$ & $31.5(52)$ & $33.9(56)$ \\
\hline
\end{tabular}

Table 2: MBI Domain Scores by Profession \% (n)

\begin{tabular}{|c|c|c|c|}
\hline & Physicians & Nurses & RTs \\
\hline Emotional Exhaustion & & & \\
Low & $54.2(13)$ & $38.7(36)$ & $29.2(14)$ \\
Moderate & $25.0(6)$ & $35.5(33)$ & $43.8(21)$ \\
High & $20.8(5)$ & $25.8(24)$ & $27.1(13)$ \\
\hline Depersonalization & $45.8(11)$ & $52.7(49)$ & $70.8(34)$ \\
Low & $37.5(9)$ & $32.3(30)$ & $18.8(9)$ \\
Moderate & $16.7(4)$ & $15.1(14)$ & $10.4(5)$ \\
High & $16.7(4)$ & $38.7(36)$ & $35.4(17)$ \\
\hline Professional & $29.2(7)$ & $30.1(28)$ & $35.4(17)$ \\
Accomplishment & $54.2(13)$ & $31.2(29)$ & $29.2(14)$ \\
Low & & & \\
Moderate & &
\end{tabular}

\section{References}

1. Moss M, Good VS, Gozal D et al. A Critical Care Societies Collaborative Statement: Burnout Syndrome in Critical Care Healthcare Professionals. Am J Respir Crit Care Med 2016; 194:106-113;

2. Maslach C, Jackson SE. MBI: Maslach burnout inventory; manual research edition. Palo Alto, CA: Consulting Psychologists Press; 1986.

3. Shoorideh FA, Ashktorab T, Yaghmaei F et al. Relationship between ICU nurses' moral distress with burnout and anticipated turnover. Nurs Ethics 2015;22:64-76.

4. Ackerman AD. Retention of critical care staff. Crit Care Med 1993; 21(9 Suppl):S394-S395.

5. Poghosyan L, Clarke SP, Finlayson M et al. Nurse burnout and quality of care: cross-national investigation in six countries. Res Nurs Health 2010;33:288-298.

6. Shanafelt TD, Balch CM, Bechamps $\mathrm{G}$ et al. Burnout and medical errors among American surgeons. Ann Surg 2010;251:995-1000.

7. Waldman JD, Kelly F, Arora S et al. The shocking cost of turnover in health care. Health Care Manage Rev 2004;29:2-7. 
A Systematic Review of the Efficacy of Mannitol Versus Hypertonic Saline in the Treatment of Raised Intracranial Pressure in Adults Suffering from Traumatic Brain Injury

$\underline{\text { Kutsogiannis, Demetrios }}^{1}$; Chan, Liza²; Hewer, Tayne'; Kim, Su Hwan³; Goondi, Damanjit ${ }^{1}$

1 Department of Critical Care Medicine, Faculty of Medicine and Dentistry, University of Alberta, Edmonton, Alberta, Canada

2 School of Library and Information Sciences, The University of Alberta, Edmonton, Alberta, Canada

3 Department of Mathematical and Statistical Sciences, University of Alberta, Edmonton, Alberta, Canada

Introduction: Raised intracerebral pressure (ICP) in the setting of traumatic brain injury (TBI) is a common problem and associated with worsening neurological outcomes. Previous systematic reviews have failed to demonstrate a survival advantage of mannitol over hypertonic saline in the management of ICP in TBI patients. However, there are no published systematic reviews comparing the magnitude and temporal durability in the reduction of ICP between the various concentrations of mannitol and hypertonic saline (HTS).

Objectives: To determine the temporal reduction in ICP using mannitol, HTS and to compare the efficacy of ICP reduction between the two agents and the effects of varying volumes and concentrations.

Methods: The review followed the guidelines of Preferred Reporting Items for Systematic Reviews and Meta-Analyses (PRISMA) as the following databases were searched: PubMed (1947 to July 2016), EMBASE (1947 to July 2016), Medline, Cochrane Databases of Systematic Review and Web of Science. The key phrases used in the search were: hypertonic saline, sodium chloride, osmotic diuretic, mannitol, intracranial pressure, traumatic brain injury, head injury. Eligibility criteria for the study included: use of an osmotic diuretic, raised intracranial pressure, adults older than 16, traumatic brain injury. Few studies had a mixed patient population of TBI and subarachnoid hemorrhage. General estimating equations for correlated longitudinal data were used to model the temporal reduction in study averaged ICP, the effect of mannitol versus HTS, and volume and concentration of therapy. Adjustment was made for study methodology.

Results: 1349 records were identified using the search strategy. Eighteen randomized controlled trials and 14 cohort studies were used in the systematic review of which 7 studies (4 randomized trials and 3 cohort studies) compared mannitol with HTS and reported mean ICP values suitable for pooled GEE analysis. Univariable ICP reductions were demonstrated at 60 minutes using HTS $(p<0.0001)$ and mannitol $(p=$ 0.02 ) but not at 30 or 120 minutes for either treatment. In the multivariable model, a significant ICP reduction was demonstrated over time $(p<0.0001)$, however there was no significant difference in ICP reduction between the HTS versus the mannitol group $(p=0.92)$. Neither treatment volume, concentration nor their interaction were modifiers of the effect of either treatment. However, non-randomized studies did modify the treatment effect [Table 1] When all available data from either single treatment (HTS or mannitol) studies or comparison studies were pooled to model the temporal reduction in ICP by individual treatment type, HTS demonstrated a more significant reduction in ICP at $30(p=0.002), 60(p<0.0001)$ and $120 \mathrm{~min}(p=0.0004)$ as compared to mannitol at $30(p=0.002), 60(p=0.004)$ and $120 \mathrm{~min}(p=0.03)$. [Figures 1,2] Using individual treatment models, a significant interaction was seen between the volume and concentration of HTS indicating optimal concentrations of $\leq 10 \%$ and volumes of $\leq 250$ $\mathrm{ml}$.

Conclusion: Both mannitol and HTS are effective at reducing ICP at 30,60, and 120 minutes after bolus dosing. Although a larger ICP reduction was demonstrated using 
HTS, this was not statistically significant. A significant interaction was noted between the volume and osmotic concentration using HTS indicating that increasing volumes of low concentration HTS does not result in a greater reduction of ICP.

Table 1. Generalized Estimating Equation multivariable model of longitudinal changes in intracerebral pressure from 0 to 120 minutes after a bolus of mannitol or hypertonic saline

\begin{tabular}{|l|r|r|r|r|}
\hline Coefficients & Parameter & $\begin{array}{l}\text { Standard } \\
\text { error }\end{array}$ & \multicolumn{1}{l|}{$\begin{array}{l}\text { Wald } \\
\text { Statistic }\end{array}$} & P-value \\
\hline Intercept & 18.0867 & 10.0951 & 3.21 & 0.073 \\
\hline Time & -0.0653 & 0.0146 & 20.03 & $<0.00001$ \\
\hline Hypertonic Saline + & -0.4063 & 3.9258 & 0.01 & 0.918 \\
\hline Volume & 0.004 & 0.0584 & 0 & 0.946 \\
\hline Concentration & 15.4056 & 55.2628 & 0.08 & 0.78 \\
\hline Retrospective $¥$ & 7.7853 & 3.2271 & 5.82 & 0.016 \\
\hline Studies & & & & \\
\hline Volume $x$ & -0.0482 & 0.3642 & 0.02 & 0.895 \\
\hline Concentration interaction & & & & \\
\hline
\end{tabular}

$†$ Mannitol as reference parameter

‡ Randomized controlled trials as reference parameter

Mean ICP over time for individual studies by study types

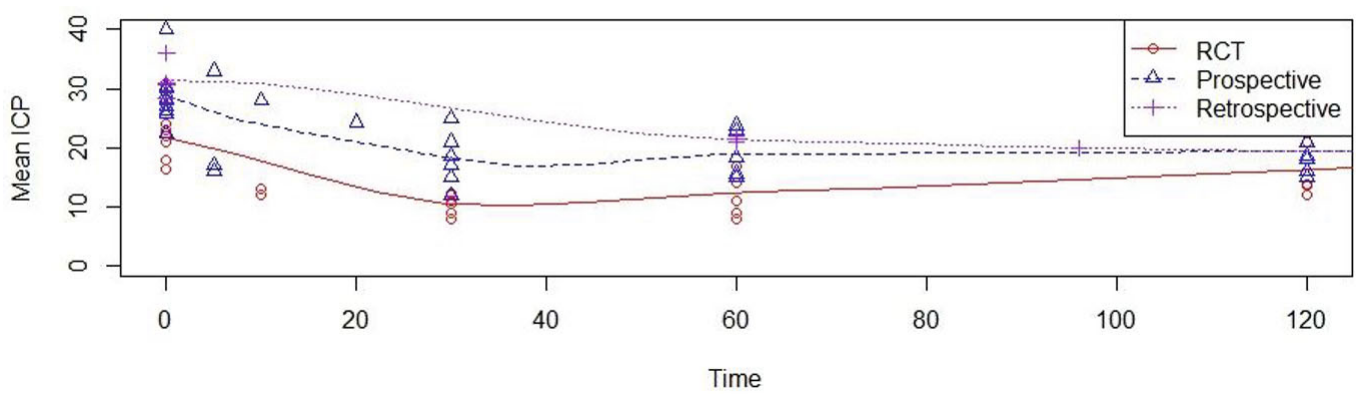

Aggregate mean ICP for all studies of each intervention (HTS,Mannitol, HES)

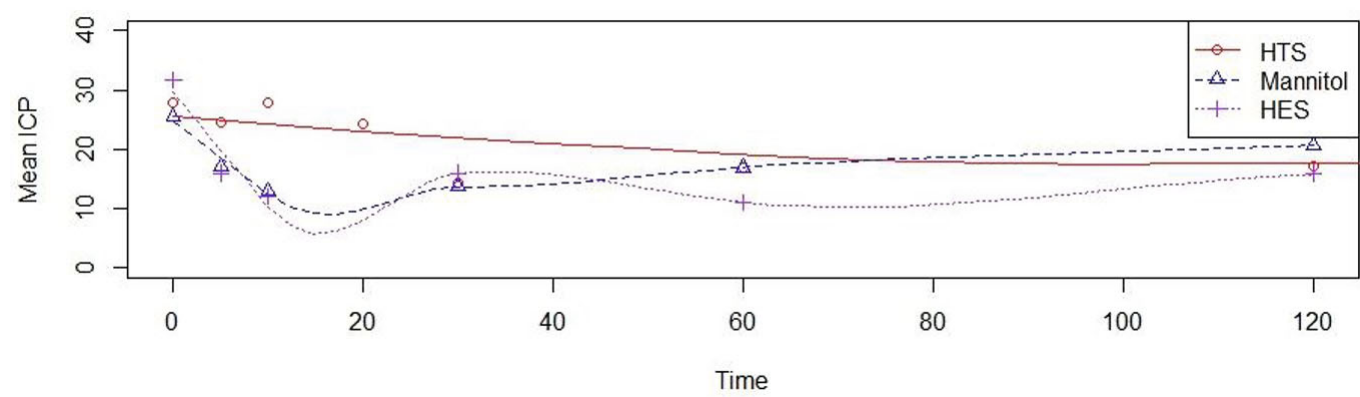




\section{References}

1. Huang X, Yang L. Comparison of $20 \%$ mannitol and $15 \%$ hypertonic saline in doses of similar osmotic burden for treatment of severe traumatic brain injury with intracranial hypertension. J South Med Univ 2014; 34 (5): 723-726

2. Alsharkasy $\mathrm{MH}$, Altouny $\mathrm{AH}$, Elshatoury HA, Ewila HA. Comparison between mannitol $20 \%$ and hypertonic saline $7.5 \%$ for cerebral resuscitation in severely head injured patients with intra-cranial hypertension. International Journal of Health Sciences 2011; 5(2): 36-37

3. Cottenceau V, Masson F, Mahamid E, Petit L, Shik V, Sztark F, Zaaroor M, Soustiel JF. Comparison of effects of equiosmolar doses of mannitol and hypertonic saline on cerebral blood flow and metabolism in traumatic brain injury. Journal of Neurotrauma 2011; 28: 2003-20012

4. Francony G, Fauvage B, Falcon D, Canet C, Dilou H, Lavagne $P$, Jacqot $C$, Payen $\mathrm{JF}$. Equimolar doses of mannitol and hypertonic saline in the treatment of increased intracranial pressure. Critical Care Medicine 2008; 36: 795-800

5. Oddo M, Levine JM, Frangos S, Carrera E, Maloney-Wilensky E, Pascual JL, Kofke WA, Mayer SA, LeRoux PD. Effect of mannitol and hypertonic saline on cerebral oxygenation in patients with severe traumatic brain injury and refractory intracranial hypertension. J Neurol Neurosurg Psychiatry 2009; 80: 916-920

6. Kerwin A, Schinco MA, Tepas JJ, Renfro WH, Vitarbo EA, Muehlberger M. The use of $23.4 \%$ hypertonic saline for the management of elevated intracranial pressure in patients with severe traumatic brain injury: A pilot study. Journal of Trauma 2009; 67 (2); 277-282

7. Ware ML, Nemani VM, Meeker M, Lee C, Morabito DJ, Manley GT. Effects of $23.4 \%$ sodium chloride solution in reducing intracranial pressure in patients with traumatic brain injury: A preliminary study. Neurosurgery 2005; 57: 727-736 
AcceleratephenoTM Potential Influence on Timeliness of Management Decisions for Patients with Positive Blood Cultures

Grant, Jennifer M. ${ }^{1}$; Porter, Chalene A. ${ }^{1}$; Charles, Marthe ${ }^{1}$; Bryce, Elizabeth A. ${ }^{1}$; Wong Titus $^{1}$; Stefanovic, Aleksandra ${ }^{1}$; Roscoe, Diane R. ${ }^{1}$

1 Department of Pathology and Laboratory Medicine, Division of microbiology, Vancouver Coastal Health, Vancouver, British Columbia, Canada

Objective: There is a direct relationship between appropriate antibiotic administration and survival in patients with sepsis encouraging broad spectrum drugs, while use of narrower spectrum antibiotics reduces adverse events and development of resistance. Rapid diagnostic testing allows more timely and accurate antibiotic prescription, thus improving clinical outcomes in patients with positive blood-cultures. The AcceleratephenoTM system (AXDX) is a platform for rapid identification (ID - 90 minutes) and susceptibility testing (AST $-<7$ hours) from positive blood culture bottles. We assessed the potential of AXDX results to influence more timely antibiotic tailoring interventions on a convenience sample of 154 positive blood cultures from unique patients.

Materials and Methods: Blood cultures with a gram stain for a single organism likely to be on the AXDX panel were run in parallel with the standard of care (SOC) when a trained technologist and machine was available for loading. Using results from the Standard of care (SOC), Medical Microbiologists noted interventions made at the time of blood culture gram stain, organism identification and AST result availability. The timing of MM intervention was noted and compared to fastest potential time in SOC and with AXDX timing.

Results: Of 154 cultures assessed by AXDX, 148 resulted in actions by either the microbiologist or medical team; $113(76 \%)$ of which were attributable to data available from AXDX. The most common AXDX attributable intervention was narrowing of antibiotics in $76(67 \%)$ cases, followed by escalation of therapy in $24(21 \%)$ instances or recommending a specialist consult (5\%). Compared to SOC, AXDX results were $15.8 \pm 12.6 \mathrm{~h}$ faster to organism ID. Likewise for AST, time-saving was $28.3 \pm 11.6 \mathrm{~h}$. Given the time saving, it would be possible to save 34 days of broad spectrum therapy by using AXDX for more timely results. For those patients requiring broader therapy, time delay to correct therapy could be decreased by $18 \mathrm{~h}$ on average.

Conclusions: There is significant potential for results from rapid diagnostic panels such as AXDX to support timely antimicrobial prescribing and other management decisions to benefit patients. Next steps include identifying septic patients in the ICU who would benefit from timelier reporting of antimicrobial susceptibility. 
Admission Frailty Laboratory Index (FI-LAB) and Associated Mortality and Costs in Older ICU Patients with Suspected Infection

Fernando, Shannon M. ${ }^{1,2}$; Mclsaac, Daniel I. 3,4,5; Perry, Jeffrey J. ${ }^{2,3,4}$; Rochwerg, Bram $^{6,7}$; Bagshaw, Sean M. ${ }^{8}$; Thavorn, Kednapa ${ }^{3,4}$; Seely, Andrew J. E. ${ }^{1,3,4,9}$; Forster, Alan J.1,3,4; Fiest, Kirsten ${ }^{10}$; Tran, Alexandre ${ }^{3,9}$; Reardon, Peter M. ${ }^{1,2}$; Tanuseputro, Peter $^{3,4,11}$; Heyland, Daren K. ${ }^{12}$; Kyeremanteng, Kwadwo ${ }^{1,4,11,13}$

1 Division of Critical Care, Department of Medicine, University of Ottawa, Ottawa, Ontario, Canada

2 Department of Emergency Medicine, University of Ottawa, Ottawa, Ontario, Canada 3 School of Epidemiology and Public Health, University of Ottawa, Ottawa, Ontario, Canada

4 Clinical Epidemiology Program, Ottawa Hospital Research Institute, Ottawa, Ontario, Canada

5 Department of Anesthesiology and Pain Medicine, University of Ottawa, Ottawa, Ontario, Canada

6 Department of Medicine, Division of Critical Care, McMaster University, Hamilton, Ontario, Canada

7 Department of Health Research Methods, Evidence, and Impact, McMaster University, Hamilton, Ontario, Canada

8 Department of Critical Care Medicine, Faculty of Medicine and Dentistry, University of Alberta, Edmonton, Alberta, Canada

9 Department of Surgery, University of Ottawa, Ottawa, Ontario, Canada

10 Department of Critical Care Medicine, University of Calgary, Calgary, AB, Canada

11 Division of Palliative Care, Department of Medicine, University of Ottawa, Ottawa, Ontario, Canada

12 Department of Critical Care Medicine, Queen's University, Kingston, Ontario, Canada

13 Institut du Savoir Montfort, Ottawa, Ontario, Canada

Introduction: Risk-stratification of patients with suspected infection is important. Existing prognostic tools (such as SIRS and qSOFA criteria) lack accuracy in older patients with suspected infection. Therefore, new methods for accurate outcome prognostication in older people with suspected infection are needed. Frailty, a syndrome based on accumulation of age- and disease-related deficits, is associated with vulnerability to adverse health outcomes. However, the association of frailty with adverse outcomes among older patients with suspected infection is poorly described. Objectives: We sought to determine the prognostic accuracy of the frailty laboratory index (FI-LAB) for prediction of in-hospital mortality among older patients (i.e. $\geq 65$ years of age) with suspected infection.

Methods: We utilized prospectively collected ICU registry data gathered from 20122016 at two hospitals in Ottawa, ON. We included consecutive patients $\geq 65$ years of age, with suspected infection (defined by the concomitant administration of oral or parenteral antibiotics, and sampling of body fluid cultures). FI-LAB was calculated using laboratory values at the time of hospital admission. To reduce the confounding influence of illness severity, we excluded patients who died within 48 hours of hospital admission, and those who presented with septic shock (as per Sepsis-3 criteria).

Patients were categorized as "Low FI-LAB" (FI-LAB <0.23), "Moderate FI-LAB" (FI-LAB $0.23-0.43)$, and "High FI-LAB" (FI-LAB >0.43). The primary outcome was in-hospital mortality, which we analyzed using a multivariable logistic regression model. We also evaluated total hospital costs.

Results: Of 1,510 included patients, 533 (35.3\%) had a Low FI-LAB, 829 (54.9\%) had a Moderate FI-LAB, and $148(9.8 \%)$ had a High FI-LAB. There was only mild correlation between FI-LAB score and illness severity, as measured by the Multiple 
Organ Dysfunction Score (MODS, $\rho=0.274$ ). Mean age was 76.3 years (SD: 7.9$)$. Inhospital mortality in the cohort was 37.0\%, 9.2\% among Low FI-LAB patients, 45.5\% among Moderate FI-LAB patients, and $89.2 \%$ among High FI-LAB patients. As compared to Low FI-LAB patients, adjusted odds of mortality among Moderate FI-LAB patients was $5.72(95 \% \mathrm{Cl}: 4.06-8.05, \mathrm{P}<0.001)$ and High FI-LAB patients was 17.15 (95\% Cl: 9.10-21.56, P < 0.001). Adjusted ORs for patient age and MODS were 1.01 (95\% Cl: 0.99-1.04, $\mathrm{P}=0.09)$ and $1.16(95 \% \mathrm{Cl}: 1.11-1.22, \mathrm{P}<0.01)$, respectively. Mean costs were significantly higher for Moderate FI-LAB patients $(\$ 47,116, \mathrm{SD}$ : $41,321)$ than Low FI-LAB patients $(\$ 42,526$, SD: 37,266$)$, and High FI-LAB patients $(\$ 37,128$, SD: 38,225) $(P<0.001)$.

Conclusions: The FI-LAB score is prognostic of in-hospital mortality among older ICU patients with suspected infection, independent of age and severity of illness. Clinicians should consider frailty (and namely, the FI-LAB) as an important prognostic factor in discussions related to treatment course and goals-of-care in this population. 
An Interprofessional and Patient-Centred Approach to Improving Transitions from Intensive Care Unit (ICU) to Ward

Austrie-Fletcher, Rina-Marie ${ }^{1} ;$ Au, Michelle²; Bitton, Marina ${ }^{3}$; Shin, Phil'; VillarGuerrero, Elizabeth ${ }^{5}$

1 Medicine/ICU, North York General Hospital, Toronto, Ontario, Canada

2 Medicine/Neuro/Stroke, North York General Hospital, Toronto, Ontario, Canada

3 Medicine/ICU, North York General Hospital, Toronto, Ontario, Canada

4 Medicine/ICU, North York General Hospital, Toronto, Ontario, Canada

5 Medicine/Neuro/Stroke, North York General Hospital, Toronto, Ontario, Canada

Introduction: Inadequately planned transfer of patients between ICU and the ward adversely impact patient/families and healthcare providers (HCPs). Patients/families report feelings of anxiety, fear, abandonment and loss of control. Similarly, HCPs on the receiving ward report increased stress due to concerns that they are unable to meet patient/family expectations and feel inadequately prepared to meet care needs. Emerging evidence suggests breakdown in the flow of information between units can lead to patient safety incidents that result in readmission to ICU and patient harm, including death. Patients may also undergo unnecessary tests and treatments leading to patient/family confusion related to perceived conflicting decision making by the health care team.

Objectives: NYGH ICU collaborated with the Medical/Stroke Unit (MSU) and the Patient and Family Advisory Committee to develop a standardized discharge protocol with the following goals:

- improve communication among multidisciplinary HCPs

- reduce medical errors associated with transitions

- reduce anxiety of patients and families during transitions of care

- improve staff and physician satisfaction

Methods: We created a standardized discharge workflow for patients with an ICU length of stay greater than 2 weeks who were discharged to the MSU. The primary outcome measure was patient/family and staff satisfaction. Secondary outcome measure was improvement of ward staff and General Internal Medicine (GIM) physicians' satisfaction with the transition process. The components of the standardized workflow included: individualized care plan with input from patient/family and multidisciplinary team including nurse, respiratory therapist, dietitian, physician, social worker; providing patients/families with Critical Care Response Team (CCRT) business card indicating they can activate a CCRT consultation; an in-person warm handoff between ICU and ward; a dictated summary of the ICU experience by intensivist. A group session was conducted to gain feedback about current transition practices from ICU to ward. A pre-survey was developed for ward staff and GIM physicians based on research articles and identified gaps with the goal of closing these gaps. Pre-implementation satisfaction surveys were distributed to multidisciplinary HCPs assuming care upon transfer from ICU. A post-implementation patient/family survey was developed based on their stories to receive feedback about transition from CrCU to ward.

Results: 26 out of 95 (27\%) ward staff completed the survey. Common themes were "unrealistic expectations" of patients/families such as nurse/patient ratio, frequency of rounding and monitoring; and inadequate information sharing to ward staff to provide care to complex patients. 9 out of $9(100 \%)$ GIM physicians completed the survey and $100 \%$ of participants were dissatisfied with the current transition process. Since implementation, 5 out of $5(100 \%)$ patients had a dictated intensivist summary, interprofessional warm hand-off, and individualized care plan. We do not have any baseline patient and family surveys; however, we are currently collecting post intervention/implementation surveys focused on transitions and overall experience. 
Conclusion: Preliminary findings indicate that this QI initiative has resulted in improved communication and standardization of the transition process. Future success measures will be achieving an increase in patient, family, staff and physician satisfaction.

\section{References}

1. Buchner, D. L., Bagshaw, S.M., Dodek, P., Forster, A. J., Fowler, R. A., Lamontage, F., Turgeon, A. F., Potestio, M, \& Stelfox, H. T. (2015). Prospective cohort study protocol to describe the transfer of patients from intensive care units to hospital wards. BMJ Open 5(7). doi: 10.1136/bmjopen-2015-007913

2. Haggstrom, M., Asplund, K., \& Kristiansen, L. (2009). Struggle with a gap between intensive care units and general wards. International Journal of Qualitative Studies on Health and Well-being, 4(3), 181-192. doi: 10.108/17482620903072508

3. Haggstrom, M., \& Backstrom, B. (2014). Organizing Safe Transitions from Intensive Care. Nursing Research and Practice, 2014. doi: 10.1155/2014/175314

4. Stelfox, H. T., Leigh, J. P., Dodek, P. M., Turgeon, A. F., Forster, A. J., Lamontagne, F., Fowler, R. A., Soo, A., \& Bagshaw, S. M. (2017). A multi-center prospective cohort study of patient transfers from the intensive care unit to the hospital ward. Intensive Care Med, 43(10), 1485-1494. doi:10.1007/s00134-017-4910-1 


\section{Cardiovascular ICU Nurses' Feedback on the Use of the CHG-Lock ${ }^{\mathrm{TM}}$ : A Survey as Part of the CHG Pilot Study}

Pook, Makena ${ }^{1}$; McDonald, Ellen ${ }^{1,2}$; Benoit, Pamela ${ }^{3}$; Coyne, Alice ${ }^{3}$; Blair, Melanie ${ }^{3}$; Zamir*, Nasim $^{1,3}$; Fox-Robichaud ${ }^{*}$, Alison ${ }^{1,3}$

1 McMaster University, Hamilton, Ontario, Canada

2 Canadian Critical Care Trials Group, Canada

3 Hamilton Health Sciences, Hamilton, Ontario, Canada

Background: CHG-Lock ${ }^{\mathrm{TM}}$ is a new medical device being piloted in a feasibility RCT trial $^{1}$. The device aims to decrease the risk of central line associated bloodstream infections (CLABSIs) in an ICU setting. CLABSIs account for $87 \%$ of all primary nosocomial bloodstream infections in North America, leading to increased mortality, length of stay, and readmissions ${ }^{2,3}$. The use of CHG-Lock ${ }^{\mathrm{TM}}$ requires Registered Nurses (RNs) to perform additional steps during routine intravenous line care in an already busy environment. It is unknown how RNs involved in the trial will operationalize the study for optimal compliance with the protocol.

Objectives: To assess uptake of the CHG-Lock ${ }^{\mathrm{TM}}$ protocol by ICU RNs working in our Cardiac Surgical Unit to determine areas of improvement for compliance before expanding the study to additional ICUs.

Methods: We developed a 9-item questionnaire under 4 domains: 1. comfort with and ease of study related tasks, 2. time for device use and documentation, 3. compliance, and 4. effectiveness of educational activities and support material. Respondents also provided demographic data including years of overall nursing experience and experience in the CV-ICU. Prior to distribution, the questionnaire was pilot tested using a convenience sample of 6 ICU RNs. Further refinement of the questionnaire involved clinical sensibility testing using a convenience sample of 6 ICU RNs who provided feedback on appropriateness, redundancy and completion time of the tool. To provide RNs with reasonable learning time and exposure to CHG-Lock ${ }^{\mathrm{TM}}$, we waited until 10 patients had completed the study before we began questionnaire administration. Paper versions of the questionnaire were distributed to RNs that cared for study patients. Results: Surveys were completed by 22 of $38(57 \%)$ RNs, caring for at least one of 6 study patients. Twelve $(60 \%)$ nurses reported greater than 15 years nursing experience and $11(55 \%)$ had greater than 15 years CV-ICU experience. All RNs found using the device to be either "easy", "very easy" [18(85.7\%)] or "not difficult" [3(14.3\%)]. Most nurses $13(65 \%)$ ) "felt comfortable using the device", 11(55\%) reported less than 1 minute required for use of the device and $12(60 \%)$ reported less than 1 minute to document each use. Two thirds of RNs found using the device presented 'no change' to their workload [14(66.7\%)]. The most common reasons for failing to use CHGLock $^{\mathrm{TM}}$ properly (i.e. withdraw from line or lock as indicated) were: $6(33.3 \%)$ required urgent access to IV; $4(22.2 \%)$ forgot; and $3(16.7 \%)$ were unaware patient was in the study. Although less than $80 \%$ of RNs found educational activities and support material "effective", there was room for improvement. Open ended responses revealed the need for a shorter interval between in-servicing and patient enrollment. Respondents who found study staff more helpful also reported CHG was easier to use $(\mathrm{d}=0.439$, $p=0.047)$, required less time for use $(d=-0.576, p<0.001)$, and increased their workload less $(\mathrm{d}=0.351, \mathrm{p}=0.019)$.

Conclusions: In this pilot study for a new device to reduce bacteremia in an ICU patient population, RNs reported use of the device was not time-consuming or burdensome. There is room for improvement of educational activities and support material. We have created a video outlining RN's responsibilities, which should address this limitation. In-servicing should occur in tandem with patient enrollment. We will engage Nurse Champions to provide RN support. Moving forward, we will pilot and evaluate these new educational initiatives. 


\section{References}

1. Kowalewska PM, Petrik SM, Di Fiore, AE and Fox-Robichaud AE. Antimicrobial efficacy of a new chlorhexidine-based device against Staphylococcus aureus colonization of venous catheters. J Infusion Nursing 2018 April; 41(2):103-112.

2. Ziegler MJ, Pellegrini DC, Safdar N. Attributable mortality of central line associated bloodstream infection: systematic review and meta-analysis. Infection. 2015 Feb;43(1):29-36.

3. Richard MJ, Edwards JR, Culver DH, Gaynes RP. Nosocomial infections in medical intensive care units in the United States. National Nosocomial Infections Surveillance System. Crit Care Med. 1999 May;27(5):887-892. 
CHG-Lock Device for Prevention of Central line Infections in the ICU - An Open Label Randomized Pilot Study: Protocol

Zamir, Nasim¹; McDonald, Ellen²; Jackson Chornenki, Nicholas³; Fox-Robichaud, Alison ${ }^{4}$

1 College of Medicine, University of Saskatchewan, Saskatoon, SK, Canada

2 National Platform Research Coordinator, Critical Care Canada Trials Group and Canadian Critical Care Research Coordinators Group, Canada

3 Michael G. DeGroote School of Medicine, McMaster University, Hamilton, Ontario, Canada

4 Department of Medicine, Division of Critical Care, McMaster University, Hamilton, Ontario, Canada

Background: In North America, $87 \%$ of nosocomial primary bloodstream infections are attributed to central venous catheters (CVCs). ${ }^{1}$ Infection rates from CLABSI have halved between 2006 and 2011 due to basic prevention practices, optimized training, technology and evidence application. ${ }^{2,3}$ However, touch contamination of catheter hubs continues to pose a prominent mode of infection. Chlorhexidine Gluconate (CHG) is a broad-spectrum biocide with a wide range of antimicrobial activity. ${ }^{4}$ Catheters impregnated with CHG have shown to significantly reduce CLABSIs and catheter colonization in the ICU. ${ }^{5}$ These catheters are expensive and have a short halflife. ${ }^{6}$ The CHG-Lock ${ }^{\mathrm{TM}}$ device, is a sterile attachment loaded with freeze-dried CHG that luerlocks onto a prefilled syringe, sterilizing the solution and line when solutions are passed through. This is a potential therapy combat touch contamination of CVC.

Objective: The aim of the CHG-Lock ${ }^{\mathrm{TM}}$ pilot trial (NCT03309137) is to determine the feasibility of conducting a larger trial of CHG-Lock TM devices to reduce CLABSI in the intensive care unit (ICU). Secondary objective is to measure a signal for benefit in reducing morbidity and mortality secondary to CLABSI.

Methods: Adults admitted to the ICU expected to remain for $\geq 72$ hours with a central line in-situ will be randomized to the CHG-Lock ${ }^{\mathrm{TM}}$ device or standard of care. We will exclude patients with a chronic indwelling catheter, suspected infection, or CHG allergy. Randomization will be in variable block sizes at a fixed 1:1 ratio. To ensure allocation concealment, a third party will prepare sequentially numbered, opaque, sealed envelopes. ${ }^{7}$ Patients randomized to the CHG-Lock ${ }^{\mathrm{TM}}$ arm will have all central and peripheral IV lines locked with the device and unused ports relocked every 24 hours or after use. Blood cultures will be collected from all patients with a central line every 48 hours until central line removal or ICU transfer.

Results: Feasibility will be determined by: 1) $\geq 80 \%$ consent; 2) Recruitment of 100 patients over 1 year; and 3) Protocol adherence; and 3) Level of comfort with the protocol. The latter two will be assessed through anonymized ICU nurse surveys with continuous improvements to the protocol as needed. The clinical outcomes to be measured include: CVC colonization, CLABSI, mortality, and length of stay. Analysis will be conducted with the intention to treat principle. Kaplan-Meier curves will demonstrate proportion of CVCs remaining free of microbial colonization. Cox regression to estimate HRs. NNTB to avoid colonization and to avoid CLABSI. Comparison of rates of colonization and CLABSI will be expressed as number of events reported per 1000 CVC days. Metrics such as mean, median and mode will be used for descriptive analysis.

Discussion: Touch contamination of central lines and hematogenous spread secondary to enteric bacterial translocation are common causes of CLABSI in the critically ill. We present our protocol to encourage investigators to consider that device trials, like drug trials, require rigorous assessment. Compared to CHG impregnated catheters, the CHG-Lock ${ }^{\mathrm{TM}}$ has the potential of wider and cost-effective clinical applicability in the context of infection prevention. The recruitment of 100 patients will 
allow us to determine an appropriate sample size with signal for benefit for the full trial.

\section{References}

1. Richards, M. J., et al. (2000). "Nosocomial infections in combined medical-surgical intensive care units in the United States." Infect Control Hosp Epidemiol 21(8): 510515.

2. Public Health Agency of Canada. http://publications.gc.ca/pub?id=9.698745\&sl=0.

3. Fowler, R. A., et al. (2015) "Critical care capacity in Canada: results of a national cross-sectional study." Crit Care 19: 133.

4. Kowalewska, P. M., et al. (2018). "Antimicrobial efficacy of a new chlorhexidinebased device against Staphylococcus aureus colonization of venous catheters." J Infus Nurs 41(2): 103-112.

5. Wassil, S. K., et al. (2007). "Antimicrobial impregnated catheters in the prevention of catheter-related bloodstream infection in hospitalized patients." J Pediatr Pharmacol Ther 12(2): 77-90.

6. Neugebauer, E. A. M., et al. (2017). "Specific barriers to the conduct of randomised clinical trials on medical devices." Trials 18(1): 427.

7. Forder, P. M., Gebski, V. J., \& Keech, A. C. (2005). Allocation concealment and blinding: when ignorance is bliss. Med J Aust, 182(2), 87-89. 


\section{Comparison of Outcomes and Costs Between Adult Diabetic Ketoacidosis} Patients Admitted to the ICU or Stepdown Unit

Fernando, Shannon M. ${ }^{1,2}$; Bagshaw, Sean M. ${ }^{3}$; Rochwerg, Bram ${ }^{4,5}$; Mclsaac, Daniel|'.6,7,8; Thavorn, Kednapa $^{7,8}$; Forster, Alan J. ${ }^{1,7,8}$; Meggison, Hilary ${ }^{1}$; Tran, Alexandre ${ }^{7,9}$; Reardon, Peter M. ${ }^{1,2}$; Rosenberg, Erin ${ }^{1}$; Tanuseputro, Peter ${ }^{7,8,10}$; Kyeremanteng, Kwadwo ${ }^{1,8,10,11}$

1 Division of Critical Care, Department of Medicine, University of Ottawa, Ottawa, ON 2 Department of Emergency Medicine, University of Ottawa, Ottawa, Ontario, Canada 3 Department of Critical Care Medicine, Faculty of Medicine and Dentistry, University of Alberta, Edmonton, Alberta, Canada

4 Department of Medicine, Division of Critical Care, McMaster University, Hamilton, Ontario, Canada

5 Department of Health Research Methods, Evidence, and Impact, McMaster University, Hamilton, Ontario, Canada

6 Department of Anesthesiology and Pain Medicine, University of Ottawa, Ottawa, Ontario, Canada

7 School of Epidemiology and Public Health, University of Ottawa, Ottawa, Ontario, Canada

8 Clinical Epidemiology Program, Ottawa Hospital Research Institute, Ottawa, Ontario, Canada

9 Department of Surgery, University of Ottawa, Ottawa, Ontario, Canada

10 Division of Palliative Care, Department of Medicine, University of Ottawa, Ottawa,

Ontario, Canada

11 Institut du Savoir Montfort, Ottawa, Ontario, Canada

Introduction: There is wide variation in the utilization of Intensive Care Unit (ICU) beds for treatment and monitoring of adult patients with Diabetic Ketoacidosis (DKA). In some centres, all DKA patients are admitted to the ICU, while in others, these patients are admitted to step-down units, where close monitoring can still be provided. Given the limited availability of ICU beds, the effectiveness of such step-down units must be evaluated.

Objectives: We sought to compare the outcomes and costs of adult DKA patients admitted to an ICU against those admitted to a step-down unit.

Methods: We utilized prospectively collected registry data gathered from 2011-2016 at two hospitals in Ottawa, ON. We included consecutive patients admitted to hospital, and who met diagnostic criteria for DKA (as defined by the American Diabetes Association). Patients were either admitted to the ICU, or a step-down unit, which has a nurse-to-patient ratio of 2:1, but does not have capability for mechanical ventilation or administration of vasoactive agents. Patients admitted to ICU for reasons other than DKA were excluded. Patients requiring treatment that could not be provided in the stepdown unit (e.g. mechanical ventilation, vasoactive agents) were similarly excluded. The primary outcome was in-hospital mortality, analyzed using a multivariable logistic regression model, adjusting for age, comorbidities, and illness severity. We secondarily evaluated total hospital costs, with variation in total costs assessed using a multivariable generalized linear model with log link. A gamma distribution was applied.

Results: 872 patients were included in analysis, of which $71(8.1 \%)$ were admitted to ICU, while the remaining 801 (91.9\%) were admitted to a stepdown unit. In both groups, patients predominantly had Type 1 Diabetes Mellitus $(80.5 \%$ in ICU vs. $78.7 \%$ in step-down, $\mathrm{P}=0.70)$, and no differences in chronic complications from diabetes were found. There was no difference in median venous $\mathrm{pH}$ at admission (7.07 in ICU vs. 7.10 in step-down, $P=0.17$ ). The most common precipitant for DKA in both cohorts was medication non-compliance ( $83.1 \%$ in ICU vs. $86.2 \%$ in step-down, $P=0.41) .65$ 
total patients died in-hospital (7.5\%). No difference in in-hospital mortality was found between patients admitted to the ICU and those admitted to the step-down unit (adjusted OR: 1.14, 95\% Cl: 0.87-2.64). Mean total costs were significantly higher for patients admitted to the ICU $(\$ 20,428$ vs. $\$ 6,484, \mathrm{P}<0.001)$. Admission to ICU had an independent effect on total costs in the generalized linear model (coefficient 2.26, $\mathrm{P}<$ 0.001).

Conclusions: Adult DKA patients admitted to a step-down unit had comparable inhospital mortality and lower overall costs than those admitted to the ICU. Individual hospitals should consider such step-down units in order to reduce resource utilization and overall costs. 
Comparison of The Source and Prognostic Utility of Cfdna In Trauma and Sepsis

Jackson Chornenki, Nicholas L. ${ }^{1,2}$; Coke, Rob ${ }^{1}$; Kwong, Andrew C. ${ }^{2}$; Dwivedi, Dhruva J.2; McDonald, Ellen²; Marshall, John C. ${ }^{3}$; Fox-Robichaud, Allison E. ${ }^{1,2}$; Charbonney, Emmanuel4; Liaw, Patricia C. ${ }^{1,2}$

1 Department of Medicine, McMaster University, Hamilton, Ontario, Canada

2 Thrombosis and Atherosclerosis Research Institute, Hamilton, Ontario, Canada

3 St. Michaels Hospital, Toronto, Ontario, Canada

4 The Montreal Sacred Heart Hospital Research Center, Montreal, Quebec, Canada

Introduction: Traumatic injuries are a major cause of morbidity and mortality worldwide. In trauma patients, cell and tissue death can result from inflammation or injury. Cell-free DNA (cfDNA) has anti-fibrinolytic and pro-coagulant effects and can be released by neutrophils via NETosis in response to infection, sterile inflammation, and hypoxia or by processes like necrosis. Previously, cfDNA has been noted to be elevated and prognostic in sepsis. In patients with severe traumatic brain injury, high levels of cfDNA were associated with an increased risk of death. Protein $C$ (PC) is a natural anticoagulant that inhibits coagulation in the microcirculation. Low PC levels are associated with increased risk of death in sepsis.

Objective: to determine if cfDNA or PC, previously shown to be capable of discriminating between survivors and non-survivors in sepsis, had prognostic utility in trauma patients. Methods: Patient plasma samples were obtained from a combination of two prospective observational cohort studies: DYNAMICS (NCT0135504) and ENPOLY. Clinical data and plasma samples were obtained on admission, daily for the first week, and then weekly thereafter. Levels of cfDNA, PC, and Myeloperoxidase (MPO) were measured in the plasma using established laboratory protocols. Using 293XL-hTLR9 reporter cells, we determined the levels of NF-kB activation by these cells in response to cfDNA from the ENPOLY samples at all time points.

Results: A total of 77 trauma patients were included $(n=38$ from DYNAMICS and $n=39$ from ENPOLY). The median age was 49 years; $27.3 \%$ were female. Mortality was $16.9 \%$ at 28 days. Levels of cfDNA were elevated compared to healthy values in our patient population, but not significantly different between survivors and non- survivors. PC levels were reduced in our population compared to normal levels but did not differ significantly between survivors and non-survivors. Platelet count on admission was significantly lower in non-survivors than survivors $(p<0.01)$ suggesting an underlying consumptive process. Receiver Operating Characteristic curves for predicting mortality are shown in Table 1. When plasma samples were added with 293XL-hTLR9 reporter cells, there was no production of SEAP at any time point, indicating no NF-kB activation and the cfDNA in trauma samples is not bacterial in origin. When MPO levels were quantified as a surrogate marker of NETosis, there was no correlation to cfDNA levels $(r=-0.192, p=0.115)$; however, a positive correlation existed between MPO and cfDNA in a comparison group of septic patients $(n=52)$ from the DYNAMICS study $(r=0.424, p<0.001)$.

Conclusion: Levels of cfDNA and PC are abnormal in multiple critical conditions such as sepsis and trauma. However, variation in the source of cfDNA between septic and trauma patients may account for differences in prognostic utility. In trauma patients, future studies into dysregulation of platelet production and function may prove useful for improving survival. 


\section{Complications of Computed Tomographic Pulmonary Angiography to Rule Out Pulmonary Embolism in a Tertiary Care Cancer \& Neuroscience Center}

Alabdrab Alnabi, Mohammed ${ }^{1}$; AlGhamdi, Mohammed ${ }^{2}$; AlShammasi, Heba ${ }^{3} ;$ Al Bader, Suha2; Osman, Ibrahim²; AlAbyad, Lina ${ }^{4}$; AlHayki, Alaa ${ }^{5}$; Talaq, Faiza ${ }^{2}$

1 Maternity \& Children Hospital, Dammam, Saudi Arabia

2 King Fahad Specialist Hospital, Dammam, Saudi Arabia

3 Dammam Medical Complex, Dammam, Saudi Arabia

4 Murjan Medical Complex, Dammam, Saudi Arabia

5 Imam Abdulrahman Bin Faisal University, Dammam, Saudi Arabia

Background: Pulmonary embolism is a difficult diagnosis, with potentially serious consequences, but computed tomographic pulmonary angiography is also not without complications. Our hospital is a cancer and neuroscience center with high risk for pulmonary embolism, and high frequency of performing computed tomographies for it. Methods: This is a prospective observational study designed to measure all the potential complications of computed tomographic pulmonary angiographies in adults (including contrast-induced nephropathy and allergic reactions, as well as lifetime radiation related risk of cancer and cancer mortality). We then compared this to the incidence of pulmonary embolism and the frequency of its complications in the same cohort (mechanical ventilation, use of vasopressors, and hemorrhage from thrombolysis), as well as the pulmonary embolism cases that could have been missed if computed tomography was only performed for high risk patients.

Results: We analyzed a sample of 134 consecutive patients who presented to the emergency department between March 2014 and December 2017. We found a 13.4\% incidence of contrast-induced nephropathy (18/134 patients). None of them required dialysis \& nobody developed allergic reactions. Lifetime risk of cancer attributable to this computed tomography exposure was estimated to increase by $0.147 \%$ \& risk of death by $0.071 \%$. The incidence of confirmed pulmonary embolism in the same sample was $20.1 \%$ (27/134 patients), 3 of them died because of pulmonary embolism (representing $11.1 \%$ from patients with confirmed pulmonary embolism). $11.2 \%$ required mechanical ventilation (15/134 patients), and 5.2\% required vasopressors (7/134 patients). Only 1 patient re-quired thrombolytic therapy $(0.7 \%)$. He did not develop hemorrhage after treatment. Compared to the $11.1 \%$ mortality rate of treated cases, the historical mortality rate of undiagnosed and untreated cases has been reported between $26-30 \%$. If computed tomography was restricted to high clinical risk cases, we could have missed $55 \%$ of pulmonary embolism cases, resulting in a potential mortality increase from $11.1 \%$ to $20.2 \%$.

Conclusion: The incidence of pulmonary embolism and its complications in this patient population was higher and more serious than the incidence of computed tomography complications. Therefore, we would still recommend maintaining a low threshold of obtaining a computed tomography pulmonary angiography in this patient population.

\section{Table 1 - Primary Endpoints}

\begin{tabular}{|l|l|}
\hline Endpoint & MEAN (\%) \\
\hline Patients with Contrast Induced Nephropathy & $18(13.4 \%)$ \\
\hline Patients requiring dialysis & $0(0 \%)$ \\
\hline Patients with Renal Failure Mortality & $0(0 \%)$ \\
\hline Patients with contrast Allergy & $0(0 \%)$ \\
\hline $\begin{array}{l}\text { Patients with Pulmonary Embolism } \\
\text { Patients with Pulmonary Embolism Mortality }\end{array}$ & $27(20.1 \%)$ \\
\hline
\end{tabular}


Table 2 - Lifetime Cancer, Leukemia, \& Mortality Risks Attributable to Exposure to $1 \mathrm{CTPA}$

\begin{tabular}{|c|c|c|c|c|c|c|c|}
\hline & \multicolumn{2}{|c|}{ Males $(\# / \mathbf{1 0 0 , 0 0 0 )}$} & \multirow{2}{*}{$\begin{array}{c}\text { Males (\%) } \\
\text { Absolute } \\
\text { Risk } \\
\text { Increase (\%) }\end{array}$} & \multicolumn{2}{|c|}{ Females $(\# / \mathbf{1 0 0 , 0 0 0 )}$} & \multirow{2}{*}{$\begin{array}{c}\text { Females (\%) } \\
\text { Absolute } \\
\text { Risk } \\
\text { Increase (\%) }\end{array}$} & \multirow{2}{*}{$\begin{array}{c}\text { Both } \\
\text { Genders } \\
\text { Absolute } \\
\text { Risk } \\
\text { Increase (\%) }\end{array}$} \\
\hline & $\begin{array}{c}\text { Baseline } \\
\text { Risk }\end{array}$ & $\begin{array}{c}\text { Absolute } \\
\text { Risk } \\
\text { Increase (\#) }\end{array}$ & & $\begin{array}{l}\text { Baseline } \\
\text { Risk }\end{array}$ & $\begin{array}{c}\text { Absolute } \\
\text { Risk } \\
\text { Increase (\#) }\end{array}$ & & \\
\hline Lifetime Cancer Risk & 45500 & 56 & $0.056 \%$ & 36900 & 91 & $0.091 \%$ & $0.147 \%$ \\
\hline $\begin{array}{l}\text { Lifetime Cancer } \\
\text { Deaths }\end{array}$ & 22100 & 28.7 & $0.029 \%$ & 17500 & 42.7 & $0.043 \%$ & $0.071 \%$ \\
\hline $\begin{array}{l}\text { Lifetime Leukemia } \\
\text { Risk }\end{array}$ & 830 & 7 & $0.007 \%$ & 590 & 4.9 & $0.005 \%$ & $0.012 \%$ \\
\hline $\begin{array}{l}\text { Lifetime Leukemia } \\
\text { Deaths }\end{array}$ & 710 & 4.2 & $0.004 \%$ & 530 & 3.5 & $0.004 \%$ & $0.008 \%$ \\
\hline
\end{tabular}

Figure 1

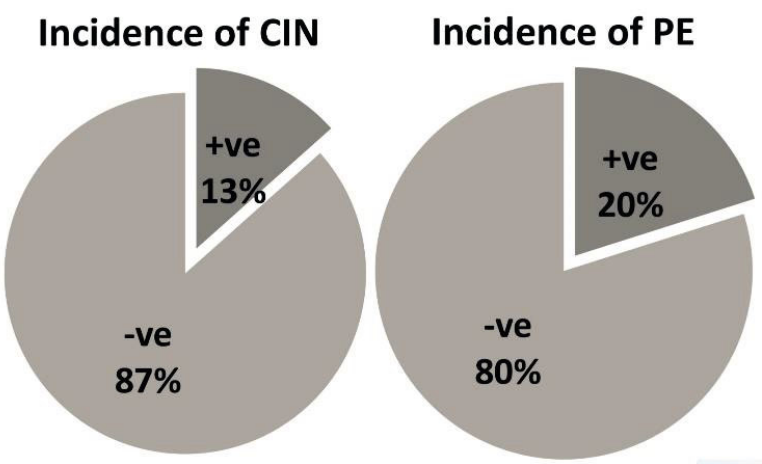

\section{References}

1. Torbicki A, Perrier A, Konstantinides S, et al. Guidelines on the diagnosis and management of acute pulmonary embolism: the Task Force for the Diagnosis and Management of Acute Pulmonary Embolism of the European Society of Cardiology (ESC). Eur Heart J. 2008; 29:2276-315.

2. Courtney DM, Sasser H, Pincus B, et al. Pulseless electrical activity with witnessed arrest as a predic-tor of sudden death from massive pulmonary embolism in outpatients. Resuscitation. 2001; 49:265-72.

3. Lessler AL, Isserman JA, Agarwal R, et al. Testing low-risk patients for suspected pulmonary embolism: a decision analysis. Ann Emerg Med. 2010; 55:316-26.

4. Kline JA, Courtney DM, Beam DM, et al. Incidence and predictors of repeated computed tomographic pulmonary angiography in emergency department patients. Ann Emerg Med. 2009; 54:41-8.

5. Brenner DJ, Hall EJ. Computed tomography-an increasing source of radiation exposure. N Engl J Med. 2007; 357:2277-84.

6. Einstein AJ, Henzlova MJ, Rajagopalan S. Estimating risk of cancer associated with radiation exposure from 64-slice computed tomography coronary angiography. JAMA. 2007; 298:317-23.

7. Kooiman J, Klok FA, Mos IC, et al. Incidence and predictors of contrast-induced nephropathy follow-ing CT-angiography for clinically suspected acute pulmonary embolism. J Thromb Haemost. 2010; 8:409-11. 
8. Mitchell AM, Kline JA. Contrast nephropathy following computed tomography angiography of the chest for pulmonary embolism in the emergency department. $\mathrm{J}$ Thromb Haemost. 2007; 5:50-4.

9. Mitchell AM et al. Prospective Study of the Incidence of Contrast-induced Nephropathy Among Patients Evaluated for Pulmonary Embolism by Contrast enhanced Computed Tomography. Acad Emerg Med June 2012;19:618-625.

10. Barrett BJ, Katzberg RW, Thomsen HS, et al. Contrast-induced nephropathy in patients with chronic kidney disease undergoing computed tomography: a doubleblind comparison of iodixanol and iopamidol. Invest Radiol. 2006; 41:815-21.

11. Harjai KJ, Raizada A, Shenoy C, et al. A comparison of contemporary definitions of contrast nephropathy in patients undergoing percutaneous coronary intervention and a proposal for a novel nephrop- athy grading system. Am J Cardiol. 2008; 101: 812-9.

12. Solomon RJ, Mehran R, Natarajan MK, et al. Contrast-induced nephropathy and long-term adverse events: cause and effect? Clin J Am Soc Nephrol. 2009; 4:1162-9.

13. Gami AS, Garovic VD. Contrast nephropathy after coronary angiography. Mayo Clin Proc. 2004; 79:211-9.

14. Kline JA, Mitchell AM, Runyon MS, et al. Electronic medical record review as a surrogate to telephone follow-up to establish outcome for diagnostic research studies in the emergency department. Acad Emerg Med. 2005; 12:1127-33.

15. Hou SH, Bushinsky DA, Wish JB, et al. Hospital-acquired renal insufficiency: a prospective study. Am J Med. 1983; 74:243-8.

16. Walter Huda, Radiation Doses and Risks in Chest Computed Tomography Examinations. Proc Am Thorac Soc Vol 4. pp 316-320, 2007.

17. Pim A. de Jong, John R. Mayo, Kamran Golmohammadi, Yasutaka Nakano, Maarten H. Lequin, Harm A. W. M. Tiddens, John Aldrich, Harvey O. Coxson, and Don D. Sin. Estimation of Cancer Mortality Associated with Repetitive Computed Tomography Scanning. Am J Respir Crit Care Med Vol 173. pp 199-203, 2006

18. Wells PS, Anderson DR, Rodger M, et al. Derivation of a simple clinical model to categorize patients' probability of pulmonary embolism: increasing the model's utility with the SimpliRED D-dimer. Thromb Haemost. 2000;83:416-420.

19. Van Belle A, Buller HR, Huisman MV, et al for the Christopher Study Investigators. Effectiveness of managing suspected pulmonary embolism using an algorithm combining clinical probability, D- dimer testing, and computed tomography. JAMA 2006;295:172-179. 


\section{Determining the Optimal Time for LibEration from Renal Replacement Therapy in} Critically III Patients: A Systematic Review and Meta-Analysis (DOnE RRT)

Katulka, Riley; Al Saadon, Abdalrhman; Sebanstianski, Meghan²; Featherston, Robin ${ }^{2,3}$; Vandermeer, Ben ${ }^{3}$; Gibney, Noel ${ }^{1}$; Rewa, Oleksa ${ }^{1}$; Bagshaw, Sean ${ }^{1}$ 1 Department of Critical Care Medicine, Faculty of Medicine and Dentistry, University of Alberta, Edmonton, Alberta, Canada

2 Alberta Strategy for Patient Oriented Research (SPOR) SUPPORT Unit Knowledge

Translation Platform, University of Alberta, Edmonton, Alberta, Canada

3 Alberta Research Center for Health Evidence (ARCHE), University of Alberta, Edmonton, Alberta, Canada

Introduction: Renal replacement therapy (RRT) is a complex and expensive form of life-sustaining therapy, reserved for our most acutely ill patients. While a number of randomized trials have evaluated the optimal timing to start RRT among critically ill patients in the ICU, there has been a paucity of trials providing guidance on when and under what circumstances to ideally liberate a patient from RRT.1,2,3

Objectives: To conduct a systematic review and meta-analysis of available evidence on clinical and biochemical markers that predict renal recovery and successful liberation from acute RRT among critically ill patients with acute kidney injury (AKI).

Methods: A comprehensive search strategy was developed in consultation with a research librarian and independently peer-reviewed by a second librarian. Electronic databases (Ovid Medline, Ovid Embase and Wiley Cochrane Library) were searched, as were selected grey literature sources. Our search strategy focused on concepts related to RRT (i.e., intermittent hemodialysis (IHD), slow low-efficiency dialysis (SLED), continuous renal replacement therapy (CRRT)); intensive care medicine (i.e., involving any intensive care unit (ICU) setting) and discontinuation of therapy (i.e., either clinical, physiological and biochemical parameters of weaning acute RRT). Citation screening, selection, quality assessment and data abstraction were performed in duplicate. Studies were pooled in statistical meta-analysis when deemed sufficiently clinically homogenous, with sensitivities and specificities pooled simultaneously using a hierarchical summary receiver operator characteristic curve (HSROC) and bi-variate analysis.

Results: Our search yielded 3031 results, of which 20 studies fulfilled eligibility; 18 cohort studies and 2 case control studies, evaluating a total of 3650 patients (Figure 1). Studies were assessed for quality and risk of bias using the Newcastle-Ottawa Score (NOS). The mean NOS score was 7 (range 4-9) and the majority of studies were of high quality ( $n=16,80 \%)$ with 4 studies rated as moderate quality $(20 \%)$. There were no studies identified as being poor quality. The most commonly identified parameter was urine output, identified in 9 studies $(45 \%)$, followed by serum Cystatin C, which was identified in 3 studies (15\%). Other identified biomarkers included kinetic eGFR, plasma NGAL, urine urea, creatinine clearance (2- and 24- hour), NT-proBNP and multivariate models including baseline parameters, each identified in a single instance. However, all identified parameters exhibited significant heterogeneity between studies. Conclusion: 20 studies were identified evaluating clinical and biochemical parameters of weaning RRT in critically ill patients with AKI. Urine output and serum cystatin C were the most commonly identified parameters, although definitions and cutoff values were heterogenous between studies. Further study is required to better define cutoffs and clarify the role of various biomarkers and elucidate accurate predictive models of RRT weaning in the critically ill patient population. Future work will involve quantitative analysis of the various identified weaning parameters. 


\section{Figure 1 - Flow Diagram of Study Selection}

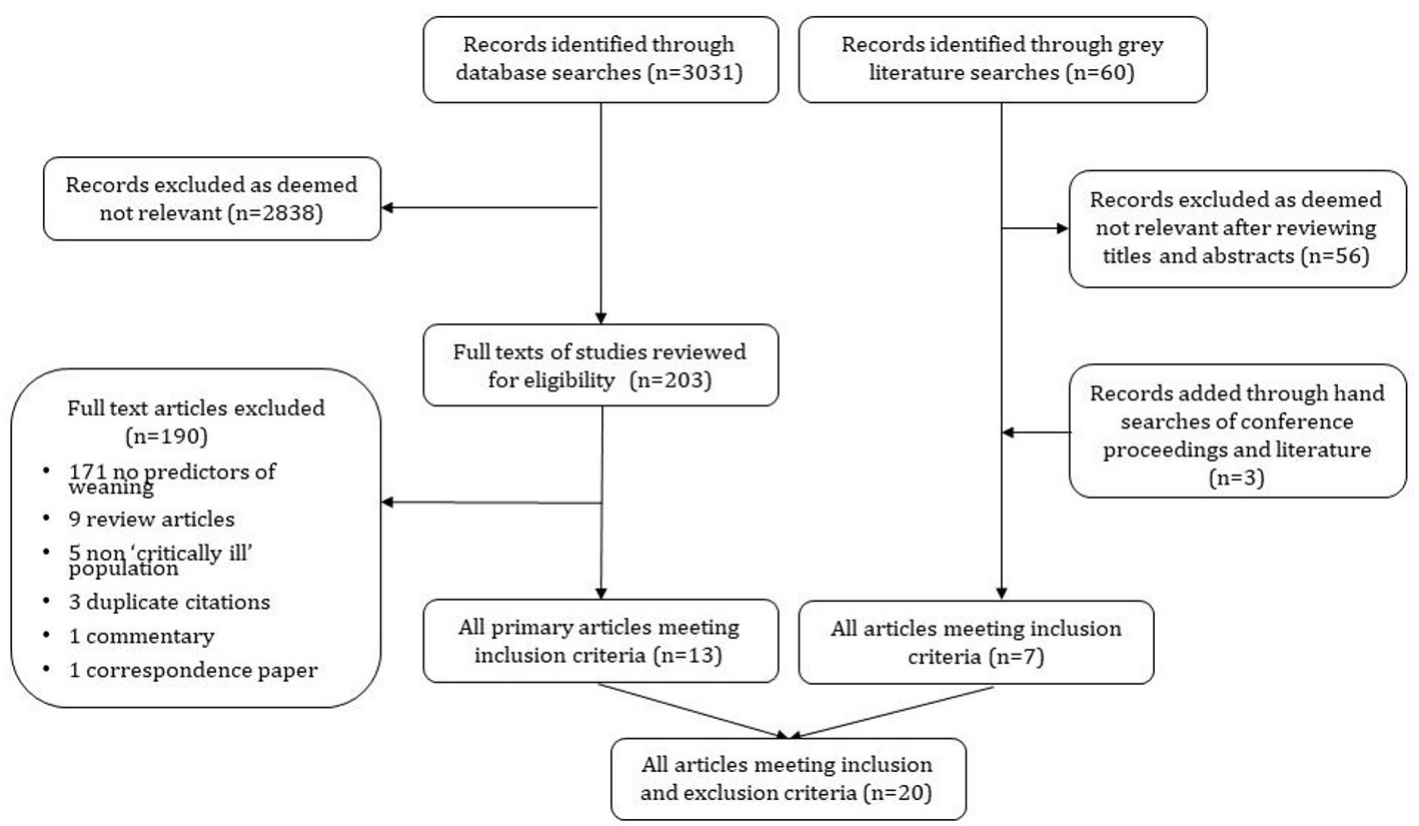

\section{References}

1. Zarbock A, Kellum J, Schidmt $C$ et al., Effect of Early vs. Delayed Initiation of Renal Replacement Therapy on Martality in Critically III Patients with Acute Kidney Injury: The ELAIN Randomized Clinical Trial, JAMA 2016;315(20): 2190-2199.

2. Gaudry S, Hajage D, Schortgen F et al., Initiation Strategies for Renal-Replacement Therapy in the Intensive Care Unit, NEJM 2016;375:122-133.3.

3. Wald R, Adhikari NK, Smith OM et al., Comparison of standard and accelerated initiation of renal replacement therapy in acute kidney injury. Kidney Int 2015;88:897-904. 


\section{Development and Evaluation of an Interactive ICU Workshop for High School} Students

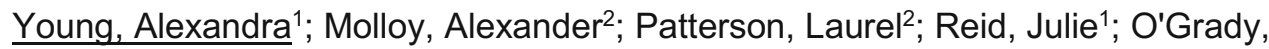
Heather $^{1}$; Seczek, Amy ${ }^{1}$; Kho, Michelle ${ }^{1,2,3}$

1 School of Rehabilitation Science, McMaster University, Hamilton, Ontario, Canada

2 Department of Physiotherapy, St. Joseph's Healthcare Hamilton, Hamilton, Ontario, Canada

3 Department of Physical Medicine and Rehabilitation, Johns Hopkins

University, Baltimore, Maryland, United States of America

Introduction: Intensive care unit (ICU) survivors are at an increased risk of disability associated with muscle wasting and ICU-acquired weakness (ICUAW) ${ }^{1-3}$. There are many multidisciplinary employment opportunities within clinical care and research that are dedicated to preventing disability and promoting recovery in ICU survivors.

Recognized team members include intensivists, nurses, and physiotherapists. Other valued team members also include respiratory and occupational therapists. In preparation for post-secondary training, Grade 11 students have a selection of healthrelated pre-requisite courses, making these students and ideal cohort to highlight the diverse career and research opportunities in the ICU. Therefore, an interactive workshop inspired by the CYCLE research program was developed to introduce Grade 11 students to clinical and research opportunities within the ICU ${ }^{4-6}$.

Objectives: To develop an interactive workshop to: 1) introduce Grade 11 high school students to career opportunities in critical care; 2 ) introduce students to common technologies and equipment in the ICU; 3 ) simulate the effects of ICUAW and introduce physiotherapy interventions to address ICUAW; 4) highlight current rehabilitation research, specifically in-bed cycling, to improve ICU survivor recovery.

Methods: We engaged a multidisciplinary team including research clinicians, research coordinators, graduate students, and a 2nd year physiotherapy student completing a clinician-scientist placement at St Joseph's Healthcare Hamilton to develop the workshop. We designed activities to encourage student participation, provide practical learning opportunities, and promote critical thinking (Table 1). An ICU summary handout was created to introduce students to the concept of critical care, related careers, and common ICU technologies. Three physiotherapists, 1 research coordinator, 1 physiotherapy student, and $1 \mathrm{MSc}$ graduate student led the workshop. Following the workshop, participants completed a self-administered, 10item questionnaire including demographics, open-ended questions, and 6 questions using a 7-point Likert scale (Table 2). We calculated the mean and standard deviation (SD) using Microsoft Excel for all interval, ordinal, and nominal data. Thematic analysis was conducted to summarize participants' responses to open-ended questions.

Results: Two teachers and 17 Grade 11 students (mean (SD) age $16.3(0.5)$ years, $79 \%$ female) from 2 Hamilton high schools participated. The overall rating of the workshop was $6.64(0.68)$. Table 1 outlines the mean (SD) ratings. Notably, participants mentioned at least 1 of the 3 interactive stations as their favourite part of the workshop (95\%). Participants recommended more interactive stations and additional emphasis on the in-bed cycling research trials. Students expressed interest in various disciplines including medicine (63\%), nursing (41\%), and allied health (29\%).

Conclusion: This 3-hour interactive workshop introduced high school students to the diverse career and research areas in critical care. The interactive stations elicited very high ratings from all students and teachers, who reported stations as understandable, organized and fun. Both students and teachers appreciated the workshop content as an opportunity to assist them in future career decisions. Results from this workshop can be applied to future knowledge translation initiatives aimed to educated high school students about the many constituents of the ICU. 
Table 1: Interactive Workshop Activity Descriptions

\begin{tabular}{|c|c|c|}
\hline Activity & $\begin{array}{l}\text { Duration } \\
\text { (minutes) }\end{array}$ & Description \\
\hline Introductory Lecture & 25 & $\begin{array}{l}\text { Students received an introduction to common } \\
\text { health care professions in the ICU, with an } \\
\text { emphasis on the role of the physiotherapist in } \\
\text { the treatment and prevention of ICU acquired } \\
\text { weakness (ICUAW). Students received an } \\
\text { overview of in-bed cycling research, including } \\
\text { the rationale to address ICUAW and improve } \\
\text { ICU patient outcomes }{ }^{1-3} \text {. }\end{array}$ \\
\hline \multicolumn{3}{|c|}{ Students rotated among the following 3 stations (groups of 5-6 students): } \\
\hline $\begin{array}{l}\text { Interactive Station 1: } \\
\text { Simulated patient } \\
\text { room }\end{array}$ & 35 & $\begin{array}{l}\text { This station consisted of two activities. Through } \\
\text { both activities, students learned how to } \\
\text { measure } \mathrm{SpO}_{2} \text { and blood pressure (BP) and } \\
\text { were introduced to common ICU technologies } \\
\text { through a scavenger hunt activity. This included } \\
\text { a simulated patient room with the technologies } \\
\text { attached to a "mannequin" patient. }\end{array}$ \\
\hline $\begin{array}{l}\text { Interactive Station 2: } \\
\text { ICU patient } \\
\text { simulation }\end{array}$ & 35 & $\begin{array}{l}\text { This station emphasized the severe physical } \\
\text { and functional implications of ICUAW through } \\
\text { student performance of common physiotherapy } \\
\text { physical mobility outcome measures ( } 30 \\
\text { second sit to stand and } 2 \text {-minute walk test) with } \\
\text { and without weighted equipment. The } \\
\text { simulation equipment was intended to restrict } \\
\text { student's physical and respiratory abilities } \\
\text { mimicking some of the limitations associated } \\
\text { with ICUAW. }\end{array}$ \\
\hline $\begin{array}{l}\text { Interactive Station 3: } \\
\text { Introduction to in-bed } \\
\text { cycling activity }\end{array}$ & 35 & $\begin{array}{l}\text { Students had the opportunity to try in-bed } \\
\text { cycling, and to discuss the evolution of in-bed } \\
\text { cycling investigations with researchers. }\end{array}$ \\
\hline Question Period & 15 & $\begin{array}{l}\text { Following the interactive stations, students } \\
\text { discussed associated health care and research } \\
\text { career opportunities with local ICU clinicians } \\
\text { and researchers from St Joseph's Healthcare } \\
\text { Hamilton. }\end{array}$ \\
\hline Feedback & 5 & $\begin{array}{l}\text { Students were given the opportunity to fill out a } \\
10 \text { item self-report questionnaire anonymously. }\end{array}$ \\
\hline Total time & 150 & \\
\hline
\end{tabular}


Table 2: Participant Ratings ( $n=19)$

\begin{tabular}{|c|l|}
\hline Item & Rating; mean (SD) \\
\hline ICU Summary Handout & $6.56(0.70)$ \\
Easy to understand & $6.83(0.38)$ \\
Learned something new & $6.44(0.92)$ \\
Well organized & $6.63(0.68)$ \\
\hline Introductory Lecture & $6.74(0.56)$ \\
Easy to understand & $6.68(0.56)$ \\
Learned something new & $6.53(0.70)$ \\
Well organized & $6.84(0.50)$ \\
\hline Interactive Station 1: Simulated patient room & $6.84(0.50)$ \\
Easy to understand & $6.79(0.54)$ \\
Learned something new & $6.68(0.82)$ \\
Well organized & $6.68(0.75)$ \\
Activities were fun & $6.79(0.54)$ \\
Easy to understand & $6.72(0.57)$ \\
Learned something new & \\
Well organized & $6.89(0.31)$ \\
Activities were fun & $6.79(0.54)$ \\
Interactive Station 2: ICU patient simulation & $6.95(0.23)$ \\
Easy to understand & $7.00(0.00)$ \\
Learned something new & $6.64(0.68)$ \\
Well organized & \\
Activities were fun & \\
\hline Overall Rating & \\
\hline
\end{tabular}

Legend: Item response for handout and interactive activities were rated on a 7 point Likert scale, where 1 point represented "strongly disagree" and 7 points represented "strongly agree". For overall ratings, 1 point represented "needs improvement" and 7 points signified "outstanding".

\section{References}

1. Herridge MS, Cheung AM, Tansey CM, Matte-Martyn A, Diaz-Granados N, Al-Saidi F, Cooper AB, Guest CB, Mazer CD, Mehta S, Stewart TE. One-year outcomes in survivors of the acute respiratory distress syndrome. New England Journal of Medicine. 2003 Feb 20;348(8):683-93.

2. Dowdy DW, Eid MP, Sedrakyan A, Mendez-Tellez PA, Pronovost PJ, Herridge MS, Needham DM. Quality of life in adult survivors of critical illness: a systematic review of the literature. Intensive care medicine. 2005 May 1;31(5):611-20.

3. Vivodtzev I, Devost AA, Saey D, Villeneuve S, Boilard G, Gagnon P, Provencher S, Simon M, Baillot R, Maltais F, Lellouche F. Severe and early quadriceps weakness in mechanically ventilated patients. Critical Care. 2014 Jun;18(3):431.

4. Kho ME, Martin RA, Toonstra AL, Zanni JM, Mantheiy EC, Nelliot A, Needham DM. Feasibility and safety of in-bed cycling for physical rehabilitation in the intensive care unit. Journal of critical care. 2015 Dec 1;30(6):1419-el.

5. Kho ME, Molloy AJ, Clarke FJ, Ajami D, McCaughan M, Obrovac K, Murphy C, Camposilvan L, Herridge MS, Koo KK, Rudkowski J. TryCYCLE: a prospective 
study of the safety and feasibility of early in-bed cycling in mechanically ventilated patients. PloS one. 2016 Dec 28;11(12):e0167561.

6. Kho ME, Molloy AJ, Clarke F, Herridge MS, Koo KK, Rudkowski J, Seely AJ,

Pellizzari JR, Tarride JE, Mourtzakis M, Karachi T. CYCLE pilot: a protocol for a pilot randomised study of early cycle ergometry versus routine physiotherapy in mechanically ventilated patients. BMJ open. 2016 Apr 1;6(4):e011659. 
Diarrhea During Critical IIIness: An Interim Analysis

Dionne, Joanna C. ${ }^{1,2,4}$; Sullivan, Kristen ${ }^{3}$; Mbuagbaw, Lawrence ${ }^{1}$; Takaoka, Alyson $^{1}$; Duan, Erik H. ${ }^{2}$; Alhazzani, Waleed2; Devlin, John W. ${ }^{7}$; Duprey, Matthew ${ }^{7}$; Moayyedi, Paul4; Armstrong, David"; Thabane, Lehana ${ }^{4}$; Tsang, Jennifer2; Jaeschke, Roman²; Karachi, Timothy'; Hamielec, C. ${ }^{2}$; Cartin-Ceba, Rodrigo ${ }^{5}$; Muscedere, John ${ }^{6}$; Alshahrani, Mohammed ${ }^{8}$; Cook, Deborah ${ }^{1,2}$.

1 Department of Health Research Methods, Evidence and Impact, McMaster

University, Hamilton, Ontario, Canada

2 Department of Medicine, Division of Critical Care Medicine, McMaster University, Hamilton, Ontario, Canada

3 Department of Medicine, McMaster University, Hamilton, Ontario, Canada

4 Department of Medicine, Division of Gastroenterology, McMaster University, Hamilton, Ontario, Canada

5 Mayo Clinic, Phoenix, Arizona, USA;

6 Department of Critical Care Medicine, Queen's University, Kingston, Ontario,

Canada

7 Department of Pharmacy and Health Systems Sciences, Northeastern University, Bostom, Massachuettes, USA

8 College of Medicine, University of Dammam, Dammam, Saudi Arabia

Introduction: Diarrhea is a frequent concern in the Intensive Care Unit (ICU) and its incidence varies from $2-95 \%$. This variation may be due to the absence of a consistent definition of diarrhea and inattention to this clinical problem.

Objectives: The objectives of this study were to describe the epidemiology of diarrhea in critically ill patients including the incidence, risk factors, and consequences of diarrhea, and the incidence of Clostridium difficile-associated diarrhea.

Methods: This prospective cohort study was undertaken over 10 weeks in 9 ICUs in Canada and the United States. We included all patients $>18$ years old who were admitted to the ICU for $>24$ hours and followed them daily until ICU discharge. The bedside nurse documented all bowel movements, classifying them using the Bristol and Bliss Stool Charts. We defined diarrhea in 3 ways: 1) WHO Criteria of $>3$ liquid bowel movements per day (Bristol type 7), 2) any Bristol type 6 or 7 stool, and 3) any Bliss score of 4 . Daily data on pertinent life support, laboratory values (e.g., electrolytes), treatment (e.g., medications, fecal management devices, nutrition), and outcomes (including mortality) were evaluated using multivariate methods.

Results: Among 865 patients, the incidence of diarrhea varied based on the definition (WHO Criteria: $77.3 \%$; 95\% Cl 774.4-80.0, Bristol Stool Chart definition: 56.8\%; 95\% Cl 53.5-60.1, Bliss Stool Chart definition: 39.9\%; 95\% Cl 36.7-43.2). Risk factors associated with diarrhea on multivariable analysis included total number of antibiotic days OR $1.17(95 \% \mathrm{Cl} 1.10-1.24)$ use of sorbitol-containing medications $1.76(95 \% \mathrm{Cl}$ 1.27-2.45) and enteral nutrition OR 2.24 (95\% Cl 1.56-3.22), while opiates were associated with a lower risk OR $0.22(95 \% \mathrm{Cl} 0.13-0.36)$. Diarrhea often prompted cessation of enteral nutrition, prokinetics or stool softeners, or fecal management device insertion. Overall mortality was not different between diarrhea $(78.6 \%)$ and nondiarrhea $(73.8 \%)$ groups $(p=0.184)$. Only $18 / 56(32 \%)$ of Clostridium difficile toxin positive patients met the definition of Clostridium difficile-associated diarrhea.

Conclusion: In this multicenter cohort of critically ill patients, diarrhea occurred frequently; although the reported incidence differed based on the definition employed. Clostridium difficile-associated diarrhea was rare. Antibiotics exposure, hyperosmolar medications use and enteral nutrition increased the risk of diarrhea; opiates reduced it. 


\section{Diarrhea During Mechanical Ventilation: A Multicenter Study}

Dionne, Joanna C. ${ }^{1}$; Duan, Erick H. ${ }^{1}$; Clarke, France ${ }^{2}$; Hand, Lori' ${ }^{2}$ Millen, Tina ${ }^{2}$; Sandu, Gyan'; Hodder, Jennifer ${ }^{2}$; Santos, Marlene ${ }^{2}$; Shah, Sumesh'; Trembley, MarieClaude $^{2}$; Gomes, Bridgett²; Leclair, Liane'; Montroy, Kaitlyn²; Watpool, Irene ${ }^{2}$; Porteous, Rebecca²; Arces, Shelley²; Foster, Denise ${ }^{2}$; Auld, Fiona ${ }^{2}$; Williams, Virginie2; Marchand, Joannie ${ }^{2}$; Campisi, Josie ${ }^{2}$; Alam, Norine ${ }^{2}$; Lebrassier, Martine ${ }^{2}$; Thompson, Patricia2; Hewer, Tayne²; Gilles, Diana²; Hunt, Miranda²; Georgescu, llinca²; Boyd, Tracy $^{2}$; Lys, Justin²; Marten, Nicole ${ }^{2}$; Campbell, Eileen²; Bentall, Tracey ${ }^{2}$; Kavikondala, Kanthi²; Willems, Suzette'; Panchbhaya, Zaynab²; Booth, Josh²; Ruddell, Stacey²; Richter, Beverly2; Tassy, Danae ${ }^{2}$; Jesso, Rebecca 2; Marinoff, Nicole²; Perez, Adic; Kaur, Navjot ${ }^{2}$; Campbell, Tracy²; Lizotte, Patricia²; Lavoie, Lise²; Dionne, Maude 2; Saunders, Lois ${ }^{1}$; Zytaruk, Nicole ${ }^{1}$; Heels-Ansdell, Diane ${ }^{3}$; Johnstone, Jennie ${ }^{4}$; Cook, Deborah $^{1,3}$

1 Department of Medicine, Division of Critical Care, McMaster University, Hamilton, Ontario, Canada

2 Canadian Critical Care Trials Group Research Coordinators Group, Montreal, Quebec, Canada

3 Department of Health Research Methods, Evidence and Impact, McMaster University, Hamilton, Ontario, Canada

4 Dalla Lama School of Public Health, University of Toronto, Toronto, Ontario, Canada

Background: Diarrhea is of interprofessional concern in daily ICU care, often associated with changes in drugs, diet, and can result in the insertion of rectal devices. The objective of this nested sub study within the PROSPECT Trial was to describe the incidence, risk factors for diarrhea in mechanically ventilated patients at the two-thirds enrolment mark.

Methods: PROSPECT Research Coordinators collected baseline and daily data on patients enrolled in a randomized placebo-controlled trial testing the effect of twice daily Lactobacillus rhamnosus GG on infectious outcomes and diarrhea. Patients were considered as a cohort rather than in 2 groups. Bedside Nurses prospectively documented daily bowel movements [BMs], recording the number, frequency, and Bristol Stool Form type. We defined diarrhea as either $>3 B M s$ per day or $>1$ Bristol type 6 or 7 stool.

Results: Among 1,243 invasively mechanically ventilated patients, $70.2 \%$ received inotropes, $13.4 \%$ received renal replacement therapy and $1.5 \%$ were on extracorporeal membrane oxygenation (ECMO) at some time in ICU. The mean age was 60.3 (standard deviation [SD] 16.4) years, and the mean APACHE II Score was 22.1 (SD 8.0). Over 16,685 ICU patient-days, every BM was characterized. The incidence of diarrhea was $59.5 \%$ when defined as $>3$ Bristol Type 6/7 BMs on any day, and $84.2 \%$ when defined as $>1$ Bristol Type 6-7 stool on any day. No BMs were passed on 5,985 patient-days. Multivariate Cox proportional hazards regression showed that age, illness severity and history of Clostridium difficile were not associated with diarrhea. However, frailty (Hazard Ratio [HR] 1.21, 95\% Cl 1.04-1.42) and prior gastrointestinal disease (inflammatory bowel disease, irritable bowel syndrome or celiac disease) (HR $1.77,95 \% \mathrm{Cl} 1.21-2.60)$ significantly increased the risk. In terms of daily events and exposures, advanced life supports (vasopressors and renal replacement therapy) and motility agents (prokinetics and opiates) were not associated with diarrhea. However, antibiotics (HR 1.70, 95\% Cl 1.38-2.09), enteral nutrition ( $\mathrm{HR} 1.35,95 \% \mathrm{Cl} 1.01-1.79)$, laxatives (HR 2.11, 95\% Cl 1.75-2.53), and ICU-acquired Clostridium difficile infection (HR 3.48, 95\% Cl 1.75-6.93) significantly increased the risk of diarrhea. Acid suppression with proton pump inhibitors were associated with diarrhea (HR 1.29, $95 \% \mathrm{Cl} 1.06-1.57)$, whereas histamine-2-receptor antagonists were not (HR 1.11, 95\% Cl 0.91-1.36).

Conclusions: This analysis shows that diarrhea affects the majority of mechanically 
ventilated patients. While some baseline factors increase the chance of diarrhea during critical illness (e.g., frailty, and gastrointestinal comorbidity associated with diarrhea), some potentially modifiable time-dependent factors also increase the risk (e.g., antibiotics, enteral nutrition, Clostridium difficile infection and proton pump inhibitors). 
Do Social Determinants Increase the Risk of an In-Hospital Critical Event When Admitted to Medicine or Surgery?

Lee, David ${ }^{1}$; Skitch, Steven²; Xu, Michael K3 ${ }^{3}$; Fox-Robichaud, Alison E ${ }^{4}$

1 Bachelor of Health Sciences (Honours) Program, McMaster University, Hamilton, Ontario, Canada

2 Department of Medicine, Divisions of Critical Care and Emergency Medicine, McMaster University Hamilton, Ontario, Canada

3 Health Economics Associate, Pivinia Consulting Inc., Mississauga, Ontario, Canada 4 Department of Medicine, Division of Critical Care, McMaster University and Hamilton Health Sciences, Hamilton, Ontario, Canada

Introduction: The Hamilton Early Warning Score (HEWS) is an electronic bedside tool that uses vital sign abnormalities to detect potential critical events. HEWS at ED triage had poor (AUROC 0.62) discriminative ability for predicting the likelihood of critical event during subsequent hospital stay but better discrimination for critical events in patients who are septic (AUROC 0.82). ${ }^{1,2}$ New evidence suggests using social determinants may assist in predicting adverse events. A Danish study found that among septic ICU patients, low income was significantly associated with increased 30day mortality. ${ }^{3}$ In 2010, McMaster University and the Hamilton Spectator collaborated on the Code Red project which described the disparities in the determinants of health and health status that exists in the City of Hamilton's neighbourhoods. ${ }^{4,5}$

Objectives: The primary objective of our study was to determine whether the addition of social determinants such as postal code and income level add predictive value to the current HEWS.

Methods: The study population was derived from an existing database of patients admitted to one of 8 adult medical or surgical wards at the Hamilton General and Juravinski hospitals over a 6-month period from January to June 2015. Cases experienced a critical event defined as an unplanned intensive care unit admission, cardiopulmonary resuscitation, or unexpected death. Controls were matched to cases in a 2-to-1 ratio controlling for comorbidities defined by their Charlson Comorbidity Index. To this case-control data was added the first half of the postal code and the income tertiles for these areas of Hamilton. We used the 2010 Hamilton Code Red report to define postal codes residing in higher risk areas. ${ }^{4,5}$ Conditional Regression was used to evaluate the correlation between HEWS at ED triage, residing in a highrisk area, and income tertiles as a predictor for critical events.

Results: The cohort consisted of 798 patients 270 of whom experienced a critical event $(45.1 \%)$. Chi-Square analysis identified that residing in Code Red areas $(p=0.01)$ and an elevated HEWS at ED triage $(p=0.00)$ as significant. Conditional regression analysis identified Code Red areas $(p=0.02, R R=1.81)$ and HEWS $(p=0.03, R R=1.27)$ but not income tertiles as predictors for critical events.

Conclusion: We found that residing in a Code Red area within Hamilton placed patients admitted to a medicine or surgical ward at increased risk for a critical event. Income level, as determined by one's postal code was not found to be a predictor for critical event. Further investigation into the utility of early warning scores should consider other social determinants such as education and housing quality as well access to a primary care physician and transportation.

\section{References}

1. Tam B, Xu M, Kwong M, Wardell C, Kwong A, Fox-Robichaud A. The Admission Hamilton Early Warning Score (HEWS) Predicts the Risk of Critical Event during Hospitalization. Canadian Journal of General Internal Medicine. 2017 Feb 24;11(4). 
2. Skitch S, Tam B, Xu M, McInnis L, Vu A, Fox-Robichaud A. Examining the utility of the Hamilton early warning scores (HEWS) at triage: Retrospective pilot study in a Canadian emergency department. Canadian Journal of Emergency Medicine. 2017 May:1-9

3. Schnegelsberg A, Mackenhauer J, Nibro HL, Dreyer P, Koch K, Kirkegaard H. Impact of socioeconomic status on mortality and unplanned readmission in septic intensive care unit patients. Acta Anaesthesiologica Scandinavica. $2016 \mathrm{Apr}$ 1;60(4):465-75.

4. DeLuca, PF, Buist, S, Johnston, N (2012). Engaging Communities in Health System Change - The Code Red Project in Hamilton, Canada. Social Indic Res, 108(2):317327.

5. Buist S, and Johnston N. https://www.thespec.com/hamilton-topics/3236468-codered-hamilton/ Accessed April 2, 2018 


\section{Effect of Graded Passive Cycling on Cerebral Blood Flow, Cardiac Function, and Global Hemodynamics in Sepsis}

Chen, Jennifer ${ }^{1}$; Martin, Claudio2; Mclntyre ${ }^{1,2}$, Chris; Ball, lann ${ }^{2,3}$; Slessarev, Marat ${ }^{1,2}$ 1 Departments of Medical Biophysics, Western University, London, Ontario, Canada 2 Department of Medicine, Western University, London, Ontario, Canada

3 Department of Epidemiology and Biostatistics, Western University, London, Ontario, Canada

Introduction: Passive cycling (PC) is a practical intervention that may improve functional outcomes and decrease pain in septic patients, possibly by reducing inflammation (Amidei, 2013) in early stages of sepsis when active exercise is not feasible due to sedation. It is a safe and feasible intervention in intensive care units (ICU) (Pires-Neto, 2013) despite increasing oxygen consumption in mechanically ventilated patients (Savi, 2010). However, while it can benefit patients by preserving muscle mass and strength (Hickmann, 2018), it can potentially lead to impaired distal organ perfusion. Furthermore, the effective dose of PC may vary between patients. The effect of PC on distal organ perfusion, and variation in patient responses to differing intensities of passive exercise remains unknown. These factors are critical to establishing the safety and appropriate dosage of PC in individual critically ill patients during the acute phase of their illness.

Objectives: 1) To observe the effect of PC on global hemodynamics, cerebral blood flow, and heart function in septic patients. 2) To observe the optimal intensity of PC in patients.

Methods: We used Finapres $®$ NOVA and intravenous monitoring, transcranial Doppler, and speckle-tracking echocardiography to measure global hemodynamics, middle cerebral artery velocity (MCAv, an indicator of cerebral blood flow) and cardiac function (left ventricular ejection fraction [EF] and global longitudinal strain [GLS]) during incremental increases of in-bed PC intensity in septic patients. The protocol started and ended with 5 minutes of baseline measurements at rest. PC intensity was progressively increased from 5 to 55 rotations per minute (RPM) in 10 RPM increments, with each experimental stage lasting 5 minutes. Mean values were calculated for all measured parameters during the last 2 minutes of each experimental stage. ANOVA was used to determine difference between experimental stages both within and between patients.

Results: Ten septic patients (6 males, 4 females: age $53.3 \pm 9.8$ years) completed the protocol within 1-3 days of sepsis onset. Increasing PC intensity had no effect on global hemodynamics, but was associated with a dose-dependent decrease in MCAv, and an improvement in both EF and GLS. The changes in MCAv, EF, and GLS were not uniform across patients, occurring at different PC intensities in some, while not changing in others.

Conclusions: In contrast to healthy volunteers (Chen et al, 2017), increasing PC intensity in septic patients is associated with a dose-dependent decrease in cerebral blood flow and improvement in heart function, despite stable hemodynamics.

Furthermore, the PC intensity that results in these changes varies between patients. Our results are the first to show that in-bed PC can induce changes in distal organ function, improving heart contractility in a dose-dependent manner. However, this may come at the expense of brain perfusion. The PC intensity dose that results in these changes differs between patients, suggesting the need for its individualized prescription in individual patients. 


\section{References}

1. Amidei, C. \& Sole, M. L. Physiological Responses to Passive Exercise in Adults Receiving Mechanical Ventilation. Am J Crit Care 22, 337-348 (2013).

2. Hickmann, C. E. et al. Impact of Very Early Physical Therapy During Septic Shock on Skeletal Muscle: A Randomized Controlled Trial. Crit. Care Med. (2018). doi:10.1097/CCM.0000000000003263

3. Pires-Neto, R. C. et al. Very Early Passive Cycling Exercise in Mechanically Ventilated Critically III Patients: Physiological and Safety Aspects - A Case Series. PLOS ONE 8, e74182 (2013).

4. Savi, A., Maia, C. P., Dias, A. S. \& Teixeira, C. Hemodynamic and metabolic effects of passive leg movement in mechanically ventilated patients. Rev Bras Ter Intensiva 22, 315-320 (2010).

5. CCCF 2017 Abstracts. Can J Anesth/J Can Anesth 1-131 (2018). doi:10.1007/s12630-018-1162-7 


\section{Embrionic-Derived $\mathrm{Myb}^{-}$Macrophages Improve Recovery and Reduce Bacterial Burden in Rodent Polymicrobial Abdominal Sepsis}

Jerkic, Miriana $^{1}$; Gagnon, Stéphane ${ }^{1}$; Otulakowski, Gail2; Litvack, Michael³; Post, Martin ${ }^{3}$; Kavanagh, Brian ${ }^{2,4}$; Laffey G, John ${ }^{1,5}$

1 Department of Anaesthesia, Keenan Research Centre of St. Michael's Hospital, Toronto, Canada

2 Translation Medicine, Hospital for Sick Children, Toronto, Ontario, Canada

3 Program of Physiology and Experimental Medicine, Hospital for Sick Children, Toronto, Ontario, Canada

4 Departments of Critical Care and Anesthesia, Hospital for Sick Children, University of Toronto, Ontario, Canada

5 Department of Anaesthesia, School of Medicine and Regenerative Medicine Institute, National University of Ireland, Galway, Ireland

Introduction \& Objective: Sepsis and septic shock are characterized by a dysregulated immune response to microbial invasion ${ }^{1}$. Bacterial infection of the lung and abdominal cavity constitute the commonest causes of sepsis ${ }^{2}$. In the peritoneal cavity two types of macrophages $(\mathrm{M} \phi)$ are recognized: large Embryonic derived (Ed) Myb independent M $\phi$ or LPMs, crucial for tissue homeostasis, mainly replaced by proinflammatory small M $\phi$ or SPMs originated from circulating monocytes under inflammatory conditions. Myb ${ }^{-} \mathrm{M} \phi$ derived from pluripotent stem cells (PSCs) have demonstrated therapeutic benefit in experimental pulmonary transplantation and animal models of acute and chronic airway diseases ${ }^{3}$. We wished to determine the potential of intra-peritoneal Ed-M $\phi$ delivery in a rodent polymicrobial abdominal sepsis.

Methods: Directed differentiation of murine PSCs was used to produce expandable $E d-M \phi$ conditioned to a 'LPM-peritoneal-like' phenotype with granulocyte-macrophage colony-stimulating factor. Polymicrobial sepsis was developed in rats by instilling cecal slurry intraperitoneally (i.p., $0.5 \mathrm{~g} / \mathrm{kg})$. Ed-M $\phi\left(10 \times 10^{\wedge} 6 \mathrm{cells} / \mathrm{kg}\right)$ or vehicle (PBS) were given i.p. to the rats $4 \mathrm{~h}$ later and the animals were monitored for 48 or 72 hours. Lung functional parameters, broncho-alveolar lavage (BAL), peritoneal lavage fluid (PLF) and major organs were collected for infiltration and bacterial count assessment. Macrophages (Mf) and neutrophils (Nf) were isolated from the peritoneum of septic animals using Ficoll gradient and their phagocytic function (using serum opsonized zymosan), activation state, reactive oxygen species (ROS) production, as well as Mf efferocytosis determined by confocal microscopy. Nf apoptotic markers were assessed by Western blot analysis.

Results: Ed-M $\phi$ treatment significantly attenuated the increase in alveolar and peritoneal white blood cell and neutrophil infiltration induced following abdominal fecal sepsis. Ed-M $\phi$-treated rats cleared bacteria more efficiently, with lower bacterial counts (CFU/mL in the lung, liver \& spleen compared to vehicle-treated animals). Peritoneal $\mathrm{M} \phi$ isolated from septic rats treated with $\mathrm{Ed}-\mathrm{M} \phi$ showed better attachment, more effective phagocytosis and higher ROS production, compared to M $\phi$ 's from vehicle treated animals. In contrast, peritoneal $\mathrm{Nf}$ isolated from septic rats treated with vehicle were more active and produced more ROS than Nf isolated from septic rats treated with Ed-M $\phi$. Apoptotic markers (Caspase-3 and Bax) were significantly more expressed in PLF Nf from Ed-M $\phi$ treated than vehicle treated septic rats $72 \mathrm{~h}$ after sepsis induction. That indicates prolonged life span of PLF Nf from PBS-treated rats, as another sign of more severe sepsis ${ }^{4}$ in this animal group.

Conclusions: Intra-peritoneal Ed-Mf therapy attenuated the severity of fecal sepsis, and reduced bacterial load, and may have therapeutic potential for systemic sepsis. 


\section{References}

1. Seymour CW, Liu VX, Iwashyna TJ, et al.: Assessment of Clinical Criteria for Sepsis: For the Third International Consensus Definitions for Sepsis and Septic Shock (Sepsis-3). JAMA, 315(8):762, 2016.

2. Keane C, Jerkic M, and Laffey JG: Stem Cell-based Therapies for Sepsis. Anesthesiology, 127(6), 1017-34, 2017.

3. Litvack ML, Wigle TJ, Lee J, et al.: Alveolar-like Stem Cell-derived Myb(-) Macrophages Promote Recovery and Survival in Airway Disease. Am J Resp Crit Care Med, 193(11):1219-29, 2016.

4. Shen XF, Cao K, Jiang JP et al.: Neutrophil dysregulation during sepsis: An overview and update. J Cell Mol Med, 21(9), 1687-97, 2017. 
End of Life Skills \& Professionalism for Critical Care Residents in Training (ESPRIT): A Canada-Wide Educational Survey

Arora, $\mathrm{SA}^{1,2,3}$; Shaikh, $\mathrm{S}^{4}$; Karachi, $\mathrm{T}^{4}$; Centofanti, J4,5; Meade, $\mathrm{MO}^{3,4}$; Piquette, $\mathrm{D}^{7}$; Woods, $A^{6}$; Boyle, $A^{6}$; Downar, J J,8; Cook, $\mathrm{DJ}^{3,4}$

1 Division of Critical Care, Department of Medicine, Northern Ontario School of

Medicine, Thunder Bay, Ontario, Canada

2 Division of Critical Care, Department of Medicine, Mackenzie Health, Toronto,

Ontario, Canada

3 Department of Health Research Methods, Evidence, and Impact, McMaster

University, Hamilton, Ontario, Canada

4 Department of Critical Care, McMaster University, Hamilton, Ontario, Canada

5 Department of Anesthesia, McMaster University, Hamilton, Ontario, Canada

6 Department of Palliative Care, McMaster University, Hamilton, Ontario, Canada

7 Department of Critical Care, University of Toronto, Toronto, Ontario, Canada

8 Department of Palliative Care, University of Toronto, Toronto, Ontario, Canada

Introduction/Background: End of life (EOL) care is a pivotal component of comprehensive care for critically ill patients. EOL care in the ICU includes providing grieving patients and families with medical, psychological, and spiritual support, as well as supporting the patient or family's wishes for organ donation when death is a probable or expected outcome. To prepare future critical care medicine (CCM) physicians to provide this care, training programs should include teaching on EOL care as part of their formal curricula.

Objectives: The objective of the ESPRIT Study (End of Life Skills \& Professionalism for Critical Care Residents in Training) was to conduct a cross-sectional survey of Canadian CCM residents and program directors to describe and analyze current educational practices about EOL care.

Methods: We identified survey topics and items following a focused literature review related to each critical care national objective of training. We then created questions about each topic to address 3 domains of interest: 1) curricular content \& evaluation methods, 2) resident's preparedness to meet these objectives, and 3) how learning could be improved. We formatted a self-administered instrument tailored to each respondent group, performed pilot testing for each instrument, and used a Clinical Sensibility Questionnaire to test our resident survey instrument. We then distributed the ESPRIT Survey to all CCM residents and program directors across the $13 \mathrm{CCM}$ programs in Canada.

Results: Our national response rate was $82 \%(n=78)$ for the resident survey and $92 \%$ $(n=12)$ for the program director survey. For 7 of $8 \mathrm{EOL}$ learning objectives, residents reported direct observation as the teaching modality used most effectively in their training, followed by informal advice and academic half-day teaching. For non-donation EOL objectives, residents reported most comfort on a 5-point Likert scale with withdrawal of life-sustaining therapy (WLST) (mean 3.6; SD 0.8) and pain \& symptom management (mean 3.6; SD 0.8), and the least comfort with cultural awareness (mean 3.2; SD 1). For donation-related skills, residents were most comfortable identifying neurologically deceased potential donors (NDDs) (mean 3.6; SD 1), and least comfortable with conducting a donation after cardiac death (DCD) (mean 2.5, SD 1.1). Similarly, program directors cited academic-half day, direct observation, and palliative care experiences as the most commonly used teaching modalities to address EOL learning objectives in their program. Program directors ranked WLST as the most effectively taught objective (mean 3.67; SD 0.7), and cultural awareness as least effectively taught (mean 2.67; SD 0.8). For donation-related objectives, program directors ranked declaration of death by neurologic criteria as the most effectively taught (mean 4.5; SD 0.7), and discussing DCD with families as least effectively taught (mean 2.67; SD 1.2). 
Conclusions: EOL learning objectives in Canada are currently most effectively taught through direct observation, informal advice, and academic-half day sessions. Residents self-report a modest level of comfort with all EOL teaching objectives except for those related to donation after cardiac death- an area which program directors also identify as requiring more effective teaching. Critical care medicine residency programs may consider implementing a comprehensive EOL curriculum including focus on these objectives. Particular attention should be devoted to organ donation to ensure these competencies are attained.

\section{References}

1. Aslakson RA, Curtis JR, Nelson JE. The changing role of palliative care in the ICU. Crit Care Med 2014;42 11: 2418-28.

2. Bekelman JE, Halpern SD, Blankart CR, Bynum JP, Cohen J, Fowler R et al. Comparison of Site of Death, Health Care Utilization, and Hospital Expenditures for Patients Dying With Cancer in 7 Developed Countries. JAMA 2016;315 3: 272-83.

3. Heyland DK, Lavery JV, Tranmer JE, Shortt SE, Taylor SJ. Dying in Canada: is it an institutionalized, technologically supported experience? J Palliat Care 2000;16 Suppl: S10-6.

4. Somogyi-Zalud E, Zhong Z, Hamel MB, Lynn J. The use of life-sustaining treatments in hospitalized persons aged 80 and older. J Am Geriatr Soc 2002;50 5: 930-4.

5. Heyland DK, Dodek P, Rocker G, Groll D, Gafni A, Pichora D et al. What matters most in end-of-life care: perceptions of seriously ill patients and their family members. CMAJ 2006;174 5: 627-33.

6. Malacrida R, Bettelini CM, Degrate A, Martinez M, Badia F, Piazza J et al. Reasons for dissatisfaction: a survey of relatives of intensive care patients who died. Crit Care Med 1998;26 7: 1187-93.

7. Azoulay E, Chevret S, Leleu G, Pochard F, Barboteu M, Adrie C et al. Half the families of intensive care unit patients experience inadequate communication with physicians. Crit Care Med 2000;28 8: 3044-9.

8. Saft HL, Richman PS, Berman AR, Mularski RA, Kvale PA, Ray DE et al. Impact of critical care medicine training programs' palliative care education and bedside tools on ICU use at the end of life. J Grad Med Educ 2014;6 1: 44-9.

9. Hill AD, Fan E, Stewart TE, Sibbald WJ, Nauenberg E, Lawless B et al. Critical care services in Ontario: a survey-based assessment of current and future resource needs. Can J Anaesth 2009;56 4: 291-7.

10. Hinkle LJ, Bosslet GT, Torke AM. Factors associated with family satisfaction with end-of-life care in the ICU: a systematic review. Chest 2015;147 1: 82-93.

11. Downar J, Knickle K, Granton JT, Hawryluck L. Using standardized family members to teach communication skills and ethical principles to critical care trainees. Crit Care Med 2012;40 6: 1814-9.

12. Arnold RM, Back AL, Barnato AE, Prendergast TJ, Emlet LL, Karpov I et al. The Critical Care Communication project: improving fellows' communication skills. J Crit Care 2015;30 2: 250-4.

13. Hope AA, Hsieh SJ, Howes JM, Keene AB, Fausto JA, Pinto PA et al. Let's Talk Critical. Development and Evaluation of a Communication Skills Training Program for Critical Care Fellows. Ann Am Thorac Soc 2015;12 4: 505-11.

14. McCallister JW, Gustin JL, Wells-Di Gregorio S, Way DP, Mastronarde JG. Communication skills training curriculum for pulmonary and critical care fellows. Ann Am Thorac Soc 2015;12 4: 520-5.

15. Sprung CL, Cohen R, Marini JJ. The top attributes of excellence of intensive care physicians. Intensive Care Med 2015;41 2: 312-4.

16. Burns KE, Duffett M, Kho ME, Meade MO, Adhikari NK, Sinuff T et al. A guide for the design and conduct of self-administered surveys of clinicians. CMAJ 2008;179 3: 245-52. 
Epidemiologic Risk Factors of Antimicrobial Resistance in Patients with Septic Shock Admitted to North American Critical Care Units: A Retrospective Cohort

Hewer, Tayne ${ }^{1}$; Karvellas, Constantine $\mathrm{J}^{2}$; Kutsogiannis, Demetrios $\mathrm{J}^{2}$; Kumar, Anand ${ }^{3}$

- in collaboration with the Cooperative Anti-microbial Therapy of Septic Shock (CATSS)

Database working group

1 Department of Medicine, Faculty of Medicine and Dentistry, University of Alberta, Edmonton, Alberta, Canada

2 Department of Critical Care Medicine, Faculty of Medicine and Dentistry, University of Alberta, Edmonton, Alberta, Canada

3 Sections of Critical Care Medicine and Infectious Diseases, University of Manitoba, Winnipeg, Manitoba, Canada

Introduction: Antibiotic resistance is a serious global threat resulting in a significant clinical and economic burden. Identifying resistance between different epidemiological groups is required to identify sub-populations of ICU patients who may be more susceptible to life-threatening infections caused by resistant pathogens.

Objectives: The specific aim of this retrospective cohort study was to determine the frequency of resistant organisms among different epidemiological sub-groups of patients within the Cooperative Anti-microbial Therapy of Septic Shock (CATSS) Database with an ICU admission diagnosis of septic shock.

Methods: We conducted a retrospective review of a critically ill patients with septic shock within the CATSS Database between 1996 and 2012. The presence or absence of resistant organisms was pre-specified. Multivariable logistic regression analysis was used to describe independent predictors of the presence of antibiotic resistance and the effect of antibiotic resistance on ICU and hospital mortality among patients with septic shock.

Results: Increasing age (OR $1.0095 \% \mathrm{Cl} 1.00,1.03)$, liver failure (OR 1.23 95\% Cl $1.03,1.46)$, ventilator dependence (OR $2.0995 \% \mathrm{Cl} 1.16,3.76$ ) diabetes mellitus requiring insulin (OR $1.2295 \% \mathrm{Cl} 1.04,1.42)$, elective surgery (OR $1.3395 \% \mathrm{Cl}$ $1.17,1.52)$ and emergent surgery (OR $1.2495 \% \mathrm{Cl} 1.05,1.48)$, neuromuscular disease (OR $1.5495 \% \mathrm{Cl} 1.15,2.06$ ) and nosocomial acquired infection (OR $1.7095 \% \mathrm{Cl}$ $1.52,1.90$ ) were independent predictors of the increased odds of the presence of any resistant organism at the time of diagnosis of septic shock. Leukemia (OR $0.8095 \% \mathrm{Cl}$ $0.64,1.00$ ) and the history of hypertension (OR $0.86,95 \% \mathrm{Cl} 0.78,0.96$ ) were independent predictors of reduced odds of the presence of any resistant organism at the time of diagnosis of septic shock. In the multivariable model, the presence of any antibiotic resistant organism was not an independent predictor of either ICU or hospital mortality. However, increasing age (OR 1.03 95\% Cl 1.02,1.03), APACHE (OR 1.10 $95 \% \mathrm{Cl} 1.09,1.11$ ), AIDS (OR 2.32 95\% Cl 1.65,3.26), lymphoma (OR $1.9995 \% \mathrm{Cl}$ $1.47,2.69)$ metastatic cancer (OR $1.3695 \% \mathrm{Cl} 1.15,1.62)$, liver failure (OR 3.36, 95\% $\mathrm{Cl} 2.70,4.18$ ) chronic dialysis (OR $1.4095 \% \mathrm{Cl} 1.09,1.79$ ) emergent surgery (OR 1.34 $95 \% \mathrm{Cl} 1.090,1.65)$ ICU admission source from a medical ward (OR $1.4495 \% \mathrm{Cl}$ $1.24,1.67$ ) and nosocomial acquired infection (OR $2.0495 \% \mathrm{Cl} 1.78,2.33$ ) were independent predictors of increased odds of hospital mortality. Admission source from the emergency room (other hospital ER OR $0.54,95 \% \mathrm{Cl} 0.44,0.67$ or the same hospital ER OR $0.7395 \% \mathrm{Cl} 0.62,0.85$ ), and history of hypertension (OR $0.82,95 \% \mathrm{Cl}$ $0.72,0.92$ ) were independent predictors of reduced odds of hospital mortality.

Conclusion: Increasing age, liver failure, ventilator dependence, insulin dependent diabetes, elective and emergent surgery, neuromuscular disease and nosocomial acquired infection were predictors of increased odds of the presence of antibiotic resistance at the time of septic shock. Increasing age, APACHE, AIDS, lymphoma, metastatic cancer, liver failure, chronic dialysis, emergent surgery medical ward admission source and nosocomial acquired infection were independent predictors of hospital mortality. Hypertension and ER origin were independent predictor of reduced 
odds of hospital mortality. We did not show a significant relationship between the presence of any antibiotic resistant organism and mortality.

Table 1 - Multivariable Logistic Regression for Independent Predictors of the Prevalence of Antibiotic Resistance at the Time of Septic Shock Diagnosis

\begin{tabular}{|c|c|c|c|c|}
\hline $\begin{array}{l}\text { Patient } \\
\text { Characteristic }\end{array}$ & $\begin{array}{l}\text { Univariable Model } \\
\text { OR }(95 \% \mathrm{CI})\end{array}$ & P Value & $\begin{array}{l}\text { Multivariable model }{ }^{\mathrm{i}} \\
\text { OR }(95 \% \mathrm{Cl})\end{array}$ & P Value \\
\hline Age, year & $1.003(1.000-1.005)$ & 0.032 & $1.002(0.996-1.007)$ & 0.013 \\
\hline APACHE & $1.006(1.000-1.011)$ & 0.032 & $1.004(1.001-1.007)$ & 0.554 \\
\hline $\begin{array}{l}\text { Nonsocomial } \\
\text { acquired Infection }\end{array}$ & $1.878(1.727-2.043)$ & $\leq 0.001$ & $1.699(1.517-1.904)$ & $\leq 0.001$ \\
\hline AIDS & $0.825(0.620-1.099)$ & 0.188 & $1.066(0.789-1.439)$ & 0.677 \\
\hline Leukemia & $0.879(0.724-1.069)$ & 0.197 & $0.797(0.636-0.998)$ & 0.048 \\
\hline Metastatic Cancer & $1.196(1.044-1.371)$ & 0.010 & $1.058(0.914-1.225)$ & 0.448 \\
\hline Immunosuppression & $1.166(1.034-1.316)$ & 0.012 & $1.088(0.929-1.274)$ & 0.293 \\
\hline Organ Transplant & $1.368(1.114-1.681)$ & 0.003 & $1.184(0.938-1.496)$ & 0.155 \\
\hline Neutropenia & $1.175(0.951-1.144)$ & 0.114 & $1.135(0.888-1.451)$ & 0.312 \\
\hline Liver Failure & $1.180(1.006-1.385)$ & 0.042 & $1.227(1.028-1.463)$ & 0.023 \\
\hline Chronic Heart Failure & $0.905(0.790-1.037)$ & 0.151 & $0.874(0.756-1.010)$ & 0.069 \\
\hline $\begin{array}{l}\text { Hypertension, high } \\
\text { blood pressure } \\
\text { medication required }\end{array}$ & $0.863(0.784-0.950)$ & 0.003 & $0.863(0.777-0.958)$ & 0.006 \\
\hline Ventilator Dependent & $2.072(1.180-3.636)$ & 0.011 & $2.088(1.159-3.761)$ & 0.014 \\
\hline Chronic Renal Failure & $1.200(1.073-1.343)$ & 0.001 & $1.154(0.979-1.360)$ & 0.088 \\
\hline Chronic Dialysis & $1.237(1.065-1.437)$ & 0.005 & $1.075(0.866-1.334)$ & 0.512 \\
\hline $\begin{array}{l}\text { Diabetes Mellitus, } \\
\text { insulin dependent }\end{array}$ & $1.254(1.083-1.452)$ & 0.002 & $1.215(1.036-1.424)$ & 0.017 \\
\hline Elective Surgery & $1.643(1.472-1.834)$ & $\leq 0.001$ & $1.332(1.166-1.521)$ & $\leq 0.001$ \\
\hline Emergent Surgery & $1.538(1.314-1.800)$ & $\leq 0.001$ & $1.244(1.045-1.482)$ & 0.014 \\
\hline ETOH & $0.846(0.748-0.957)$ & 0.008 & $0.929(0.810-1.065)$ & 0.292 \\
\hline $\begin{array}{l}\text { Organic Brain, } \\
\text { dementia/multiple } \\
\text { CVA }\end{array}$ & $0.862(0.715-1.039)$ & 0.120 & $0.881(0.724-1.073)$ & 0.207 \\
\hline $\begin{array}{l}\text { Neuromuscular } \\
\text { Disease }\end{array}$ & $1.407(1.072-1.847)$ & 0.014 & $1.540(1.153-2.057)$ & 0.003 \\
\hline ER & $0.800(0.734-0.872)$ & $\leq 0.001$ & $1.166(0.926-1.468)$ & 0.191 \\
\hline Medical & $1.291(1.180-1.412)$ & $\leq 0.001$ & $1.150(0.915-1.446)$ & 0.232 \\
\hline Surgical & $1.367(1.225-1.525)$ & $\leq 0.001$ & $1.055(0.825-1.348)$ & 0.670 \\
\hline Other ER & $0.697(0.601-0.809)$ & $\leq 0.001$ & $0.999(0.766-1.303)$ & 0.994 \\
\hline Other ICU & $0.771(0.627-0.948)$ & 0.014 & $0.919(0.680-1.243)$ & 0.583 \\
\hline
\end{tabular}

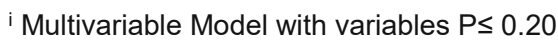


Table 2 - Multivariable Logistic Regression for Independent Predictors of ICU Mortality

\begin{tabular}{|c|c|c|c|c|c|c|c|c|}
\hline $\begin{array}{l}\text { Patient } \\
\text { Characteristic }\end{array}$ & $\begin{array}{l}\text { Univariable Model } \\
\text { with Mortality OR } \\
(95 \% \mathrm{Cl})\end{array}$ & P Value & \begin{tabular}{|l|} 
Multivariable \\
model $^{1}$ \\
OR $(95 \% \mathrm{Cl})$ \\
\end{tabular} & P Value & $\begin{array}{l}\text { Multivariable } \text { model }^{2} \\
\text { OR }(95 \% \mathrm{Cl})\end{array}$ & P Value & \begin{tabular}{|l|} 
Multivariable model $^{3}$ \\
OR $(95 \% \mathrm{Cl})$
\end{tabular} & P Value \\
\hline Age, year & $1.016(1.014,1.019)$ & $\leq 0.001$ & $1.016(1.013,1.020)$ & $\leq 0.001$ & $1.015(1.012,0.019)$ & $\leq 0.001$ & $1.016(1.012,1.019)$ & $\leq 0.001$ \\
\hline APACHE & $1.127(1.120,1.134)$ & $\leq 0.001$ & $1.121(1.113,1.128)$ & $\leq 0.001$ & $1.116(1.109,1.124)$ & $\leq 0.001$ & $1.095(1.087,1.104)$ & $\leq 0.001$ \\
\hline $\begin{array}{l}\text { Nonsocomial } \\
\text { acquired Infection }\end{array}$ & $1.597(1.469,1.723)$ & $\leq 0.001$ & $1.273(1.134)$ & $\leq 0.001$ & $1.350(1.200,1.518)$ & $\leq 0.001$ & $1.062(1.396,1.838)$ & $\leq 0.001$ \\
\hline AIDS & $1.249(0.970,1.609)$ & 0.085 & $1.911(1.410,2.591)$ & $\leq 0.001$ & $1.935(1.420,2.636)$ & $\leq 0.001$ & $1.829(1.282,2.610)$ & 0.001 \\
\hline Lymphoma & $1.836(1.49,2.264)$ & $\leq 0.001$ & $1.237(0.956,1.601)$ & 0.1069 & $1.237(0.952,1.607)$ & 0.111 & $1.293(0.955,1.749)$ & 0.096 \\
\hline Leukemia & $2.115(1.773,2.523)$ & $\leq 0.001$ & $1.132(0.902,1.420)$ & 0.284 & $1.088(0.864,1.371)$ & 0.472 & $0.951(0.727,1.244)$ & 0.712 \\
\hline Metastatic Cancer & $1.408(1.238,1.601)$ & $\leq 0.001$ & $1.297(1.115,1.507)$ & 0.001 & $1.233(1.058,1.435)$ & 0.007 & $1.180(0.989,1.408)$ & 0.067 \\
\hline $\begin{array}{l}\text { Immunosuppressi } \\
\text { on }\end{array}$ & $1.787(1.596,2.002)$ & $\leq 0.001$ & $1.263(1.082,1.475)$ & 0.003 & $1.212(1.036,1.418)$ & 0.016 & $1.157(0.963,1.389)$ & 0.120 \\
\hline Neutropenia & $2.493(2.055,3.024)$ & $\leq 0.001$ & $1.254(0.969,1.623)$ & 0.085 & $1.199(0.932,1.559)$ & 0.174 & $1.070(0.786,1.456)$ & 0.669 \\
\hline NY heart failure & $1.677(1.310,2.148)$ & $\leq 0.001$ & $1.648(1.243,2.184)$ & 0.001 & $1.679(1.260,2.238)$ & $\leq 0.001$ & $1.557(1.117,2.172)$ & 0.009 \\
\hline Liver Failure & $2.600(2.22,3.035)$ & $\leq 0.001$ & $2.515(2.103,3.008)$ & $\leq 0.001$ & $2.517(2.098,3.020)$ & $\leq 0.001$ & $2.301(1.865,2.839)$ & $\leq 0.001$ \\
\hline $\begin{array}{l}\text { Chronic Heart } \\
\text { Failure }\end{array}$ & $1137(1.004,1.287)$ & 0.043 & $1.063(0.919,1.230)$ & 0.408 & $1.027(0.886,1.191)$ & 0.720 & $0.940(0.791,1.117)$ & 0.481 \\
\hline $\begin{array}{l}\text { Hypertension } \\
\text { high blood } \\
\text { pressure } \\
\text { medication } \\
\text { required }\end{array}$ & $0.836(0.765-0.915)$ & $\leq 0.001$ & $0.836(0.750,0.932)$ & 0.001 & $0.863(0.773,0.964)$ & 0.009 & $1.116(0.984,1.267)$ & 0.089 \\
\hline $\begin{array}{l}\text { Ventilator } \\
\text { Dependent }\end{array}$ & $0.867(0.489,1.567)$ & 0.654 & $0.927(0.480,1.793)$ & 0.823 & $1.119(0.575,2.178)$ & 0.740 & $1.105(0.530,2.300)$ & 0.791 \\
\hline $\begin{array}{l}\text { Chronic } \\
\text { Obstructive } \\
\text { Pulmonary } \\
\text { Disease }\end{array}$ & $1.129(0.963,1.324)$ & 0.134 & $1.015(8.46,1.219)$ & 0.869 & $1.061(0.881,1.277)$ & 0.534 & $1.082(0.873,1.341)$ & 0.471 \\
\hline $\begin{array}{l}\text { Chronic Renal } \\
\text { Failure }\end{array}$ & $1.073(0.964,1.194)$ & 0.198 & $0.807(0.710,0.918)$ & 0.001 & $0.794(0.697,0.904)$ & 0.001 & $0.833(0.716,0.968)$ & 0.017 \\
\hline $\begin{array}{l}\text { Diabetes, } \\
\text { Medication }\end{array}$ & $0.861(0.781,0.949)$ & 0.003 & $0.889(0.790,0.999)$ & 0.048 & $0.874(0.777,0.985)$ & 0.027 & $0.888(0.774,1.019)$ & 0.090 \\
\hline $\begin{array}{l}\text { Diabetes, Insulin } \\
\text { Dep }\end{array}$ & $0.833(0.721,0.963)$ & 0.013 & $0.730(0.613,0.869)$ & $\leq 0.001$ & $0.732(0.614,0.872)$ & 0.001 & $0.745(0.608,0.912)$ & 0.004 \\
\hline IVDU & $0.487(0.352,0.674)$ & $\leq 0.001$ & $0.651(0.438,0.967)$ & 0.034 & $0.678(0.455,1.010)$ & 0.056 & $0.703(0.453,1.090)$ & 0.115 \\
\hline Thromboembolitc & $0.766(0.549,1.069)$ & 0.117 & $0.836(0.571,1.226)$ & 0.360 & $0.806(0.547,1.186)$ & 0.273 & $0.876(0.557,1.376)$ & 0.565 \\
\hline $\begin{array}{l}\text { Neuromuscular } \\
\text { Disease }\end{array}$ & $0.573(0.428,0.769)$ & $\leq 0.001$ & $0.930(0.666,1.298)$ & 0.670 & $0.935(0.669,1.305)$ & 0.692 & $0.883(0.604,1.289)$ & 0.519 \\
\hline Elective Surgery & $0.900(0.807,1.004)$ & 0.058 & $0.868(0.753,1.001)$ & 0.052 & $0.888(0.769,1.025)$ & 0.105 & $1.021(0.864,1.207)$ & 0.805 \\
\hline Any resistance & $1.063(0.978,1.156)$ & 0.151 & $0.963(0.873,1.062)$ & 0.451 & $0.970(0.879,1.072)$ & 0.554 & $1.004(0.894,1.127)$ & 0.950 \\
\hline \multicolumn{9}{|l|}{ Admission Source } \\
\hline ER & $0.745(0.688,0.807)$ & $\leq 0.0010$ & $0.743(0.596,0.927)$ & 0.008 & $0.657(0.525,0.823)$ & $\leq 0.001$ & $0.567(0.439,0.732)$ & $\leq 0.001$ \\
\hline Medical & $1.66(1.530,1.813)$ & $\leq 0.001$ & $1.045(0.834,1.309)$ & 0.702 & $0.955(0.759,1.201)$ & 0.692 & $1.000(0.771,1.298)$ & 0.999 \\
\hline Surgical & $0.932(0.837,1.037)$ & 0.195 & $0.846(0.663,1.078)$ & 0.0176 & $0.775(0.605,0.991)$ & 0.043 & $0.897(0.663,1.166)$ & 0.372 \\
\hline Other ER & $0.657(0.573,0.754)$ & $\leq 0.001$ & $0.685(0.530,0.885)$ & 0.004 & $0.609(0.470,0.790)$ & $\leq 0.001$ & $0.499(0.371,0.670)$ & $\leq 0.001$ \\
\hline Other Ward & $1.167(0.965,1.412)$ & 0.111 & $0.864(0.640,1.166)$ & 0.339 & $0.803(0.592,1.089)$ & 0.157 & $0.803(0.569,1.133)$ & 0.212 \\
\hline LN ICU LOS & $0.629(0.602,0.657)$ & $\leq 0.001$ & & & $0.677(0.643,0.712)$ & $\leq 0.001$ & & \\
\hline LN Hosp LOS & $0.277(0.263,0.291)$ & $\leq 0.001$ & & & & & $0.278(0.262,0.295)$ & $\leq 0.001$ \\
\hline
\end{tabular}

1 Multivariable Model $\mathrm{P}<0.20$ fit with resistance

2 Multivariable Model $\mathrm{P}<0.20$ fit with resistance and LN ICU LOS

3 Multivariable Model $\mathrm{P}<0.20$ fit with resistance and LN HOSP LOS 
Table 3 - Multivariable Logistic Regression for Independent Predictors of Hospital Mortality

\begin{tabular}{|c|c|c|c|c|c|c|c|c|}
\hline $\begin{array}{l}\text { Patient } \\
\text { Characteristic }\end{array}$ & $\begin{array}{l}\text { Univariable Model with } \\
\text { Mortality OR }(95 \% \mathrm{CI})\end{array}$ & $\begin{array}{l}\mathrm{P} \\
\text { Value }\end{array}$ & $\begin{array}{l}\text { Multivariable } \\
\text { model}^{1} \text { OR }(95 \% \mathrm{CI})\end{array}$ & \begin{tabular}{|l}
$P$ \\
Value
\end{tabular} & $\begin{array}{l}\text { Multivariable } \\
\text { model }^{2} \\
\text { OR }(95 \% \mathrm{CI})\end{array}$ & $\begin{array}{l}\mathbf{P} \\
\text { Value }\end{array}$ & \begin{tabular}{l|} 
Multivariable \\
model ${ }^{3}$ OR $(95 \% \mathrm{Cl})$
\end{tabular} & $\begin{array}{l}\mathbf{P} \\
\text { Value }\end{array}$ \\
\hline Age, year & $1.023(1.020-1.025)$ & $\leq 0.001$ & $1.025(1.022,1.029)$ & $\leq 0.001$ & $1.025(1.021,1.028)$ & $\leq 0.001$ & $1.026(1.123,1.030)$ & $\leq 0.001$ \\
\hline APACHE & $1.127(1.120-1.134)$ & $\leq 0.001$ & $1.119(1.111,1.126)$ & $\leq 0.001$ & $1.116(1.109,1.124)$ & $\leq 0.001$ & $1.099(1.091,1.107)$ & $\leq 0.001$ \\
\hline $\begin{array}{l}\text { Nonsocomial } \\
\text { acquired } \\
\text { Infection }\end{array}$ & $2.082(1.923-2.255)$ & $\leq 0.001$ & $1.563(1.393,1.754)$ & $\leq 0.001$ & $0.324(1.446,1.824)$ & $\leq 0.001$ & $2.040(1.790,2.325)$ & $\leq 0.001$ \\
\hline AIDS & $1.208(0.938-1.555)$ & 0.142 & $2.362(1.737,3.212)$ & $\leq 0.001$ & $2.384(1.750,3.247)$ & $\leq 0.001$ & $2.318(1.650,3.257)$ & $\leq 0.001$ \\
\hline Lymphoma & $2.236(1.789,2.794)$ & $\leq 0.001$ & $1.832(1.393,2.409)$ & $\leq 0.001$ & $1.834(1.394,2.414)$ & $\leq 0.001$ & $1.987(1.469,2.687)$ & $\leq 0.001$ \\
\hline Leukemia & $2.430(2.012-2.935)$ & $\leq 0.001$ & $1.445(1.135,1.839)$ & 0.003 & $1.413(1.108,1.802)$ & 0.005 & $1.271(0.969,1.668)$ & 0.083 \\
\hline $\begin{array}{l}\text { Metastatic } \\
\text { Cancer }\end{array}$ & $1.537(1.351-1.751)$ & $\leq 0.001$ & $1.436(1.232,1.675)$ & $\leq 0.001$ & $1.387(1.189,1.619)$ & $\leq 0.001$ & $1.363(1.149,1.617)$ & $\leq 0.001$ \\
\hline $\begin{array}{l}\text { Immunosuppres } \\
\text { sion }\end{array}$ & $1.734(1.545-1.946)$ & $\leq 0.001$ & $1.118(0.954,1.310)$ & 0.168 & $1.088(0.928,1.276)$ & 0.299 & $1.010(0.845,1.208)$ & 0.912 \\
\hline Neutropenia & $2.613(2.125-3.213)$ & $\leq 0.001$ & $1.286(0.977,1.693)$ & 0.073 & $1.243(0.942,1.640)$ & 0.124 & $1.089(0.797,1.488)$ & 0.591 \\
\hline Liver Failure & $3.117(2.630-3.694)$ & $\leq 0.001$ & $3.498(2.875,4.257)$ & $\leq 0.001$ & $3.510(2.879,4280)$ & $\leq 0.001$ & $3.359(2.697,4.184)$ & $\leq 0.001$ \\
\hline $\begin{array}{l}\text { New York Heart } \\
\text { Failure }\end{array}$ & $1.940(1.498,2.513)$ & $\leq 0.001$ & $1.555(1.154,2.093)$ & 0.004 & $1.573(1.166,2.123)$ & 0.003 & $1.444(1.035,2.015)$ & 0.031 \\
\hline $\begin{array}{l}\text { Chronic Heart } \\
\text { Failure }\end{array}$ & $1.249(1.104-1.413)$ & $\leq 0.001$ & $1.097(0.946,1.272)$ & 0.221 & $1.073(0.925,1.245)$ & 0.353 & $1.022(0.865,1.207)$ & 0.800 \\
\hline Intermittent HD & $1.135(1.012,1.272)$ & 0.030 & $1.094(0.952,1.256)$ & 0.204 & $1.095(0.953,1.258)$ & 0.200 & $1.073(0.921,1.250)$ & 0.364 \\
\hline $\begin{array}{l}\text { Hypertension } \\
\text { high blood } \\
\text { pressure } \\
\text { medication } \\
\text { required }\end{array}$ & $0.766(0.702-0.836)$ & $\leq 0.001$ & $0.690(0.619,0.769)$ & $\leq 0.001$ & $0.702(0.630,0.783)$ & $\leq 0.001$ & $0.816(0.723,0.921)$ & 0.001 \\
\hline Severe COPD & $1.301(1.11,1.524)$ & 0.001 & $1.144(0.952,1.375)$ & 0.151 & $1.183(0.983,1.423)$ & 0.076 & $1.226(0.999,1.504)$ & 0.051 \\
\hline $\begin{array}{l}\text { Ventilator } \\
\text { Dependent }\end{array}$ & $0.905(0.516-1.588)$ & 0.728 & $0.920(0.477,1.772)$ & 0.802 & $1.035(0.536,1.998)$ & 0.918 & $1.012(0.499,2.054)$ & 0.973 \\
\hline $\begin{array}{l}\text { Chronic Renal } \\
\text { Failure }\end{array}$ & $1.277(1.149-1.419)$ & $\leq 0.001$ & $0.857(0.722,1.017)$ & 0.078 & $0.846(0.712,1.004)$ & 0.056 & $0.861(0.710,1.042)$ & 0.125 \\
\hline Chronic Dialysis & $1.265(1.097-1.459)$ & 0.001 & $1.248(0.994,1.566)$ & 0.56 & $1.247(0.993,1.565)$ & 0.057 & $1.395(1.085,1.793)$ & 0.010 \\
\hline \begin{tabular}{l|} 
Diabetes, \\
Medication \\
Required \\
\end{tabular} & $0.867(0.789,0.953)$ & 0.003 & $0.933(0.832,1.047)$ & 0.240 & $0.825(0.824,1.038)$ & 0.183 & $0.947(0.833,1.076)$ & 0.402 \\
\hline $\begin{array}{l}\text { Emergent } \\
\text { Surgery }\end{array}$ & $1.227(1.052-1.430)$ & 0.009 & $1.118(0.929,1.346)$ & 0.237 & $1.167(0.968,1.406)$ & 0.105 & $1.339(1.087,1.649)$ & 0.006 \\
\hline IV Drug Use & $0.425(0.313,0.577)$ & $\leq 0.001$ & $0.648(0.440,0.955)$ & 0.028 & $0.665(0.451,0.981)$ & 0.040 & $0.674(0.446,1.019)$ & 0.062 \\
\hline $\begin{array}{l}\text { Organic Brain, } \\
\text { dementia }\end{array}$ & $1.167(0.988-1.379)$ & 0.069 & $0.998(0.821,1.213)$ & 0.985 & $0.988(0.812,1.202)$ & 0.904 & $1.075(0.864,1.337)$ & 0.515 \\
\hline $\begin{array}{l}\text { Neuromuscular } \\
\text { Disease }\end{array}$ & $0.561(0.427-0.738)$ & $\leq 0.001$ & $0974(0.705,1.346)$ & 0.873 & $0.975(0.706,1.346)$ & 0.877 & $0.937(0.657,1.337)$ & 0.719 \\
\hline \multicolumn{9}{|l|}{$\begin{array}{l}\text { Admission } \\
\text { Source }\end{array}$} \\
\hline ER & $0.667(0.617-0.721)$ & $\leq 0.001$ & $0.923(0.802,1.062)$ & 0.265 & $0.901(0.783,1.038)$ & 0.149 & $0.729(0.622,0.854)$ & $\leq 0.001$ \\
\hline Medical & $2.095(1.922-2.283)$ & $\leq 0.001$ & $1.492(1.305,1.705)$ & $\leq 0.001$ & $1.485(1.298,1.699)$ & $\leq 0.001$ & $1.439(1.239,1.672)$ & $\leq 0.001$ \\
\hline Other ER & $0.524(0.458-0.598)$ & $\leq 1.00$ & $0.741(0.613,0.897)$ & 0.002 & $0.723(0.597,0.876)$ & 0.001 & $0.539(0.435,0.667)$ & $\leq 0.001$ \\
\hline Other ICU & $0.884(0.738-1.059)$ & 0.181 & $1.048(0.830,1.324)$ & 0.691 & $1.102(0.871,1.393)$ & 0.419 & $0.961(0.742,1.246)$ & 0.766 \\
\hline Resistance Any & $1.214(1.118-1.318)$ & $\leq 0.001$ & $1.055(0.957,1.164)$ & 0.282 & $1.062(0.962,1.172)$ & 0.232 & $1.111(0.996,1.240)$ & 0.059 \\
\hline LN ICU LOS & $0.734(0.704-0.765)$ & $\leq 0.001$ & & & $0.780(0.742,0.820)$ & $\leq 0.001$ & & \\
\hline LN Hosp LOS & $0.378(0.363-0.394)$ & $\leq 0.001$ & & & & & $0.354(0.335,0.373)$ & $\leq 0.001$ \\
\hline
\end{tabular}

1 Multivariable Model $\mathrm{P}<0.20$ fit with resistance

2 Multivariable Model $\mathrm{P}<0.20$ fit with resistance and LN ICU LOS

3 Multivariable Model $P<0.20$ fit with resistance and LN HOSP LOS 


\section{Epidemiology of death in a single PICU over 15 years}

Sloof, Natalie $^{1}$; Miller, Michael R. ${ }^{1,2}$; Sarpal, Amrita ${ }^{1,2,3}$

1 Schulich School of Medicine \& Dentistry, Western University, London, Ontario, Canada

2 Children's Health Research Institute, London, Ontario, Canada

3 Paediatric Critical Care Medicine, Children's Hospital London Health Sciences Centre, London, Ontario, Canada

Introduction/Background: Advances in medicine have significantly reduced but not eliminated pediatric mortality. The majority of pediatric deaths occur within pediatric or neonatal intensive care units following withdrawal of life sustaining treatment (WLST), brain death (BD), failed cardiopulmonary resuscitation (CPR), or limitation of treatment (LT). The increasing availability of pediatric palliative care, provincial legislative changes regarding withholding and withdrawal of life-sustaining treatment, and increasing long-term survival of pediatric patients with chronic, critical illness may influence the distribution of modes of death.

Objectives: To describe mode of death and changes over time as they relate to patient mortality over a 15 -year period in a single Canadian pediatric intensive care unit (PICU).

Methods: A retrospective chart review was completed for all patients who died in the PICU at Children's Hospital - London Health Sciences Centre from January 1, 2003 to December 31, 2017. Demographic data (including sex, age, chronic conditions, reliance on medical technology), admission details (including admission diagnosis, admission source, PELOD severity score, length of stay), and factors surrounding death (including cause of death, mode of death, organ donation, do not resuscitate orders) were collected. Over the 15-year period, 326 patients were identified. Mortality by year ranged from $1.1 \%$ to $4.6 \%$, with a median of $3.2 \%$. Preliminary results showed overall proportions of mode of death were $59.5 \%$ WLST, $13.2 \%$ BD, $15.0 \%$ failed CPR, and $12.3 \% \mathrm{LT}$; these trends were fairly consistent over the 15-year period. The three most common causes of death were injury/poisoning (24.8\%), infection (13.8\%), and congenital $(11.0 \%)$. Twenty percent of the patients who died in the PICU were dependent on technology prior to PICU admission. Approximately $50 \%$ had a significant premorbid condition. Further analyses are pending.

Conclusions: Preliminary analyses indicated that WLST was the most common mode of death. Of the patients in the PICU who died, the proportion that had existing dependence on medical technology has not changed over time. Further investigations with larger patient populations are necessary to elucidate trends in mode of death over time.

\section{References}

1. Burns KH, Casey PH, Lyle RE, Mac Bird T, Fussell JJ, Robbins JM. Increasing prevalence of medically complex children in US hospitals. Pediatrics. 2010;126(4):638-646.

2. de Vos MA, Bos AP, Plötz FB, et al. Talking with parents about end-of-life decisions for their children. Pediatrics. 2015:peds. 2014-1903.

3. Garros D, Rosychuk RJ, Cox PN. Circumstances surrounding end of life in a pediatric intensive care unit. Pediatrics. 2003;112(5):e371-e371.

4. Sands R, Manning JC, Vyas $\mathrm{H}$, Rashid A. Characteristics of deaths in paediatric intensive care: a 10-year study. Nursing in critical care. 2009;14(5):235-240.

5. Behrman RE, Field MJ. When children die: Improving palliative and end-of-life care for children and their families. National Academies Press; 2003.

6. Widger K, Davies D, Rapoport A, et al. Pediatric palliative care in Canada in 2012: a cross-sectional descriptive study. CMAJ open. 2016;4(4):E562. 
7. College of Physicians and Surgeons of Ontario. Planning for and providing quality end of life care, Policy Statement \#4-15, September 2015. Available from URL: http://www.cpso.on.ca/CPSO/media/documents/Policies/Policy-Items/End-ofLife.pdf?ext=.pdf (accessed April 2018). 


\section{Establishing Feasibility \& Safety of Providing Critical Care for Patients with Ebola through Design of a Simulated Ebola Treatment Unit}

Kiiza, Peter ${ }^{1}$; Mullin, Sarah ${ }^{1}$; Teo, Koren ${ }^{2}$; Perez, Adic ${ }^{1}$; Piquette, Dominique ${ }^{3}$; Lee, James $^{3}$; Thompson, Kelly ; Kao, Raymond ${ }^{5}$; Chan, Adrienne ${ }^{6}$; Mishra, Sharmistha ${ }^{6}$; Hajek, Jan; Murthy, Srinivas ${ }^{7}$; Lamontagne, Francois ${ }^{8}$; Tien, Homer ${ }^{9}$; Marshall, John; Simor, Andy ${ }^{6}$; Burgess, Rob ${ }^{10}$; Hall, Trevor ${ }^{10}$; Christian, Michael ${ }^{11}$; Pinto, Ruxandra ${ }^{1}$; Vanderschuren, Abel'12; Goodman, Len ${ }^{13}$; Adhikari, Neill'; Fowler, Rob ${ }^{3}$

1 Sunnybrook Research Institute, Sunnybrook Health Sciences Centre, Toronto, Canada

2 Canadian Forces Health Services Group, Toronto, Ontario, Canada

3 Interdepartmental Division of Critical Care Medicine, University of Toronto, Toronto, Ontario, Canada

4 The George Institute for Global Health, Sydney, Australia

5 Chief of Medicine, Canadian Forces Health Services, Associate Professor, Western University, London, Ontario, Canada

6 Division of Infectious Diseases, Department of Medicine, University of Toronto, Toronto, Ontario, Canada

7 Division of Infectious Diseases, Department of Medicine, University of British

Columbia, Vancouver, British Columbia, Canada

8 Centre hospitalier universitaire de Sherbrooke, Quebec, Canada

9 Department of Surgery, University of Toronto, Toronto, Ontario, Canada

10 Sunnybrook Hospital, Toronto, Ontario, Canada

11 Clinical Lead (Safety, Education \& Development) at Essex \& Herts Air Ambulance (EHAAT), London, United Kingdom

12 Centre hospitalier universitaire de Québec, Quebec City, Quebec, Canada 13 Scientist, Defence Research and Development Canada, Toronto, Ontario, Canada

Introduction: The provision of advanced medical care was not routinely available in the 2014-2016 West Africa outbreak of Ebola Virus Disease (EVD). As care became more organized and medical interventions increased, case-fatality rate dropped from $70 \%$ to $40 \%$. Understanding the feasibility of and barriers to the application of advanced organ supportive care for such patients is important for care in future outbreaks.

Objective: To explore the feasibility and safety of delivering advanced medical care to patients in a simulated Ebola treatment unit. The objective of the pilot study was to develop a protocol of simulated advanced supportive care under two climatic conditions: 'hot'(35 0 C, 60\% humidity); 'cool' (20 0 C, 20\% humidity).

Methods: Participants were healthcare workers; they received an information package and instructional videos of the study procedures and completed a pre-study medical fitness checklist. Study procedures included donning and doffing of personal protective equipment (PPE), inserting a peripheral venous catheter (PIV) and a mid-line arm venous catheter (mid-line catheter), and endotracheal intubation. Participants were assigned to either hot or cool conditions and performed tasks on mannequins. Heart rate $(H R)$, respiratory rate $(R R)$, skin temperature, and blood pressure $(B P)$ monitors were attached to each participant. Weight, height, and baseline and post-simulation vital signs were measured. Task feasibility was measured using a task specific checklist, participants' task completion times, and percentages of tasks completed. Safety was assessed by vital signs measurements, number of PPE breaches, and health-concerning and near-miss incidents. Health-concerning threshold values for simulation cessation were $\mathrm{HR}>85 \%$ of maximum $\mathrm{HR}$ or $<40$ beats/minute, systolic BP $<90 \mathrm{mmHg}$ or a drop $>40 \mathrm{mmHg}$ below baseline, a self-rated thermal comfort scale of $\geq 12$ (from 1, so cold that I am helpless to 13 , so hot I am sick and nauseated), or any report of feeling unwell.

Results: 14 healthcare workers participated, 3 others were excluded after a medical assessment. There were 9 physicians and 5 nurses, $50 \%$ were female. Mean (SD) age 
was 41 (7) years and BMI was 24.8 (6.4). 9 participants had prior experience of working in austere environments. 13 participants completed all simulation tasks with 67 (6.8) minutes in the chamber. Mean task completion times were 13.1 (3.0) min for PIV, 33.9 (3.5) min for mid-line catheter insertion and 15.5 (4.4) min for intubation. Mean task completion percentages were: 72.7 (7.1) \% for PIV, 75.5 (18.4) \% for mid-line catheter insertion, 70.4 (22.8) \% for intubation. 4 nurses provided feedback on lack of feasibility of mid-line catheter insertion and intubation. Mean hot and cool condition temperatures were 31.5 (2.2) $0 \mathrm{C}$ and 20.2 (0.25) $0 \mathrm{C}$ (chamber), mean skin and tympanic temperatures were; $34.2(1.3) 0 \mathrm{C}$ and $37.2(0.2) 0 \mathrm{C}$ respectively. There were 6 health concerning-incidents in 4 participants (all allocated to the hot condition); 42 minor breaches in PPE use, with at least 1 breach in each participant; and 37 nearmiss incidents in 13 participants. We terminated the simulation of 1 participant who was nauseous and heat strained. All participants had near baseline vital signs measurements at the end of their simulation.

Conclusion: For physicians, the procedural tasks appear feasible. The study team is currently developing tasks and case scenarios specifically tailored to nurses. In the full study, participants will be randomized to two temperature/humidity conditions.

Table 1 - Demographics and baseline characteristics

\begin{tabular}{|l|l|}
\hline Characteristics & N=14 participants \\
\hline Age in years, mean (SD) & $41.4(7.1)$ \\
\hline BMI, mean (SD) & $24.8(6.4)$ \\
$\mathrm{n}(\%)$ & $8(57.1)$ \\
$<25$ & $5(35.7)$ \\
$25-29.9$ & $1(7.1)$ \\
$\geq 30$ & $7(50)$ \\
\hline Sex Female, $\mathrm{n}(\%)$ & \\
\hline Profession, $\mathrm{n}(\%)$ & $5(36.7)$ \\
Nurse & $9(64.3)$ \\
Physician & \\
\hline Department $\mathrm{n}(\%)$ & $9(64.3)$ \\
Critical care & $3(21.4)$ \\
Emergency & $2(14.3)$ \\
Other in-patient care & \\
\hline Working Experience, in years $\mathrm{n}(\%)$ & $4(28.6)$ \\
<10 & $6(42.8)$ \\
10-20 & $4(28.6)$ \\
$>20$ & $2(24.3)$ \\
\hline Work experience in the ETU, $\mathrm{n}(\%)$ & $9(64.3)$ \\
Work experience in austere environment, $\mathrm{n}(\%)$ & \\
\hline Prior procedure experience, $\mathrm{n}(\%)$ & $12(85.7)$ \\
PIV & $3(21.4)$ \\
Mid-line catheter & $10(71.4)$ \\
CVC & $10(71.4)$ \\
Endotracheal Intubation & $2(14.3)$ \\
Triple packaging of blood samples &
\end{tabular}


Figure 1 - Boxplots showing median percentage ( $1^{\text {st }}$ and $3^{\text {rd }}$ quartiles) of completed tasks

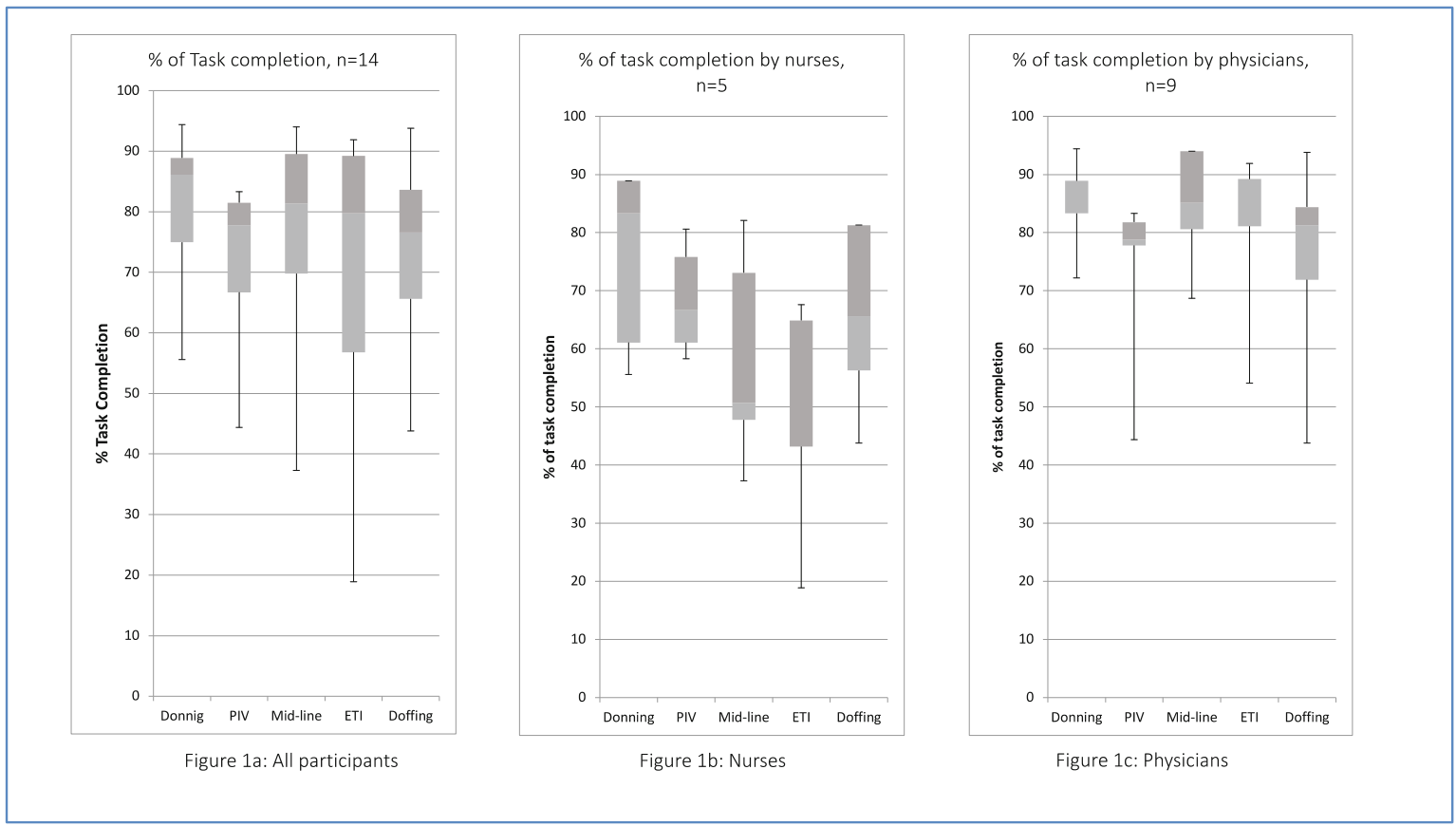

Figure 2 - Changes in SBP: Systolic Blood Pressure, DBP: Diastolic Blood Pressure and HR: Heart Rate throughout the simulation

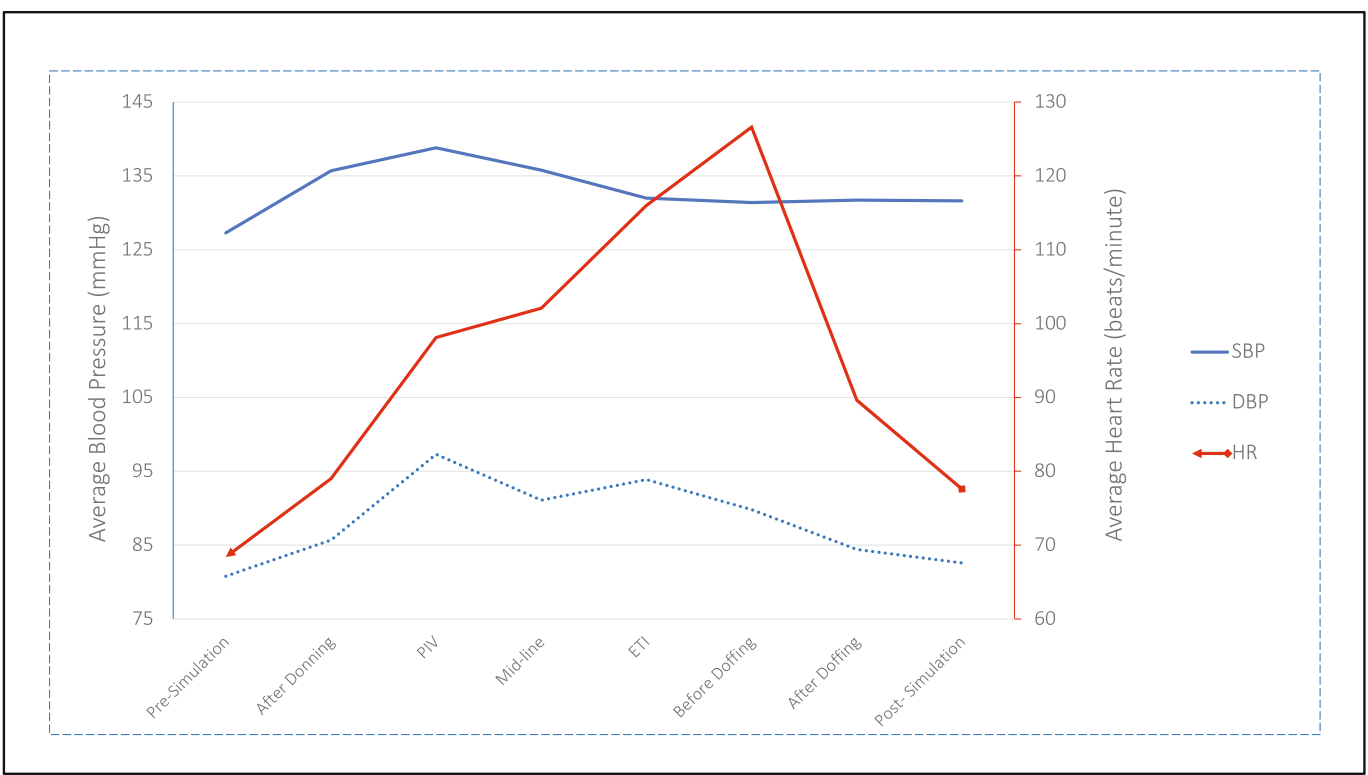




\section{References}

1. Lamontagne F, Fowler RA, Adhikari NK, Murthy S, Brett-Major DM, Jacobs M, Uyeki TM, Vallenas C, Norris SL, Fischer 2nd WA, Fletcher TE. Evidence-based

guidelines for supportive care of patients with Ebola virus disease. The Lancet. 2018 Feb 17;391(10121):700-8. 2.

2. World Health Organization. Situation Reports. Available at:

http://apps.who.int/ebola/ebola-situation-reports. Accessed July 20, 2018. 
Evidence Bulletins: Bringing Evidence-Based Recommendations on Donor Management to the Bedside

Arora, SA ${ }^{1,2,3}$; Akhtar, $\mathrm{A}^{4,5}$; Rochwerg, B3,6; D'Agostino, $\mathrm{K}^{8}$; Frenette, AJ ${ }^{9,10}$; D'Aragon, $\mathrm{F}^{11,12 ;}$ Belley-Cote, $\mathrm{E}^{13,14,15,16}$; Meade, $\mathrm{MO}^{3,6}$

1 Division of Critical Care, Department of Medicine, Northern Ontario School of

Medicine, Thunder Bay, Canada

2 Division of Critical Care, Department of Medicine, Mackenzie Health, Toronto, Canada

3 Department of Health Research Methods, Evidence, and Impact, McMaster University, Hamilton, Canada

4 The Department of Mental Health and Substance Abuse, World Health Organization, Geneva, Switzerland

5 University of New South Wales, Sydney, Australia

6 Division of Critical Care, Department of Medicine, McMaster University, Hamilton,

Canada

7 Department of Health Research Methods, Evidence, and Impact, McMaster

University, Hamilton, Canada

8 Doblin-Deloitte Inc.

9 Pharmacy Faculty, Université de Montreal, Montreal, Quebec, Canada

10 Hopital Sacre-Coeur de Montreal, Montreal, Quebec, Canada

11 Department of Anesthesiology, Université de Sherbrooke, Sherbrooke, Quebec, Canada

12 Centre de Recherche du Centre Hospitalier Universitaire de Sherbrooke,

Sherbrooke, Quebec, Canada

13 Department of Clinical Epidemiology and Biostatistics, McMaster University,

Hamilton, Ontario, Canada

14 Department of Medicine, McMaster University, Hamilton, Ontario, Canada

15 Population Health Research Institute, Hamilton, Ontario, Canada

16 Department of Medicine, Université de Sherbrooke, Sherbrooke, Quebec, Canada

Introduction: The need for donor organs exceeds supply. Nationally, 4,492 people were on Canada's organ transplant waitlists in 2016. Unfortunately, 260 patients on waitlists that same year died before receiving transplants. The majority of organ donors are deceased donors, donating through either neurologically deceased donation or controlled donation after cardiac death. Since both of these donation categories involve life-sustaining therapy, deceased donor management is primarily the purview of the intensive care clinician. In 2016, Canada had a deceased donation rate of 20.1 per million population, ranking 19th among 68 countries. Multiple organizations, including the World Health Organization, have highlighted the need for research to advance donor management in order to increase supply. In addition to the need for further research, however, there is also a paucity of tools to help clinicians provide optimal donor care. Such tools could have a significant impact on donation rates, since there is currently significant variability in how donors are managed across the country. This is despite research suggesting that best practice in the management of potential donors increases organ yield per donor.

Objectives: The objective of this knowledge translation initiative was to develop attractive, user-friendly, bedside tools to educate ICU clinicians about current Canadian practices and recommendations in deceased donor care.

Methods: We identified topics of interest through focused groups, and a selfadministered survey of members attending a 2016 scientific meeting of the Canadian Critical Care Trials Group (CCCTG). Following this, we performed dedicated literature searches and systematic reviews. We utilized Grading of Recommendations, Assessment, Development, and Evaluation (GRADE) for assessing the quality of the evidence. Recommendations arrived at using this GRADE-focused approach were 
cross-referenced to the most recently published Canadian recommendations for deceased donor care (2006). Each one-page Evidence Bulletin (EB) addresses a single treatment in the management of deceased organ donors. Designed in collaboration with a communication designer, each EB summarizes (1) current recommendations and (2) the evidence underlying these recommendations, (3) highlights tips for clinical applications, and (4) provides data on current practices in 34 Canadian hospitals, as recorded in an observational study of the Canada DONATE Research Program. Future steps for this knowledge translation initiative will include formal needs assessments and feedback through bedside testing, as well as through surveying of the CCCTG and provincial donation experts.

Results: Sample evidence bulletins are attached as electronic copies and cover 5 of 10 selected topics: corticosteroid therapy, thyroid hormone supplementation, high-dose heparin administration, lung protective mechanical ventilation, and vasopressin therapy. Their final form is as a set of laminated cards bound together by a ring at the top left corner, for easy referral at the bedside while caring for deceased organ donors. Conclusions: Evidence Bulletins are a new and potentially useful tool for distilling complex clinical information for rapid uptake and application at the bedside.

\section{References}

1. Canadian Blood Services [Internet\}. Ottawa: CBS; c2016 [cited 2018-07-02]. Organ Donation and Transplantation in Canada: 2016 System Progress Report Update. Available from: https://professionaleducation.blood.ca/sites/msi/files/cbs_odt_system_progress_rep ort_2016_update_draft_not_for_distribution_0.pdf

2. Trillium Gift of Life Network [Internet]. Toronto: TGLN; c2018 [cited 2018-07-01]. Statistics: Waiting List. Available from: https://www.giftoflife.on.ca/en/publicreporting.htm\#waitinglist3yrhistory-cal

3. Norris S. Organ Donation and Transplantation in Canada. Ottawa: Parliamentary Information and Research Service; 2018.

4. World Health Organization. International Guidelines for the Determination of DeathPhase 1 [Internet]. Canadian Blood Services; 2012 [cited 2018-07-01]. Available from: http://www.who.int/patientsafety/montreal-forum-report.pdf

5. Malinoski DJ, Daly MC, Patel MS, Oley-Graybill C, Foster III CE, Salim A. Achieving donor management goals before deceased donor procurement is associated with more organs transplanted per donor. Journal of Trauma and Acute Care Surgery. 2011 Oct 1;71(4):990-6. 
Factors Impacting Physician Recommendation for Tracheostomy in Pediatric Prolonged Mechanical Ventilation: A National Cross-Sectional Survey.

Meyer-Macaulay, Colin ${ }^{1}$; McNally, Dayre ${ }^{2}$; Katz, Sherri; Vaccani, JP3; Thebaud, Bernard $^{2}$; Harrison, Mary-Anne ${ }^{4}$; Barrowman, Nick'; Jouvet, Philippe ${ }^{5}$

1 Fellow, Critical Care Medicine, Faculty of Medicine, University of Ottawa, Ottawa, Ontario, Canada

2 Department of pediatrics, University of Ottawa, Ottawa, Ontario, Canada

3 Department of Surgery, University of Ottawa, Ottawa, Ontario, Canada

4 Children's Hospital of Eastern Ontario Research Institute, Ottawa, Ontario, Canada

5 Department of Pediatrics, Université de Montréal, Montreal, Quebec, Canada

Introduction: Recently, a consensus definition for pediatric prolonged mechanical ventilation (PMV) has been proposed, including all children or infants $>37$ weeks gestational age (GA) ventilated invasively or non-invasively $\geq 3 \mathrm{wks}^{1}$. Early tracheostomy has been shown to be associated with improved morbidity and mortality in adults, however little is known about the attitudes of physicians towards tracheostomy for pediatric $\mathrm{PMV}^{2}$.

Objectives: Characterize the stated practice of qualified Canadian physicians (pediatric intensivists, neonatologists, respirologists and otolaryngologists to tracheostomy, specifically for pediatric PMV.

Methods: We designed a 2-part cross-sectional, web-based survey for REDCap according to established methodology ${ }^{3,4}$. Part 1 consisted of questions related to demographics while part 2 presented 3 case-based scenarios followed by a series of alterations in a single relevant clinical variable. Case 1 was a neonate with bronchopulmonary dysplasia now at 40 weeks gestational age. Cases 2 and 3 were previously healthy 1 and 10-year-olds with acute respiratory distress syndrome ventilated for 3 weeks. The Chi-square test was used to compare differences in likelihood of recommending tracheostomy at 3 weeks of MV between cases and by subspecialty. The McNemar's test was used to evaluate whether altering specific clinical variables changed physician willingness to recommend tracheostomy, and its impact on preferred timing ( $\leq 3$ weeks or $>3$ weeks of MV).

Results: Overall response rate was 165/396 (42\%). We received at least 1 response all 16 Canadian tertiary-care academic hospitals performing pediatric tracheostomy. Table 1 shows that $47 / 121$ (38.8\%), 23/93 (24.7\%) and 40/87 (46.0\%) of respondents would recommend tracheostomy at or before 3 weeks of MV for case 1, 2 and 3, at baseline respectively; the difference between case 2 and 3 was statistically significant $(p<0.001)$. As shown in Figure 1 only two of 16 clinical variables - subglottic stenosis (OR 6.5 95\% Cl 1.47-59.33), and bilateral vocal paralysis (OR 5.33, 95\% Cl 1.5328.56) - significantly increased the likelihood of ever recommending tracheostomy, in case 1 , and none in cases 2 and 3 . Far more variables were associated with a decreased likelihood of ever recommending tracheostomy for all 3 cases, the strongest of which was with life limiting condition (Case 1, OR 0.06, 95\% Cl 0.02-0.17, Case 2 OR 0.03 95\% Cl 0.0-0.1, Case 3 OR 0.05 95\% Cl 0.01-0.2). The association between variables and preferred timing of tracheostomy can be seen in figure 2 . Again, in case 1 a strong association was found between recommendation for earlier tracheostomy and subglottic stenosis (OR 31 95\% CI 5.7-1263.43) or bilateral vocal cord paralysis (OR 16, 95\% Cl 4.08-137.79).

Conclusion: A significant majority of physicians appear reluctant to recommend tracheostomy for children requiring PMV. Further, there were only a small number of factors that increased the likelihood of recommending tracheostomy at $\leq 3$ weeks of MV. The information obtained in this survey will help to inform a Canada-wide prospective cohort study to evaluate the effects of early tracheostomy on patient outcomes in paediatric PMV. 
Table 1 - Likelihood of recommending tracheostomy for each case at baseline at or before 3 weeks, stratiXied by subspecialty

\begin{tabular}{|c|c|c|c|}
\hline Variable & $\begin{array}{l}\text { Case } 1 \\
\text { (Neonate w } \\
\text { BPD) }\end{array}$ & $\begin{array}{l}\text { Case } 2 \\
\text { (1 YO w } \\
\text { ARDS) }\end{array}$ & $\begin{array}{l}\text { Case } 3 \\
(10 \text { YO w } \\
\text { ARDS) }\end{array}$ \\
\hline Proportion who felt they had expertise & $\underset{m m=11}{122 / 154}(79.2)$ & $\underset{\mathrm{m}=20}{94 / 136}(69.1)$ & $\underset{\mathrm{m}=38}{87 / 129}(67.4)$ \\
\hline $\begin{array}{l}\text { Proportion of those who would recommend } \\
\text { tracheostomy at } 3 \text { weeks }\end{array}$ & $47 / 121(38.8)_{m=1}^{m}$ & $23 / 93(24.7)$ & $40 / 87(46.0)$ \\
\hline \multicolumn{4}{|c|}{ P-values* for comparison between cases of overall recommendation } \\
\hline Case 1 & - & 0.43 & - \\
\hline Case 2 & - & - & $<0.001$ \\
\hline Case 3 & 0.23 & - & - \\
\hline \multicolumn{4}{|c|}{$\begin{array}{l}\text { Proportion of those who would recommend tracheostomy at } 3 \\
\text { weeks, by sub-specialty }\end{array}$} \\
\hline Pediatric Intensive Care & $19 / 43(44.2)$ & $12 / 61(19.7)$ & $24 / 59(40.7)$ \\
\hline Neonatology & $9 / 39(23.1)$ & $1 / 1(100.0)_{m=1}^{m}$ & $1 / 2(50.0)_{m=1}$ \\
\hline Respirology & $10 / 26(38.5)_{m=1}$ & $7 / 21(33.3)_{m=1}$ & $7 / 16(43.8)_{m=1}$ \\
\hline
\end{tabular}

Figure 1 - Would recommend vs. never recommend tracheostomy under various clinical circumstances when compared to baseline. Odds ratios are shown as log odds. OR > 1 signiXies an increased likelihood of recommending tracheostomy compared to single system lung disease alone

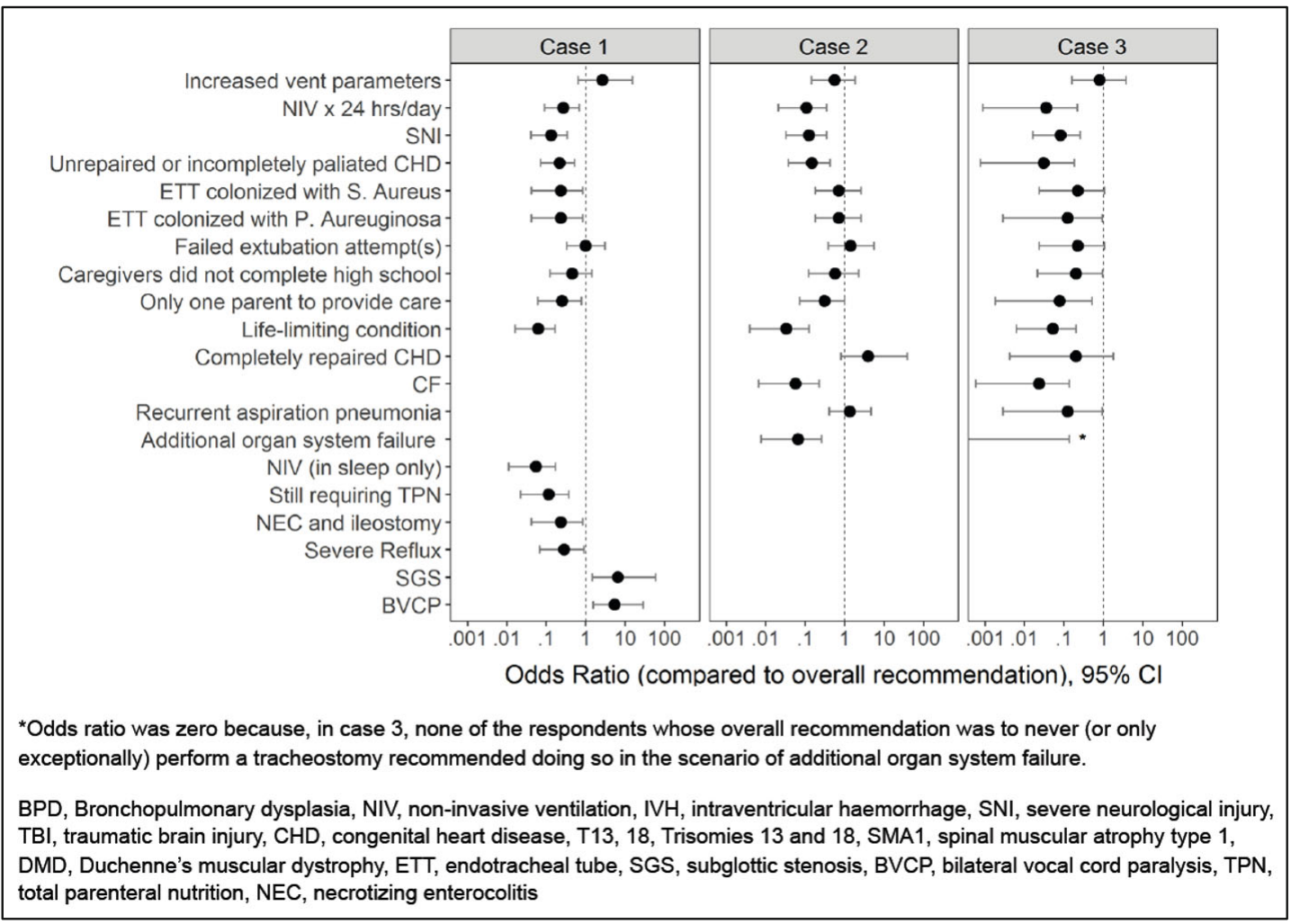


Figure 2 - Would recommend tracheostomy at 3 weeks or less, vs. longer than 3 weeks compared to baseline. Odds ratios are shown as log odds. OR $>1$ signiXies an increased likelihood of recommending earlier tracheostomy

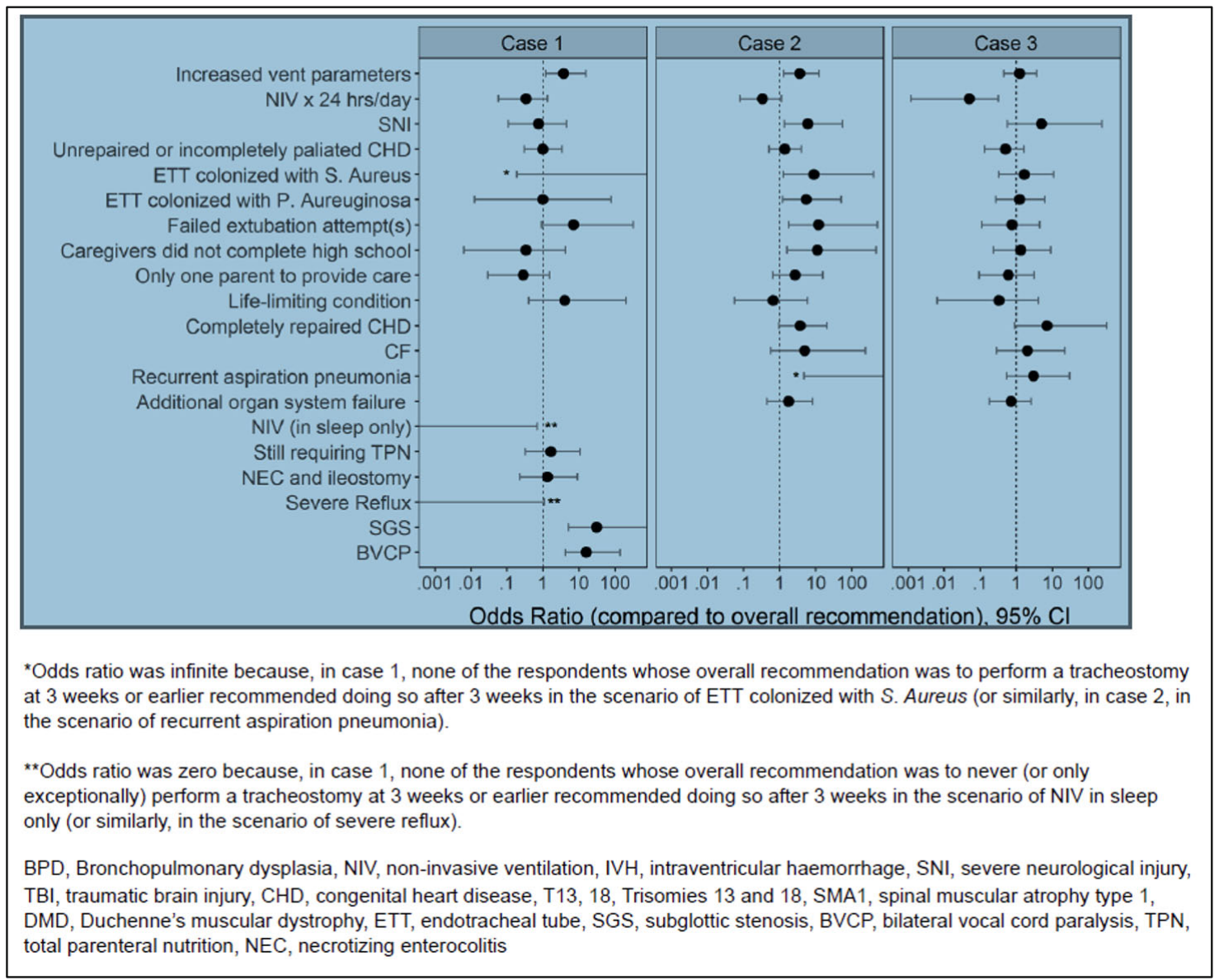

\section{References}

1. Sauthier M, Rose L, Jouvet P. Pediatric Prolonged Mechanical Ventilation: Considerations for Definitional Criteria. Respir Care. 2016;61(12):1-17. http://rc.rcjournal.com/content/early/2016/11/22/respcare.04881.abstract.

2. Holloway AJ, Spaeder MC, Basu S. Association of Timing of Tracheostomy on Clinical Outcomes in PICU Patients. Pediatr Crit Care Med. 2015;16(3):1-7. doi:10.1097/PCC.00000000000003363.

3. Fontela PS, Quach C, Karim ME, et al. Determinants of Antibiotic Tailoring in Pediatric Intensive Care: A National Survey*. Pediatr Crit Care Med. 2017;18(9):e395-e405. doi:10.1097/PCC.00000000000012384.

4. Santschi M, Randolph AG, Rimensberger PC, Jouvet P. Mechanical Ventilation Strategies in Children With Acute Lung Injury. Pediatr Crit Care Med. 2013;14(7):e332-e337. doi:10.1097/PCC.0b013e31828a89a2 
Humanization of Critical Care - Psychological Effects on Healthcare Professionals \& Relatives: A Systematic Review

Leitch, Jordan ${ }^{1,2}$; Gill, Rebecca3; Poser, Katherine ${ }^{4}$; McKeown, Sandra²; Galvin, Imelda ${ }^{1,2}$

1 Department of Anesthesiology \& Perioperative Medicine and Department of Critical Care Medicine, Kingston Health Sciences Centre, Kingston, Ontario, Canada

2 Queens University, Kingston, Ontario, Canada

3 Critical Care, Kidd 2 ICU, Kingston Health Sciences Centre, Kingston, Ontario, Canada

4 St Lawrence College, Kingston, Ontario, Canada

Introduction: Dehumanization is the process of depriving someone of positive human qualities. It is usually seen as an overt, active, extreme, conflict driven phenomenon, ${ }^{1}$ and not typically thought of as being associated with health care. There is however a more subtle form of dehumanization called infrahumanization that can occur outside conflict situations. Infrahumanization can occur whenever groups identify others as being outside their cohort and has been shown to occur in medical settings. ${ }^{2,3}$ Capozza found that physicians and nurses caring for oncology patients perceived their own professional group as possessing more uniquely human traits than the patient group. ${ }^{2}$ A study of a variety of nursing showed that denial of uniquely human traits (reasoning, rationality, morality and intellectual ability) to patients was associated with lower levels of stress among nurses with high levels of organizational and affective commitment, suggesting that infrahumanization is a subconscious coping strategy to reduce the emotional burden of caring for patients. ${ }^{3}$ Those suffering from critical illness are particularly vulnerable to this unintentional dehumanization. In an effort to refocus care of the critically ill to a more person-centered lens, several initiatives have been advocated (Figure 1), including diaries, liberal visitation policies, involvement of relatives in basic patient care and opportunities for relatives to be present during resuscitation. ${ }^{4,5,6}$ There seems to be a conflict between what feels intuitively right (to humanize patients) and what health care professionals do to protect themselves against burnout and emotional burden (dehumanize patients). Equally important is what effect these interventions may have on family members.

Objectives: To systematically review and evaluate the effects of humanized care of the critically ill, on empathy among health care professionals, anxiety among relatives and burnout and compassion fatigue in both groups.

Methods: MEDLINE, PsycINFO, Embase, CINAHL, Cochrane Central Register of Controlled Trials (CENTRAL), ProQuest Dissertations were searched from inception to 29th June 2017 for studies that investigated the effects of interventions with potential to humanize care of the critically ill, on the following outcomes: empathy among critical care professionals, anxiety among relatives and burnout and compassion fatigue in either group. We defined a humanizing intervention as: one with substantial potential to increase physical or emotional proximity to the patient. Two reviewers independently selected studies, extracted data, assessed risk of bias and data quality.

Results: Twelve studies addressing four discrete interventions (liberal visitation, diaries, family participation in basic care and witnessed resuscitation) and one mixed intervention were included. Ten studies measured anxiety among 1055 relatives. Two studies measured burnout in $\mathbf{2 8 8}$ critical care professionals. None addressed empathy or compassion fatigue. Eleven of the included studies had an overall high risk of bias. No pooled estimates of effect were calculated, as a priori criteria for data synthesis were not met.

Conclusions: We found insufficient evidence to make any quantitative assessment of the effect of humanizing interventions on any of these psychological outcomes. We observed a trend towards reduced anxiety among family members who participated in 
basic patient care, liberal visitation and diary keeping. We found conflicting effects of liberal visitation on burnout among health care professionals.

Figure 1 - Humanizing Interventions

\section{Health Care Professional Engagement}

Humanized briefing on rounds, Encouragement to use patients name, Involvement in diary keeping

Environmental

Photographs, Posters providing background information about who the person is, Family photos, Drawings, Personal items, Pet visits, Music

Revitalization

Sedation minimized, Mobilization optimized, Hair dressing, Own clothes

Patient \&Family Engagement

Open visitation policy, Family presence on rounds or during resuscitation, Diaries,

Family involvement in care

\section{References}

1. Haslam N, Loughnan S. Dehumanization and infrahumanization. Annu Rev Psychol 2014; 65:399-423.

2. Capozza D, Visintin EP, Falvo R, Testoni I. Deumanizzazione del paziente oncologico nei contesti medici. Salute e società 2015;13: 74-86.

3. Trifiletti E, Di Bernardo GA, Falvo R, Capozza D. Patients are not fully human: a nurse's coping response to stress. Journal of Applied Social Psychology 2014 1;44 (12):768-77.

4. Le Calle GH, Oviés AA, Gómez Tello V. A plan for improving the humanisation of intensive care units. Intensive Care Med 2017; 43:547-9.

5. Bäckman CG, Walther SM. Use of a personal diary written on the ICU during critical illness. Intensive Care Med. 2001; 27:426-9.

6. Oczkowski SJ, Mazzetti I, Cupido C, Fox-Robichaud AE. Family presence during resuscitation: A Canadian Critical Care Society position paper. Can Respir J 2015; 22:201-5. 
Identification of mechanistic pathways that lead to Disseminated Intravascular Coagulation (DIC) in Sepsis: Implications for screening

Chorneni, Nicholas J. ${ }^{1,2}$; Dwivedi, Dhruva J.2; Kwong, Andrew C. ${ }^{2}$; Zamir, Nasim²; FoxRobichaud, Alison E. ${ }^{1,2}$; Liaw, Patricia C. ${ }^{1,2}$

1 Department of Medicine, McMaster University, Hamilton, Ontario, Canada

2 Thrombosis and Atherosclerosis Research Institute, Hamilton, Ontario, Canada

Introduction: Disseminated intravascular coagulation (DIC) is an acquired syndrome characterized by widespread intravascular activation of coagulation complicating many conditions including sepsis, trauma, and malignancy. The International Society on Thrombosis and Haemostasis (ISTH) scoring system for diagnosis of DIC is based on the platelet count, prothrombin time, fibrinogen levels, and fibrin-related markers, with a score $\geq 5$ indicating DIC. However, once identified, the condition may already be irreversible and thus may not be responsive to therapy. To date, no useful biomarkers have been identified to differentiate "non-DIC" (a transient hypercoagulable state that does not lead to DIC) from "pre-DIC" (a hypercoagulable state which will lead to DIC). In sepsis, cell-free DNA (cfDNA) released from activated neutrophils have been shown to play an important role in the pathogenesis of DIC via pro-coagulant and antifibrinolytic effects which may contribute to microvascular thrombosis. The hypercoagulable and anti-fibrinolytic state in sepsis-associated DIC is accompanied by the increased consumption of anticoagulants such as protein $C$ (PC). High lactate concentrations are indicative of tissue hypoperfusion and are also associated with poor outcome in sepsis.

Objectives: To identify mechanistic pathways that lead to DIC in sepsis. Specifically, we will determine if abnormalities in cfDNA, PC, and/or lactate can be used to differentiate pre-DIC from non-DIC.

Methods: We studied a cohort of 173 septic patients recruited from hospitals in Hamilton, Ontario as part of the DYNAMICS Study (DNA as Prognostic Marker in ICU patients' study, Clinical Trials.gov Identifier: NCT01355042). Patient plasma samples were collected daily for the first week and then weekly thereafter. The development of DIC was scored using the ISTH diagnostic criteria on Day 1 and day a plasma sample was available. Potential markers of DIC development were either extracted from existing clinical data or measured using in-house laboratory assays.

Results: 62 of the 173 septic patients were classified as having DIC ( $n=42$ on Day 1 and $n=20$ after Day 1). Multivariable analysis demonstrated that both the presence of DIC (HR=1.98; 95\% $\mathrm{Cl}=1.04-3.79, \mathrm{p}=0.039)$ and a history of chronic lung disease $(\mathrm{HR}=1.97 ; 95 \% \mathrm{Cl}=1.02-3.80 ; \mathrm{p}=0.044)$ significantly increased mortality. Patients with DIC had higher baseline APACHE II scores (28.38 vs. 24.2, $p=0.016)$. Creatinine levels on Day $1(p<0.001)$ and Day $4(p<0.01)$ were significantly higher in patents with DIC, while lactate on Day 1 was significantly higher in patients with DIC $(p<0.001)$. Levels of PC were significantly lower in patients with DIC on all days during the first week of admission $(p<0.01)$, while cfDNA did not significantly differ between DIC and non-DIC patients at any time point. When considering those who developed DIC later, the area under the curve (AUC) for Receiver Operating Characteristic (ROC) Curves for the prediction of DIC are summarized in Table 1. The combination of PC, lactate, and platelet count yielded stronger predictive power $(\mathrm{AUC}=0.72 ; 95 \% \mathrm{Cl}, 0.59-0.85$ ) than individual markers.

Conclusion: DIC represents a significant cause of mortality in sepsis, and its pathophysiology reflects consumptive coagulopathy as indicated by reduced PC. Screening for DIC in septic patients using lactate, $\mathrm{PC}$, and platelets may prove useful in identifying patients at risk of developing DIC. 
Table 1 - Markers for predicting the development of DIC after Day 1

\begin{tabular}{|l|l|l|}
\hline Marker & Area Under Curve & $95 \% \mathrm{Cl}$ \\
\hline $\begin{array}{l}\text { The combination of PC, } \\
\text { lactate, and platelet count }\end{array}$ & 0.72 & $0.59-0.85$ \\
\hline Protein C & 0.69 & $0.57-0.80$ \\
\hline Lactate & 0.64 & $0.51-0.78$ \\
\hline Platelet count & 0.67 & $0.54-0.80$ \\
\hline cfDNA & 0.55 & $0.40-0.70$
\end{tabular}


Incorporating Laboratory Data into Machine Learning Models Improves Predictions of In-Hospital Morality After Rapid Response Team Call: A Follow-up Study

Reardon, Peter M. ${ }^{1,2}$; Parimbelli, Enea ${ }^{3}$; Michaelowski, Wojtek ${ }^{3}$; Murphy, Kyle ${ }^{1}$; Shen, Jennifer ${ }^{1}$; Herrit, Brent ${ }^{1}$; Gershkovich, Benjamin ${ }^{1}$; Wilk, Szymon ${ }^{3}$; Tanuseputro, Peter ${ }^{4}$; Kyeremanteng, Kwadwo ${ }^{1,4}$

1 Division of Critical Care, Department of Medicine, University of Ottawa, Ottawa, Ontario, Canada

2 Department of Emergency Medicine, University of Ottawa, Ottawa, Ontario, Canada

3 Telfer School of Management, University of Ottawa, Ottawa, Ontario, Canada

4 Division of Palliative Care, Department of Medicine, University of Ottawa, Ottawa, Ontario, Canada

5 Ottawa Hospital Research Institute, Ottawa, Ontario, Canada

6 Institute for Clinical and Evaluative Sciences, Ottawa, Ontario, Canada

Introduction: Clinical decision-making is challenging in the rapid response team (RRT) setting due to limited decision time and rapidly progressing illness. ${ }^{1}$ Machine learning models (MLM) are relatively new in acute care, but have shown promise as clinical decision aids. ${ }^{2,3}$ A recent novel MLM was developed to examine predictors of mortality among patients seen by the RRT. ${ }^{4}$ This model outperformed the National Early Warning Score with an AUC of 0.78.4 However, it is hypothesized that laboratory investigations and clinical history may improve the accuracy of the MLM.

Objectives: We developed a MLM for in-hospital mortality prediction among patients seen by the RRT with the goal of assessing the effect of augmenting the model by adding laboratory results and clinical history.

Methods: We performed a retrospective analysis on patients seen by the RRT between May 2012 and May 2016 at two tertiary care hospitals. We gathered information on patients using the Ottawa Hospital Data Warehouse, and supplemented with an additional chart review to obtain details regarding the RRT activation and the patient's clinical history. We derived and internally validated a machine learning model using a Gradient Boosted Decision Tree model to predict a primary outcome of 30-day in-hospital mortality. We then augmented the model with laboratory investigations and clinical history to our model. We also used a subset of patients to compare our model's accuracy to the one recently developed by Shappell et al. ${ }^{4}$

Results: A total of 6131 RRT activations occurred during the time period. A highquality subset of 256 patients with complete data including investigations and clinical history was selected for model development. The median age of 68 (IQR $57-79$ ) and median days since admission of 3 (IQR $1-8$ ). The 30 -day mortality rate was $27.7 \%$. Our base model performed with an AUC of $0.72(95 \% \mathrm{Cl} 0.57-0.87)$. When the model was augmented with laboratory investigations, clinical history, or both, the AUC improved to 0.78 (95\% Cl $0.64-0.91), 0.73$ (95\% Cl $0.58-0.88)$, and $0.77(95 \% \mathrm{Cl}$ $0.63-0.91)$ respectively. Important base mortality predictors in the model were the temperature, length of service prior to RRT call, systolic blood pressure, and age. Important laboratory markers in the augmented model were anion gap, lactate, and bicarbonate. The highest weighted clinical history variable was advanced malignancy. A sample of 50 patients were run through the Shappell et al. model, which performed with an AUC of $0.72(95 \% \mathrm{Cl} 0.57-0.87)$ in our cohort, which is similar to our base model.

Conclusion: Machine learning models can identify mortality predictors among RRT patients and potentially supplement clinical decision making. Models that incorporate laboratory investigations and clinical history may improve model accuracy. 


\section{References}

1. Giannini A, Consonni D. Physicians' perceptions and attitudes regarding inappropriate admissions and resource allocation in the intensive care setting. $\mathrm{Br} \mathrm{J}$ Anaesth. 2006;96(1):57-62. doi:10.1093/bja/aei276.

2. Koyner JL, Carey KA, Edelson DP, Churpek MM. The Development of a Machine Learning Inpatient Acute Kidney Injury Prediction Model. Crit Care Med. 2018. doi:10.1097/CCM.0000000000003123.

3. Nemati S, Holder A, Razmi F, Stanley MD, Clifford GD, Buchman TG. An Interpretable Machine Learning Model for Accurate Prediction of Sepsis in the ICU. Crit Care Med. 2017. doi:10.1097/CCM.0000000000002936.

4. Shappell C, Snyder A, Edelson DP, Churpek MM. Predictors of In-Hospital Mortality After Rapid Response Team Calls in a 274 Hospital Nationwide Sample. Crit Care Med. 2018:1. doi:10.1097/CCM.0000000000002926. 
Inter-Rater Reliability and Responsiveness of Key Physical Functional Outcome Measures in ICU Survivors

Costigan, $\mathrm{FA}^{1}$; Rochwerg, $\mathrm{B}^{2}$; Molloy, $\mathrm{AJ}^{1}$; McCaughan, $\mathrm{M}^{1}$; Farley, $\mathrm{C}^{3}$; Millen, $\mathrm{T}^{3}$; Reid, $\mathrm{JC}^{4} ; \mathrm{Kh}, \mathrm{ME}^{4}$

1 Department of Physiotherapy, St. Joseph's Healthcare Hamilton, Ontario, Canada

2 Division of Critical Care, Department of Medicine, McMaster University, Hamilton, Ontario, Canada

3 Juravinski Hospital, Hamilton, Ontario, Canada

4 School of Rehabilitation Sciences, McMaster University, Hamilton, Ontario, Canada

Introduction/Background: With more patients surviving ICU due to advances in critical care, an increased number are also at risk for physical function impairments that can last for up to 8 years after ICU discharge. Effective interventions to maintain or improve physical function post-ICU are urgently needed. In parallel, psychometrically rigorous measures to assess the effectiveness of these interventions are required. There is no consensus on the optimal instrument(s) to assess physical function in ICU survivors. Simple one-item measures of physical tasks typically incorporated into therapy sessions may be the most attractive candidates given their ease of use and limited need for training of assessors. It is imperative that we ensure that potential measures are adequately tested to document reliability and responsiveness.

Objectives: To train front-line clinicians to administer and to prospectively analyze the inter-rater reliability of 3 physical function measures in ICU survivors: Physical Function ICU Test-scored (PFIT-s), the 30 second sit-to-stand (30STS), and the two-minute walk test (2MWT).

Methods: We conducted a two-centre, prospective observational study with adult medical-surgical ICU patients in Hamilton, Ontario. Inclusion criteria: Patients with an ICU length of stay of 3 days or greater, who were mechanically ventilated for at least 24 hours, and who were able to ambulate independently with or without a gait aid prior to hospital admission. Outcome measures were the 4-item PFIT-s (10 points), the 30STS (\# of stands), and the 2MWT (distance in $\mathrm{m}$ ). For each patient, 2 blinded assessments were collected weekly within 24 hours of each other from ICU discharge to hospital discharge. We engaged multiple raters at each site; clinical schedules determined the rater for each assessment. We calculated inter-rater reliability using the intra-class correlation coefficient (ICC) for the total score for each measure using a 2way random effects model. We also calculated the standard error of the measurement (SEM), and the minimal detectable change at 90\% confidence (MDC 90 ). For continuous variables, we calculated the mean and standard deviation (SD) or median and interquartile range (IQR) if not normally distributed.

Results: Each of 20 therapists across 2 sites participated in a 1.5 hour long training session. We enrolled 42 patients, and 40 had at least 1 measure collected. Our consent rate was $89 \%$. Enrolled patients had a mean (SD) age of $62(17)$ years, $53 \%$ were female, $73 \%$ medical admissions, and had a median (IQR) APACHE II score of 20 (14). Median (IQR) ICU and hospital length of stay were 7 (8) and 22 (32) days respectively. For the PFIT-s, 36 patients had 66 paired scores; ICC was $0.78(0.66$, 0.86 ), SEM was 1.04 , and MDC 90 was 2.42. For the 30 STS, 35 patients had 67 paired scores; ICC was $0.85(0.76,0.90)$, SEM was 1.91 , and MDC 90 was 4.45 . For the 2 MWT, 35 patients had 58 paired scores; ICC was $0.78(0.66,0.87)$, SEM was 20.87 , and MDC 90 was 48.69.

Conclusions: The PFIT-s, the 30STS, and the 2MWT, measured weekly across the hospital stay in ICU survivors, show good inter-rater reliability and require little additional assessor training as they consist of tasks that fit naturally into clinical practice. This study is an important step in addressing the need for a reliable, valid and practical core set of outcome measurements to monitor the continuum of patient recovery following an ICU stay. 
Interventions to Improve Surrogate Decision-Making in Intensive Care Units: A Systematic Review and Meta-Analysis of Randomized Controlled Trials

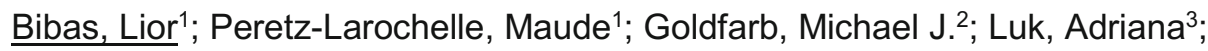
Englesakis, Marina ${ }^{4}$, Michael E. Detsky ${ }^{1,5}$, Neill K. Adhikari ${ }^{1,5}$; Patrick R. Lawler ${ }^{3}$ 1 Interdepartmental Division of Critical Care, University of Toronto, Toronto, Ontario, Canada

2 Division of Cardiology, Department of Medicine, McGill University, Montreal, Quebec, Canada.

3 Peter Munk Cardiac Centre and Division of Cardiology, Toronto General Hospital/University Health Network, Toronto, Ontario, Canada

4 Library and Information Services, University Health Network, Toronto, Ontario, Canada

5 Department of Critical Care Medicine and Sunnybrook Research Institute, Sunnybrook Health Sciences Centre, Toronto, Ontario, Canada

6 Sinai Health System, Toronto, Ontario, Canada

Introduction: It is uncertain whether targeted interventions to improve surrogate decision-making in intensive care units (ICUs) reduce non-beneficial treatment and improve surrogate decision-maker (SDM) comprehension, satisfaction, and related outcomes. Several randomized clinical trials (RCTs) have observed inconsistent results.

Objective: We performed a systematic review and meta-analysis to assess the efficacy of such interventions and to identify key components of successful interventions.

Methods: We searched OVID versions of MEDLINE, EMBASE, Ovid Nursing Database, Cochrane Central Register of Controlled Trials, and Cochrane Database of Systematic Reviews, and PubMed, for potentially relevant studies. We included RCTs that included outcomes of interest in three categories: (1) patient centered (mortality, length of stay [LOS], use of life-sustaining therapies [mechanical ventilation, vasopressors, nutritional support]); (2) SDM -centered (comprehension, change in decision on goals of care, development of psychological comorbidities, satisfaction with care); and (3) resource utilization (cost of care, health care professional use). Data were pooled using random effects meta-analysis to account for potential inter-study heterogeneity (RevMan). Binary outcomes were summarized as risk ratios (RR) and continuous outcomes as mean differences (MD), with 95\% confidence intervals (Cl). Heterogeneity was assessed using the 12 measure. Data from cluster RCTs was adjusted for design effect using their respective Intracluster Correlation Coefficient. Results: After screening 3735 citations, 13 RCTs met inclusion criteria. Interventions were categorized as: healthcare professional-led $(n=6)$, ethics consultation $(n=3)$, palliative care consultation $(n=2)$ and media $(n=2)$. Meta-analysis revealed no difference in mortality (RR 1.03; 95\% Cl 0.98-1.08; I2=0\%; 10 RCTs) or ICU LOS among all patients (MD -0.79 days; $95 \% \mathrm{Cl}-2.33$ to 0.76 ; I2=0; 6 RCTs). There was however a significant reduction in ICU LOS among non-survivors (MD -2.11 days; 95\% $\mathrm{Cl}-4.16$ to $-0.07 ; \mathrm{I} 2=32 \%$; $8 \mathrm{RCTs}$ ). There was no difference in satisfaction with care and conflicting results regarding psychological comorbidities (i.e. anxiety, depression and post-traumatic stress disorder). Only two trials showed improvements in comprehension with the intervention. Five trials reported on resource utilization, and only one nurse-led intervention showed a significant reduction in costs. Given the observed diversity of interventions and outcomes, it was not possible to determine key components of SDM interventions more likely to improve outcomes.

Conclusion: Diverse interventions to improve surrogate decision-making in ICU reduced ICU in deceased patients only. They did not affect mortality or ICU LOS in all patients. They also did not consistently reduce psychological comorbidities in family members or improve satisfaction with care. Future studies are needed to better 
understand the complex processes related to surrogate decision-making to improve both patient-centered and family-centered outcomes.

\section{Table 1}

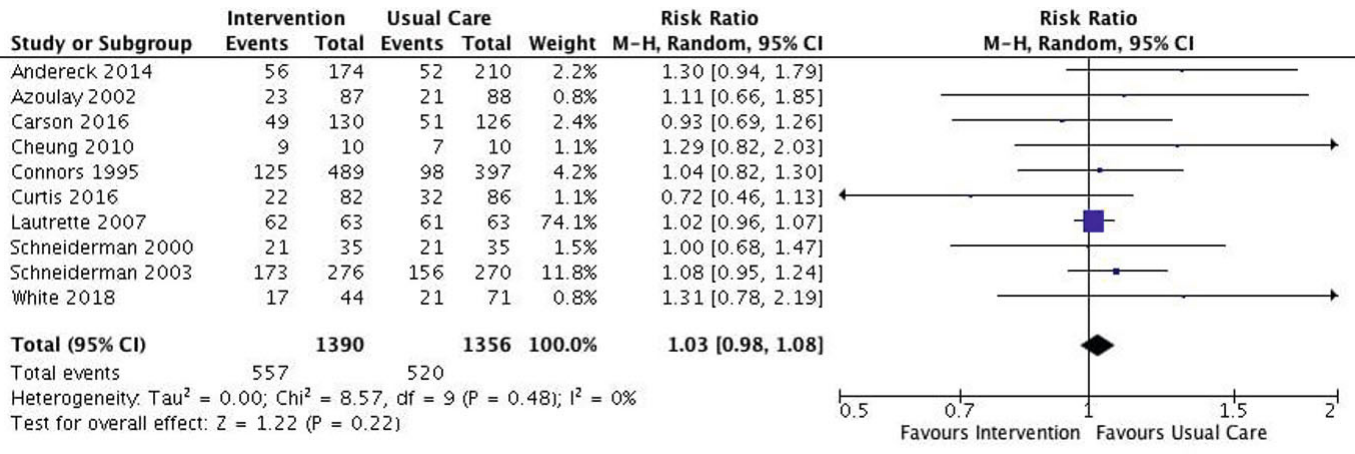

Table 2

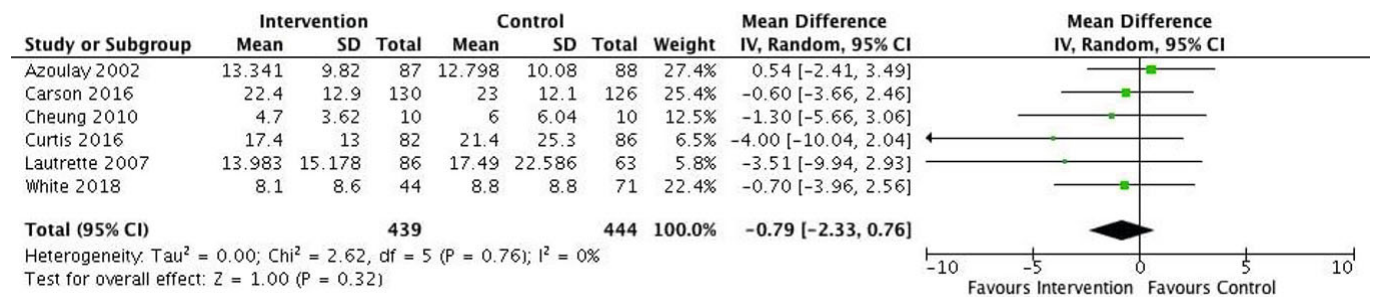

Table 3

\begin{tabular}{|c|c|c|c|c|c|c|c|c|c|c|c|}
\hline \multirow[b]{2}{*}{ Study or Subgroup } & \multicolumn{3}{|c|}{ Intervention } & \multicolumn{3}{|c|}{ Control } & \multicolumn{2}{|r|}{ Mean Difference } & \multirow{2}{*}{\multicolumn{3}{|c|}{$\begin{array}{l}\text { Mean Difference } \\
\text { IV, Random, } 95 \% \mathrm{CI}\end{array}$}} \\
\hline & Mean & SD & Total & Mean & SD & Total & Weight & IV, Random, $95 \% \mathrm{CI}$ & & & \\
\hline Andereck 2014 & 11 & 0 & 56 & 11 & 0 & 52 & & Not estimable & & & \\
\hline Azoulay 2002 & 11.864 & 6.756 & 23 & 12.667 & 8.315 & 21 & $15.1 \%$ & $-0.80[-5.31,3.70]$ & & & \\
\hline Carson 2016 & 11.2 & 8.2 & 49 & 10.9 & 13.9 & 51 & $15.3 \%$ & $0.30[-4.15,4.75]$ & & & \\
\hline Cheung 2010 & 4.4 & 3.78 & 5 & 4.83 & 6.88 & 6 & $8.6 \%$ & $-0.43[-6.86,6.00]$ & & & \\
\hline Curtis 2016 & 9.6 & 6.6 & 21 & 27 & 40 & 25 & $1.6 \%$ & $-17.40[-33.33,-1.47]$ & & & \\
\hline Lautrette 2007 & 13.66 & 16.363 & 62 & 18.378 & 24.803 & 61 & $6.7 \%$ & $-4.72[-12.16,2.72]$ & & & \\
\hline Schneiderman 2000 & 4.2 & 5.56 & 21 & 13.2 & 17.47 & 21 & $6.1 \%$ & $-9.00[-16.84,-1.16]$ & - & & \\
\hline Schneiderman 2003 & 6.42 & 6.89 & 173 & 7.86 & 10.48 & 156 & $37.2 \%$ & $-1.44[-3.38,0.50]$ & $\longrightarrow$ & & \\
\hline White 2018 & 5.5 & 5.03 & 12 & 8.93 & 10.21 & 14 & $9.5 \%$ & $-3.43[-9.49,2.63]$ & & & \\
\hline Total $(95 \% \mathrm{Cl})$ & & & 366 & & & 355 & $100.0 \%$ & $-2.11[-4.16,-0.07]$ & & & \\
\hline $\begin{array}{l}\text { Heterogeneity. Tau }{ }^{2}= \\
\text { Test for overall effect: }\end{array}$ & $\begin{array}{l}\text {.96; Chi } \\
=2.02\end{array}$ & $\begin{array}{l}2=9.14 \\
(P=0.0\end{array}$ & 4) $d f=7$ & & $24) ;\left.\right|^{2}=2$ & & & & $\begin{array}{ll}-10 & -5 \\
& -5 \\
& \text { Favours Intervention }\end{array}$ & f Favours & $\begin{array}{l}1 \\
5 \\
\text { Control }\end{array}$ \\
\hline
\end{tabular}


Introducing a Compassion-Centered End of Life Project in a Large Community ICU: Facilitators and Barriers

Levesque, $\mathrm{K}^{1}$; Dragomir, $\mathrm{D}^{1}$; Davidson $\mathrm{F}^{1}$; Richter, $\mathrm{B}^{1}$; Duan, $\mathrm{E}^{1}$; Tsang, JLY1; D'sa, $\mathrm{R}^{1}$; Young, $\mathrm{E}^{1} ;$ Cook, $\mathrm{D}^{2}$; Tam $\mathrm{B}^{1}$

1 Division of Critical Care, St. Catharines Site, Niagara Health, St. Catharines, Ontario, Canada

2 Division of Critical Care, St. Joseph's Healthcare Hamilton, Hamilton, Ontario, Canada

Introduction: Death in hospital is common but remains a difficult experience for patients, families, and clinicians. ${ }^{1,2}$ Community ICUs, like other ICUs, may deliver suboptimal end of life (EOL) care. ${ }^{3}$ Patients and families desire compassion, defined as an action-oriented expression of empathy. ${ }^{4}$ The 3 Wishes Project is a compassioncentered EOL project that aims to perform at least three acts of compassion for dying patients and/or their families. ${ }^{5}$ This project was shown to dignify the patient, create positive memories for families, and call forth compassion in clinicians. ${ }^{5}$

Objectives: The objective of this study is to understand facilitators of and barriers to implementing the 3 Wishes Project in a single center community ICU.

Methods: We piloted a compassion centered EOL project from January 1st to June 30th, 2018, and assessed its implementation with mixed methods. At inception, an environmental scan was performed with key stakeholders. Based on the perceived gaps, two interventions were applied: engagement (small group sessions, quarterly reports, 1:1 mentoring) and empowerment (resources and financial support). The main quantitative measure was monthly enrolment rate. Secondary measures included the number of staff who enrolled patients for the first time, the number of compassionate acts per patient enrolled, and the financial cost of each act of compassion. To understand experience during implementation, seven semi-structured interviews with key stakeholders were conducted with six clinicians who implemented the 3 Wishes Project for their patient and one clinician who was involved in the initial environmental scan. Quantitative data were analyzed using appropriate statistical process control while qualitative data were analyzed in triplicate using a qualitative descriptive approach.

Results: By the end of the pilot phase, 14 patients were enrolled and 61 wishes were implemented. $13.7 \%$ of dying patients were enrolled monthly. In addition, 9 clinicians enrolled a patient for the first time into the 3 Wishes Project. 1.5 clinicians per month enrolled a patient for the first time and 4.6 acts of compassion were performed per patient without additional financial cost. Interviews identified four facilitators: a shared vision for sparking change, research team integration with frontline staff, a collaborative learning environment, and available resources for staff to implement acts of compassion. Two barriers identified were: the differential manifestation of the project within different sub-cultures of the ICU, and the unclear process to fulfill an act of compassion. In addition, qualitative interviews suggested the possible benefits of two next steps: a point of care collaborator across nursing lines to spread awareness and expertise, and maintaining focus on the personalization of acts of compassion.

Conclusion: The pilot phase of a compassion-centered EOL project was successfully implemented for a small proportion of dying patients and their families in our community ICU. Key facilitators included a shared vision, research-clinical team partnership, a collaborative learning environment, and resources to empower staff. Key areas to be aware of during future implementation include differential uptake of interventions within sub-cultures, and maintaining a clear operational process. Based on staff feedback, we have developed the next steps to expand the 3 Wishes Project in our community ICU. 


\section{References}

1. Reyniers T, Houttekier D, Cohen J, Pasman HR, Deliens L. The acute hospital setting as a place of death and final care. A qualitative study on perspectives of family physicians, nurses and family carers. Health \& Place. 2014;27(C):77-83. doi:10.1016/j.healthplace.2014.02.002.

2. Heyland DK, Lavery JV, Tranmer JE, Shortt SE, Taylor SJ. Dying in Canada: is it an institutionalized, technologically supported experience? J Palliat Care. 2000;16 Suppl:S10-S16.

3. Sarti AJ, Bourbonnais FF, Landriault A, Sutherland S, Cardinal P. An Interhospital, Interdisciplinary Needs Assessment of Palliative Care in a Community Critical Care Context. J Palliat Care. 2015;31(4):234-242. doi:10.1177/082585971503100405.

4. Sinclair S, Beamer K, Hack TF, et al. Sympathy, empathy, and compassion: A grounded theory study of palliative care patients' understandings, experiences, and preferences. Palliative Medicine. 2017;31(5):437-447. doi:10.1177/0269216316663499.

5. Cook D, Swinton M, Toledo F, et al. Personalizing Death in the Intensive Care Unit: The 3 Wishes Project. Ann Intern Med. 2015;163(4):271-10. doi:10.7326/M15-0502. 
Medical Fragility and Inpatient Mortality in a Quaternary Care Pediatric ICU

Nicoll, J' ${ }^{1,3,4} ;$ Frndova, $\mathrm{H}^{1}$; Dryden-Palmer, $\mathrm{K}^{1,3}$; Moga, M-A ${ }^{1,2}$; Parshuram, $\mathrm{C}^{1,3,4}$

1 Department of Critical Care Medicine, Hospital for Sick Children, Toronto, Ontario, Canada

2 Heart Centre, Division of Cardiology in the Department of Pediatrics at the Hospital for Sick Children, Toronto, Ontario, Canada

3 Centre for Safety Research, Research Institute, The Hospital for Sick Children, Toronto, Ontario, Canada

4 Institute of Health Policy, Management and Evaluation, University of Toronto, Toronto, Ontario, Canada

Introduction: In developed countries, PICU survival rates exceed $97 \%$ with increased survivor disability, hospital readmissions and PICU admissions. ${ }^{1-4}$ Up to two thirds of PICU patients may have a chronic medical condition. ${ }^{5,6} \mathrm{~A}$ subset among these can be characterized as having medical fragility, which encompasses technology dependence, severe neurodevelopmental impairment or three or more chronic medical conditions. ${ }^{7,8}$ However, the prevalence of medically fragile in PICU and their prognosis after critical illness remains poorly understood.

Objectives: Our objective was to describe the prevalence of device dependent patients and their inpatient mortality during a 14-year period in a quaternary Critical Care Unit

Methods: We conducted a retrospective cohort study using the electronic medical records for all patients born after January 1st, 2001 admitted to the Critical Care Unit (CCU) at the Hospital for Sick Children between 2001 to 2015. We used SAS software (SAS Institute, Toronto, Ontario) to assess the median number of patients per year and overall with each device, inpatient mortality, CCU admissions, CCU and hospital length of stay, ICU patient days and number of admissions per patient.

Results: There were 11973 patients admitted 17889 times for 110573 ICU days. With time, the number of ICU admissions increased and length of stay decreased. The median (IQR) length of stay was 2.8 (1.0-7.9) days. Overall, at time of ICU discharge 315 patients $(2.6 \%)$ had noninvasive ventilation, $136(1.1 \%)$ had a tracheostomy, 61 $(0.5 \%)$ had a tracheostomy and were ventilated and $15(0.1 \%)$ were dialyzed. The prevalence of tracheostomy increased with time, and comprised 7408 (6.7\%) patient days in the cohort. Inpatient mortality decreased over time. Inpatient mortality was $6.7 \%$ overall, $7 \%$ in patients with tracheostomy, $5.9 \%$ with non-invasive ventilation and $8.2 \%$ with a tracheostomy and ventilation.

Conclusion: Device dependent patients comprised a small proportion of ICU admissions, that is increasing with time and is associated with a disproportionate number of ICU days. Inpatient mortality was similar to the entire cohort. A greater understanding of the prognosis of this population after critical illness is important for planning the future of Critical Care.

\section{References}

1. Namachivayam $P$, Shann $F$, Shekerdemian $L$, et al. Three decades of pediatric intensive care: Who was admitted, what happened in intensive care, and what happened afterward. Pediatr Crit Care Med 2010;11:549-55.

2. Choong $\mathrm{K}, \mathrm{Al}-\mathrm{Harbi} \mathrm{S}$, Siu $\mathrm{K}$, et al. Functional recovery following critical illness in children: the "wee-cover" pilot study. Pediatr Crit Care Med 2015;16:310-8.

3. Pollack MM, Holubkov R, Funai T, et al. Pediatric intensive care outcomes: development of new morbidities during pediatric critical care. Pediatr Crit Care Med 2014;15:821-7. 
4. Typpo KV, Petersen NJ, Hallman DM, Markovitz BP, Mariscalco MM. Day 1 multiple organ dysfunction syndrome is associated with poor functional outcome and mortality in the pediatric intensive care unit. Pediatr Crit Care Med 2009;10:562-70.

5. Edwards JD, Houtrow AJ, Vasilevskis EE, et al. Chronic conditions among children admitted to U.S. pediatric intensive care units: their prevalence and impact on risk for mortality and prolonged length of stay*. Crit Care Med 2012;40:2196-203.

6. Cremer R, Leclerc F, Lacroix J, Ploin D, Group GRCDiPS. Children with chronic conditions in pediatric intensive care units located in predominantly French-speaking regions: Prevalence and implications on rehabilitation care need and utilization. Crit Care Med 2009;37:1456-62.

7. Peterson-Carmichael SL, Cheifetz IM. The chronically critically ill patient: pediatric considerations. Respir Care 2012;57:993-1002; discussion -3.

8. Cohen E, Kuo DZ, Agrawal R, et al. Children with medical complexity: an emerging population for clinical and research initiatives. Pediatrics 2011;127:529-38. 
Melatonin and Melatonin Agonists as Adjuncts for Sleep and Sedation in Critical Illness: A Systematic Review

Farina, Sasha ${ }^{1}$; Foster, Jennifer ${ }^{2,3,4}$; Singh, Tishaan ${ }^{1}$; Rose, Louise; ;,6,7,8,9; Burry, Lisa ${ }^{10}$ 1 Leslie Dan Faculty of Pharmacy, University of Toronto, Toronto, Ontario, Canada 2 Department of Paediatrics, Schulich School of Medicine \& Dentistry, Western University, London, Ontario, Canada

3 IWK Health Centre, Dalhousie University, Halifax, Canada

4 Division of Children's Health \& Therapeutics, Children's Health Research Institute, London, Canada

5 Department of Pharmacy, Mount Sinai Hospital, Toronto, Ontario, Canada

6 Department of Critical Care Medicine, Sunnybrook Health Sciences Centre, Toronto, Ontario, Canada

7 Evaluative Clinical Sciences, Trauma, Emergency \& Critical Care Research Program, Sunnybrook Research Institute, Toronto, Ontario, Canada

8 Lawrence S. Bloomberg Faculty of Nursing, University of Toronto, Toronto, Canada 9 Institute for Clinical Evaluative Sciences, Toronto, Ontario, Canada

10 Provincial Centre of Weaning Excellence, Toronto East General Hospital, Toronto, Ontario, Canada

Introduction: Critically ill patients experience poor quality of sleep and may develop abnormal sleep-related phenomena ${ }^{1,2}$. Timely management of sleep disturbances for critically ill patients is vital due to poor health outcomes such as the development of delirium, prolonged stays in the intensive care unit (ICU), and increased mortality ${ }^{3-6}$. Critically ill patients may exhibit disturbed diurnal rhythms with altered patterns of melatonin secretion. Melatonin is a molecule that exerts chronobiologic effects on sleep and biorhythms and administration of melatonin and melatonin receptor agonists (collectively referred to as MMA) have the potential to safely improve natural sleep, provide sedation, and may prevent or treat delirium ${ }^{7,8}$. We conducted a systematic review to assess the efficacy of MMAs for the promotion of sleep and reduction in sedative use in the ICU.

Methods: We performed a systematic search of the following databases: MEDLINE, EMBASE, the Cochrane database, Web of Science, CINAHL, PsycINFO, Scopus, ClinicalTrials.gov and clinical practice guidelines. No language restrictions were applied and all study designs were eligible except for case studies. Studies could include critically ill adults and or children but not neonates. Two reviewers independently screened the retrieved articles for studies that evaluated the use of melatonin for sleep or reduction of sedatives in critically ill patients. Data from eligible studies is being extracted and after the quality assessment, data was pooled for statistical analysis if at least 2 trials reported the outcome of interest.

Results: Our search strategy yielded 1431 citations, of which 1391 were excluded based on title and abstract. After full-text reviews of 40 citations, we identified 6 eligible RCTs with a total of 343 patients; 5 ongoing RCTs were identified. All included trials examined melatonin compared to either a placebo, a benzodiazepine, or non-drug sleep strategy. We found no difference in quantity of sleep between those who received melatonin and those who did not (mean difference -0.01 , moderate quality evidence ( $n=3$ studies, 119 subjects). We also found no difference in duration of mechanical ventilation or ICU length of stay. There was insufficient data to pool on sleep quality, agitation, or sedative drug consumption. Adverse events were rarely identified.

Conclusions: We found limited moderate-quality evidence that melatonin does not alter sleep quantity compared to non-melatonin strategies. Furthermore, we found the methods for assessing sleep and defining sedative consumption varied between trials limiting our ability to pool results. 


\section{References}

1. Bourne RS, Mills GH, Minelli C. Melatonin therapy to improve nocturnal sleep in critically ill patients: encouraging results from a small randomised controlled trial. Critical Care 2008;12:R52.

2. Ferracioli-Oda E, Qawasmi A, Bloch MH. Meta-analysis: melatonin for the treatment of primary sleep disorders. PloS one 2013;8:e63773.

3. Boyko Y, Ørding H, Jennum P. Sleep disturbances in critically ill patients in ICU: how much do we know? Acta anaesthesiologica scandinavica 2012;56:950-8.

4. Delaney LJ, Haren F, Lopez V. Sleeping on a problem: the impact of sleep disturbance on intensive care patients-a clinical review. Annals of intensive care 2015;5:3.

5. Ely EW, Shintani A, Truman B, et al. Delirium as a predictor of mortality in mechanically ventilated patients in the intensive care unit. Jama 2004;291:1753-62.

6. Orwelius L, Nordlund A, Nordlund P, Edéll-Gustafsson U, Sjöberg F. Prevalence of sleep disturbances and long-term reduced health-related quality of life after critical care: a prospective multicenter cohort study. Critical Care 2008;12:R97.

7. Shilo L, Dagan Y, Smorjik Y, et al. Effect of melatonin on sleep quality of COPD intensive care patients: a pilot study. Chronobiology international 2000;17:71-6.

8. Bruni O, Alonso-Alconada D, Besag F, et al. Paediatric use of melatonin (Author reply to DJ Kennaway). European journal of paediatric neurology: EJPN: official journal of the European Paediatric Neurology Society 2015;19:491. 
Melatonin and Melatonin Agonists to Prevent and Treat Delirium in Critical IIIness: A Systematic Review

$\underline{\text { Singh, Tishaan }}^{1}$; Foster, Jennifer ${ }^{2,3,4}$; Farina, Sasha ${ }^{1}$; Rose, Louise ${ }^{5,6,7,8,9} ;$ Burry, Lisa $^{1,10}$ 1 Leslie Dan Faculty of Pharmacy, University of Toronto, Toronto, Ontario, Canada 2 Department of Paediatrics, Schulich School of Medicine \& Dentistry, Western University, London, Ontario, Canada

3 IWK Health Centre, Dalhousie University, Halifax, Nova Scotia, Canada

4 Division of Children's Health \& Therapeutics, Children's Health Research Institute, London, Ontario, Canada

5 Department of Critical Care Medicine, Sunnybrook Health Sciences Centre, Toronto, Ontario, Canada

6 Evaluative Clinical Sciences, Trauma, Emergency \& Critical Care Research Program, Sunnybrook Research Institute, Toronto, Ontario, Canada

7 Lawrence S. Bloomberg Faculty of Nursing, University of Toronto, Toronto, Canada; 8 Institute for Clinical Evaluative Sciences, Toronto, Ontario, Canada 9 Provincial Centre of Weaning Excellence, Toronto East General Hospital, Toronto, Ontario, Canada

10 Department of Pharmacy, Mount Sinai Hospital, Toronto, Ontario, Canada

Introduction: Critically ill patients are at high risk of developing delirium with reported rates of up to $80 \% .^{1-3}$ Some studies have shown abnormal melatonin concentrations to be associated with post-operative and intensive care unit (ICU) delirium. ${ }^{4,5}$ Exogenous melatonin and melatonin agonists (MMA) have the potential to decrease the incidence and severity of delirium through their regulation of the circadian rhythm as well as hypnotic and sedative-sparing effects. ${ }^{4-9}$ We conducted a systematic review on the efficacy and safety of MMA for the prevention and treatment of ICU delirium.

Objectives: To assess 1) the effectiveness of MMA compared to placebo or alternative therapy for preventing and treating delirium in critically ill patients and to examine the effect of MMA administration on: 2) duration of delirium; 3) delirium-free and coma-free days; 4) duration of mechanical ventilation; 5) ICU and hospital length of stay; 6) mortality; 7) discharge disposition; 8) long term cognitive outcomes; and 9) adverse events.

Methods: We used methods established by Cochrane to conduct this review. We searched the following databases (inception-June 2018) without language restriction: MEDLINE, Embase, PsychINFO, Cochrane Library, CINAHL, Web of Science, Scopus, and grey literature. We included all randomized (RCT) and non-randomized studies of critically ill patients that assessed MMA for the prevention or treatment of ICU delirium. Two reviewers independently screened the retrieved articles. Data from eligible studies was extracted and pooled for statistical analysis if at least 2 trials reported the outcome of interest.

Results: We screened 638 records and included 6 published studies $(n=1152$ subjects); we identified 5 conference abstracts and 13 ongoing studies for future use. Of the 6 included studies, 4 were RCTs, 1 was a prospective observation study, and 1 was a retrospective chart review. Three studies evaluated ramelteon, 2 melatonin, and $1 \mathrm{~L}$-tryptophan. We found MMA reduced the incidence of delirium (RR $0.53,95 \% \mathrm{Cl}$ $0.31,0.90 ; 6$ trials; moderate quality evidence). We found no difference in the duration of delirium (MD $-0.91,95 \% \mathrm{Cl}-2.58,0.76 ; 3$ trials; moderate quality evidence). We also found no difference in the duration of mechanical ventilation, lengths of stay, or mortality. No trial reported on delirium or coma-free days, discharge disposition or cognitive outcomes. Adverse events were rarely identified.

Conclusion: We found moderate-quality evidence that MMAs prevent delirium in critically ill patients. We found moderate quality evidence that MMAs do not shorten the duration of delirium or alter duration of mechanical ventilation. 


\section{References}

1. Bellapart J, Boots R. Potential use of melatonin in sleep and delirium in the critically ill. Br J Anaesth. 2012;108(4):572-80.

2. Daoud A, Duff JP, Joffe AR, Alberta SN. Diagnostic accuracy of delirium diagnosis in pediatric intensive care: a systematic review. Crit Care. 2014;18(5):489.

3. Mistraletti G, Carloni E, Cigada M, Zambrelli E, Taverna M, Sabbatici G, et al. Sleep and delirium in the intensive care unit. Minerva Anestesiol. 2008;74(6):329-33.

4. Miyazaki T, Kuwano H, Kato H, Ando H, Kimura H, Inose $\mathrm{T}$, et al. Correlation between serum melatonin circadian rhythm and intensive care unit psychosis after thoracic esophagectomy. Surgery. 2003;133(6):662-8.

5. Yoshitaka S, Egi M, Morimatsu H, Kanazawa T, Toda Y, Morita K. Perioperative plasma melatonin concentration in postoperative critically ill patients: its association with delirium. J Crit Care. 2013;28(3):236-42.

6. Al-Aama T, Brymer C, Gutmanis I, Woolmore-Goodwin SM, Esbaugh J, Dasgupta $M$. Melatonin decreases delirium in elderly patients: a randomized, placebocontrolled trial. Int J Geriatr Psychiatry. 2011;26(7):687-94.

7. Bruni O, Alonso-Alconada D, Besag F, Biran V, Braam W, Cortese S, et al. Paediatric use of melatonin (author reply to D. J. Kennaway). Eur J Paediatr Neurol. 2015;19(4):491-3.

8. Wyatt JK, Dijk DJ, Ritz-de Cecco A, Ronda JM, Czeisler CA. Sleep-facilitating effect of exogenous melatonin in healthy young men and women is circadian-phase dependent. Sleep. 2006;29(5):609-18.

9. Uchida K, Aoki T, Ishizuka B. Postoperative delirium and plasma melatonin. Med Hypotheses. 1999;53(2):103-6. 
Mesenchymal Stromal/Stem Cells Enhance Bacterial Killing Partly Via Hemoxygenase-1 Expression in Macrophages from Healthy and Septic Patients and Accelerate Bacterial Clearance and Recovery in Preclinical Sepsis Model

Jerkic, Mirjana ${ }^{1}$; Gagnon, Stéphane ${ }^{1}$; Ormesher, Lindsay ${ }^{1}$; Goyal, Sakshi'; Rabani, Razieh $^{1}$; Kavanagh, Brian ${ }^{2,3}$; Curley, Gerard F1; Laffey, John $\mathrm{G}^{1,4}$

1 Department of Anaesthesia, Critical IIIness and Injury Research Centre, Keenan Research Centre of St. Michael's Hospital, University of Toronto, Ontario, Canada 2 Translation Medicine, The Hospital for Sick Children, Toronto, Ontario, Canada 3 Departments of Critical Care and Anesthesia, Hospital for Sick Children, University of Toronto, Ontario, Canada

4 Anaesthesia, School of Medicine and Regenerative Medicine Institute, National University of Ireland, Galway, Ireland

Introduction \& Objective: Sepsis, a syndrome of life-threatening organ dysfunction caused by dysregulated host response to infection ${ }^{1,2}$, is most commonly initiated by bacterial infection of the lung and abdominal cavity. Mesenchymal Stromal/Stem Cells (MSCs) have potent immunomodulatory properties, they demonstrated therapeutic potential in animal preclinical sepsis models $\mathbf{s}^{3,4,5}$ and are in early phase clinical trials for sepsis ${ }^{6}$. We explored the role of hemoxygenase-1(HO-1) in mediating MSC-induced enhancement of $M \phi$ function in vitro and in a clinically relevant animal model of abdominal sepsis.

Methods: Blood monocytes, isolated from healthy donors and septic patients (in first $48 \mathrm{~h}$ of sepsis) were differentiated into $\mathrm{M} \phi$ and exposed to human umbilical cord (hUC) MSCs (obtained from Tissue Regeneration Therapeutics $₫$, Toronto) in transwell plates for $48 \mathrm{~h}$. M $\phi$ phagocytosis was assessed using serum opsonized zymozan (SOZ) while their killing capabilities were tested using live E. coli. Protein expression was analyzed by Western blot. M $\phi$ were also isolated from peritoneal fluid (PLF) of septic rats challenged with cecal slurry $(1 \mathrm{~g} / \mathrm{kg}$, i.p.) $48 \mathrm{~h}$ earlier, that been treated with UC-MSCs (10 mill/kg, i.v.) or placebo $4 \mathrm{~h}$ after sepsis induction. Inflammation and bacterial clearance were assessed in PLF, BAL, and organs of the septic animals.

Results: Phagocytic efficacy and bacterial killing capacities were significantly increased in $M \phi$ from both healthy donors and septic patients co-cultured with UCMSCs than in control M $\phi$. M $\phi$ exposed to MSCs expressed more $\mathrm{HO}-1$, an enzyme with anti-inflammatory and bactericidal properties. Peritoneal macrophages isolated from animals subjected to abdominal fecal demonstrated greater phagocytosis of SOZ when taken from UC-MSC treated vs. vehicle treated animals. Macrophages also expressed more $\mathrm{HO}-1$. The direct $\mathrm{HO}-1$ inducer Hemin increased macrophage phagocytosis from vehicle but not from UC-MSC treated animals. In contrast, the HO-1 antagonist ZnPP decreased phagocytosis in M $\phi$ from UC-MSC treated animals to the levels seen in macrophages from vehicle treated animals. The HO-1 increase in $\mathrm{M} \phi$ was dependent on pro-resolvin mediators secreted by UC-MSCs, specifically PGE2 and Lipoxin A4. Septic animals cleared bacteria and recovered faster, if treated with UC-MSCs.

Conclusions: UC-MSCs are promising tool for sepsis treatment. UC-MSCs enhance macrophage phagocytosis and increase bacterial clearance, in part, via a mechanism involving UC-MSC induced $\mathrm{HO}-1$ expression in macrophages.

\section{References}

1. Seymour CW, Liu VX, Iwashyna TJ, et al.: JAMA, 315(8):762, 2016.

2. Keane C, Jerkic M, and Laffey JG: Anesthesiology, 127(6):1017-1034, 2017.

3. Curley GF, Jerkic MJ, Dixon S, et al: CCM, 45(2):e202-e212, 2017.

4. Curley GF, Hayes M, Ansari B, et al: Thorax 67(6):496, 2012. 
5. Gupta N, Krasnodembskaya A, Kapetanaki M, et al: Thorax 67(6):533, 2012.

6. McIntyre LA, Stewart DJ, Mei SHJ et al: AJRCCM, 197(3):337-347, 2018. 
Moving to a Newly Built Adult Intensive Care Unit with Single Patient Rooms: Impact on Clinicians and ICU Performance

Laporta, Denny ${ }^{1,2}$; Boitor, Madalina Boitor ${ }^{3,4}$; Gélinas, Céline ${ }^{3,4}$.

1 Adult Critical Care, Jewish General Hospital, Montreal, Quebec, Canada

2 Faculty of Medicine, McGill University, Montreal, Quebec, Canada

3 Ingram School of Nursing, McGill University, Montreal, Quebec, Canada

4 Centre for Nursing Research and Lady Davis Institute, Jewish General Hospital,

Montreal, Quebec, Canada

Introduction: The adult intensive care unit (ICU) of the Jewish General Hospital (JGH) moved from a multi-patient room (MPR) to a single patient room (SPR) environment in 2016. The new adult SPR-ICU was built based on recent ICU design guidelines with the goal to create a healing environment.

Objectives: To compare ICU clinicians' work stress, satisfaction, obstacles to work performance, team functioning, ability to provide family-centered care, with unit performance in an adult open ward to a new SPR-ICU.

Methods: Design: A pre/post-test design was used for this study. Setting: This study was conducted at the ICU of the JGH. Prior the move, the ICU had a centralized nursing station and 22 beds of which 14 were in open areas, 6 were SPR and one was a double-patient room. After the move, the ICU had 36 beds all in SPRs with miniworkstations outside all patient rooms and decentralized nursing stations. Natural light is maximized through extra-large windows in all patient rooms. Sample: Clinicians were eligible for participation if they were full- or part-time ICU employees. Temporary clinicians, students, medical residents, fellows and newly hired staff on orientation were excluded. Eligible clinicians willing to participate provided written informed consent and were asked to complete a questionnaire package prior to and 12 months after the move to the new SPR-ICU.

Results: A total of 83 clinicians (51 nurses, 21 respiratory therapists, 4 physicians, 4 physiotherapists and 3 orderlies) with an average of 10 years of ICU experience completed the questionnaires before and 60 of them at 12 months after the move. Clinicians perceived higher work stress (on a 1-4 scale) after the move (mean=2.13, $\mathrm{SD}=0.52)$ compared to before (mean $=1.92, \mathrm{SD}=0.48)(\mathrm{t}(58)=4.95, \mathrm{p}<0.001)$, and less job satisfaction (on a 1-5 scale) after (mean $=3.47, \mathrm{SD}=0.65)$ than before the move ( mean=3.69, $S D=0.47)(t(58)=2.70, p=0.009)$. Whereas clinicians reported experiencing more obstacles in performing tasks after the move, the use of technology and tools improved in the new SPR-ICU $(p<0.05)$. The new SPR-ICU was associated with less collaborative work (on a 1-7 scale) (mean $=5.11, \mathrm{SD}=0.83$ ) compared to the old ICU (mean=5.62, SD=0.76) (t $(58)=4.45, p<0.001)$ in terms of communication, implicit coordination and synchrony. Clinicians did not perceive significant changes in their ability to provide family-centered care with the move to the SPR-ICU (t $(58)=0.46$, $p=0.645)$, but believed that environmental noise was reduced $(t(58)=5.34, p<0.001)$. The degree to which patient rooms were organized was perceived to remain unchanged $(\mathrm{t}(58)=0.44, \mathrm{p}=0.659)$. During these same time periods, a $7 \%$ increase in ICU admissions was noted. Review of ICU performance indicators revealed the following trends: an increase in emergency admissions (medical and surgical) and in elderly (age $>80$ ) patients, and a decrease in ICU readmissions, VAP and hospital length of stay. Severity scores and mortality were unchanged.

Conclusions: The new SPR-ICU was perceived to reduce obstacles in using technology and tools, and to decrease environmental noise, but was associated with more difficulty in working collaboratively, higher work stress and less job satisfaction. Whether clinicians' perceptions were also influenced by changing clinical demands remains unanswered, but the resulting ICU performance indicators over this period remained the same or favorably improved. 
Table 1 - Description of Participating ICU Clinicians

\begin{tabular}{|c|c|c|}
\hline Variable & $\begin{array}{c}\text { Pre-move } \\
(\mathrm{n}=83)\end{array}$ & $\begin{array}{c}12 \text { months } \\
\text { post-move } \\
(n=60)\end{array}$ \\
\hline \multicolumn{3}{|l|}{ Mean (SD) } \\
\hline Age, in years & $38.7(9.9)$ & $40.5(10.2)$ \\
\hline $\begin{array}{l}\text { Years of professional service at the } \\
\text { study site }\end{array}$ & $11.8(8.8)$ & $13.2(8.9)$ \\
\hline Years of experience in the ICU & $10.0(8.4)$ & $11.2(8.8)$ \\
\hline Number of hours worked per week & $37.1(9.72)$ & $36.7(10.2)$ \\
\hline \multicolumn{3}{|l|}{ N (\%) } \\
\hline Profession: Nurse & $51(61.4)$ & $38(63.3)$ \\
\hline & $4(4.8)$ & $2(3.3)$ \\
\hline $\begin{array}{ll}\text { Physician } \\
\text { Respiratory therapist }\end{array}$ & $21(25.3)$ & $16(26.7)$ \\
\hline Physiotherapist & $4(4.8)$ & $3(5.0)$ \\
\hline Orderly & $3(3.6)$ & $1(1.7)$ \\
\hline \multirow[t]{2}{*}{ Gender: $\quad$ Female } & $64(77.1)$ & $49(81.7)$ \\
\hline & $19(22.9)$ & $11(18.3)$ \\
\hline Education: Collegial diploma & $25(30.1)$ & $18(30.0)$ \\
\hline BA (Bachelor's degree) & $35(42.2)$ & $31(51.7)$ \\
\hline MA (Master's degree) & $8(9.6)$ & $9(15.0)$ \\
\hline Doctorate & $3(3.6)$ & $2(3.3)$ \\
\hline \multicolumn{3}{|l|}{ Employment status: } \\
\hline Permanent full time & $24(28.9)$ & $49(81.7)$ \\
\hline Permanent part time & $7(8.4)$ & $9(15.0)$ \\
\hline Temporary full time & $1(1.2)$ & $1(1.7)$ \\
\hline Other & $1(1.2)$ & $1(1.7)$ \\
\hline
\end{tabular}

Table 2 - Means and SDs of Scores Reported by Clinicians on the Various Outcomes

\begin{tabular}{|c|c|c|c|c|}
\hline Outcomes & $\begin{array}{c}\text { Range of scores } \\
\text { (meaning) }\end{array}$ & $\begin{array}{c}\text { Pre-move } \\
\text { (n=83) }\end{array}$ & $\begin{array}{c}12 \text { months } \\
\text { post-move } \\
(\mathrm{n}=60)\end{array}$ & $\begin{array}{c}\text { RM-ANOVA } \\
\text { p value }\end{array}$ \\
\hline $\begin{array}{c}\text { Performance Obstacles } \\
\text { Questionnaire (POQ) }\end{array}$ & $\begin{array}{c}1 \text { to } 5 \\
\text { (higher perception of obstacle) }\end{array}$ & $2.59(0.47)$ & $2.59(0.48)$ & 0.063 \\
\hline Tasks & & $3.21(0.79)$ & $3.52(0.82)$ & 0.016 \\
\hline Technology and tools & & $2.89(0.57)$ & $2.39(0.71)$ & $\mathrm{P}<0.001$ \\
\hline Environmental noise & & $3.78(1.08)$ & $3.39(0.98)$ & $\mathrm{P}<0.001$ \\
\hline $\begin{array}{c}\text { Nurse Stress Scale } \\
\text { (NSS) }\end{array}$ & $\begin{array}{c}1 \text { to } 4 \\
\text { (more stress) }\end{array}$ & $1.92(0.48)$ & $2.13(0.52)$ & $\mathrm{P}<0.001$ \\
\hline $\begin{array}{c}\text { Collaborative Work } \\
\text { Questionnaire }\end{array}$ & $\begin{array}{c}1 \text { to } 7 \\
\text { (better collaboration) }\end{array}$ & $5.62(0.76)$ & $5.11(0.83)$ & $\mathrm{P}<0.001$ \\
\hline Communication & & $5.75(0.80)$ & $5.18(0.92)$ & $\mathrm{P}<0.001$ \\
\hline Implicit coordination & & $5.50(0.89)$ & $5.01(1.01)$ & $\mathrm{P}<0.001$ \\
\hline Synchrony & 1 to 5 & $3.78(0.70)$ & $5.25(0.90)$ & $\mathrm{P}<0.001$ \\
\hline $\begin{array}{c}\text { Job Satisfaction Scale } \\
\text { (JSS) }\end{array}$ & (better satisfaction) & $3.69(0.47)$ & $3.47(0.65)$ & 0.019 \\
\hline Quality of care & & $3.03(0.68)$ & $2.83(0.82)$ & 0.026 \\
\hline Time to do job & & & \\
\hline
\end{tabular}

"Standard score = (reported score-1)/tool score range x100 (Illfeld, F.W., 1976). 


\section{References}

1. Thompson, D. R., Hamilton, K., Cadenhead, C. D., Swoboda, S. M., Schwindel, S. M., Anderson, D. C. et al. (2012). Guidelines for intensive care unit design. Critical Care Medicine, 40(5), 15861600.

2. Gurses, A. P., \& Carayon, P. (2009). Exploring performance obstacles of intensive care nurses. Applied Ergonomics, 40(3), 509-518.

3. Mitchell, M. L., Cert, G., Burmeister, E., Chaboyer, W. \& Shields, L. (2012). Psychometrics of the "Family-Centred Care Survey - Adult Scale". The International Journal of Person Centered Medicine, 2(4), 792-798.

4. Gray-Toft, P. \& Anderson, J. G. (1981). Stress among hospital nursing staff: Its causes and effects. Journal of Social Science Medicine, 15A, 639-647

5. Chiocchio, F., Grenier, S, O'Neill, T.A., Savaria, K, \& Willms, D.J. (2012). The effects of collaboration on performance: A multilevel validation in project teams. International Journal of Project Organisation and Management, 4(1), 137. Rousseau, V., Aubé, C., \& Savoie, A. (2006). Le fonctionnement interne des équipes de travail : conception et mesure. Revue canadienne des sciences du comportement, 38, 120-135.

6. Hinshaw, A.S., \& Atwood, J.R. (1985). Anticipated turnover among nursing staff. Final Report. The University of Arizona, Tucson.

7. Ilfeld, F.W. (1976). Further validation of a psychiatric symptom index in a normal population. Psychological Reports, 39, 1215-1228. 


\section{Optimizing Mobility for Critically III Patients Undergoing Continuous Renal} Replacement Therapy: A Retrospective Cohort Study (MOvE CRRT)

Trumble, Drayton E ${ }^{1}$; DeVries, Jodi²; Reil, Ellen²; Wang, Xiaoming ${ }^{3} ;$ Bagshaw, Sean M 1,2; Rewa, Oleksa ${ }^{1,2}$

1 Department of Critical Care Medicine, Faculty of Medicine and Dentistry, University of Alberta, Edmonton, Alberta, Canada

2 Department of Critical Care Medicine, General Systems Intensive Care Unit, University of Alberta Hospital, Alberta Health Services, Edmonton, Alberta, Canada 3 Research Facilitation, Alberta Health Services, Edmonton, Alberta, Canada

Introduction: Critically ill patients admitted to the intensive care unit (ICU) requiring continuous renal replacement therapy (CRRT) can suffer from decreased mobility interventions due to fear of adverse events. Physical therapy (PT) interventions have been shown to be feasible and safe in patients undergoing CRRT; however, there are few published recommendations or protocols ${ }^{1}$. This warrants a baseline audit of PT interventions in those receiving CRRT to help guide future mobilization practices in these patients.

Objectives: Our primary objective was to describe the incidence of PT interventions in patients receiving CRRT. Additionally, we sought to highlight possible barriers to treatment, identify any adverse events, and prepare recommendations for future protocols aimed at increasing PT interventions in ICU patients receiving CRRT. Methods: We conducted a retrospective observational cohort study of patients over the age of 18 years admitted to the General Systems ICU at the University of Alberta Hospital between April 1, 2015, and April 1, 2017. Any patient with a length of stay (LOS) greater than 7 days and CRRT treatment for greater than 72 hours was considered. We generated a matched cohort with similar age, admission criteria, and acuity of illness. Data collection was conducted via a focused audit of our clinical information system (CIS). Data were extracted on socio-demographics, diagnoses, chronic diseases, illness severity, and treatment intensity. PT data was collected from the repository's physiotherapy charting and included treatment delays, adverse events, and the highest level of mobilization achieved. Data analysis was primarily descriptive. Data was collected from any PT sessions between initiation and discontinuation of CRRT, regardless of whether CRRT was running during the specific PT intervention. Results: Ninety-two patients fit our criteria, of which 50 were randomly selected as cases. The median age was 55 years, 27 (54\%) were male, and median admission APACHE II and GCS scores were 30 and 12.5 respectively. The median total CRRT time was 141 hours. No patients died while in ICU, however, $10(20 \%)$ died in hospital. These patients were matched with 51 similar control patients. No adverse events during physiotherapy were reported. CRRT line access during physiotherapy was a common concern of PTs or RNs, and limited or postponed treatment in 7 (14\%) patients. Femoral dialysis catheters were most often listed as the site of concern. For CRRT patients, the highest level of mobility achieved was ambulation in $1(2 \%)$, active in $17(34 \%)$, passive in $13(26 \%)$, and none in $19(38 \%)$. In our 51 matched non-CRRT patients, the highest level of mobility achieved during their entire ICU admission was ambulatory in $33(65 \%)$, active in $10(20 \%)$, passive in $2(4 \%)$, none in $4(8 \%)$, and incomplete data in $2(4 \%)$. Thirteen (26\%) CRRT patients had an interruption of their PT program of at least 1 day for medical reasons, compared to $22(43 \%)$ in the matched controls. Four (8\%) of the CRRT patients had their PT program delay attributed to CRRT.

Conclusion: PT interventions in ICU patients undergoing CRRT continues to appear feasible and safe. CRRT patients were mobilized to a lesser degree than a matched cohort of similar illness severity. Once started, PT schedules were interrupted less often in CRRT patients than in controls. A protocol defining PT interventions in this population, ensuring to highlight line concern recommendations, is needed. 


\section{References}

1. Feasibility and Safety of Physical Therapy during Continuous Renal

Replacement Therapy in the Intensive Care Unit. Toonstra AL, Zanni JM, Sperati

CJ, Nelliot A, Mantheiy E, Skinner EH, Needham DM. Ann Am Thorac Soc. 2016 May;13(5):699-704. 
Organ and Tissue Donation Physician Leadership Model: Mapping a Way Forward

Sarti, Aimee $^{1}$; Sutherland, Stephanie ${ }^{1}$; Landriault, Angele ${ }^{2}$; Healey, Andrew ${ }^{3}$; Dhanani, Sonny ${ }^{4}$; Cardinal, Pierre ${ }^{1}$

1 Department of Critical Care, Ottawa Hospital, Ottawa, Ontario, Canada

2 Royal College of Physicians and Surgeons of Canada (RCPSC), Practice, Performance and Innovation (PPI) unit, Ottawa, Ontario, Canada

3 Division of Emergency Medicine, Department of Medicine, McMaster University, Hamilton, Ontario, Canada

4 Department of Pediatrics, University of Ottawa, Children's Hospital of Eastern Ontario (CHEO), Ottawa, Ontario, Canada

Introduction: In 2015 the Trillium Gift of Life Network (TGLN) began enhancing the organ and tissue donation program in Ontario by appointing specially trained critical care physicians to support hospitals as they integrate donation into quality end of life care and work towards changing hospital culture. To understand the level of implementation, measure the degree of communication and collaboration within the physician leadership model, and better understand physician leadership and engagement practices at the hospital level we constructed a series of social network maps.

Methods: In 2016 we employed a quantitative social network design and sent surveys to all five Regional Medical Leads (RMLs) and all 52 Hospital Donation Physicians (HDPs) in Ontario to better understand their roles, practice context, and to map their social networks. Social network analysis was performed with raw survey data with NVIVO software to produce a map of Ontario's physician leadership model as well as individual maps for each hospital-based physician.

Results: The social network maps included a sociogram of the entire network as well as maps comparing the five RML peer learning networks (i.e., RML connectivity to HDPs at the hospital level). We were able to illustrate the key local opinion leaders / social influencers at each of the hospitals. Also, a central part of the survey was to ascertain the current state of the HDP social network in terms of knowledge sharing and support. Many respondents viewed the implementation physician leadership and engagement roles as central facilitators of TGLNs vision in Ontario. At the same time, respondents felt that the implementation of the roles was problematic in that these roles were not clearly defined nor locally communicated to hospitals at the outset. HDPs provided varied answers as to what they perceived their role to be, the most frequently described role was to monitor performance/quality improvement.

Conclusion: Social network mapping of the physician leadership model provides a better understanding of the functioning, and ongoing peer learning that occurs within the network. This kind of social network mapping also permits an ongoing evaluation of the network over time by tracking central information brokers' network positions. 
Organ Donation Programs and the Definition of Success: A Scoping Review Preliminary Results

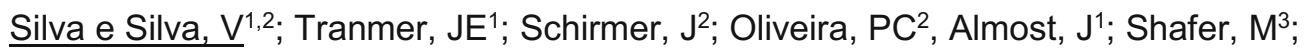
Dhanani, $\mathrm{S}^{4}$; Roza, BA ${ }^{2}$

1 School of Nursing, Queen's University, Kingston, Ontario, Canada

2 School of Nursing, Federal University of Sao Paulo, Sao Paulo, Brazil

3 Department of Sociology, University of Toronto, Toronto, Ontario, Canada

4 Department of Medicine, University of Ottawa, Ottawa, Ontario, Canada

Introduction: The effectiveness of organ donation program performance has been predominantly evaluated through cross-country comparisons of quality indicators and legislative processes. However, the variation of performance within and among programs remains poorly explained and understood.

Objectives: This scoping review aims to synthesize the current evidence on key organizational attributes and processes of international organ donation programs associated with successful outcomes.

Methods: We followed a systematic scoping review protocol in accordance with the Joanna Briggs Institute. This included a detailed search of appropriate databases (PUBMED, CINAHL, EMBASE, LILACS, ABI Business ProQuest, Business Source Premiera) and grey literature (Organ donation association websites, Google Scholar (first eight pages), and Research Gate) and the reference list of all identified documents was scanned. Inclusion criteria: Studies, reports or documents that investigated and provided measures for organ donation program, processes, or initiatives effectiveness/success worldwide (conversion rates, number of organs transplanted, cost-effective measures); studied program evaluation generically, but contains success measures and definitions for programs; definitions of organ donation program effectiveness; measurement of good processes or organ donation program improvements. Full text available in Spanish, Portuguese or English, with no year limit. Exclusion criteria: Studies not related to the 'concept of interest' (success, effectiveness, good processes description); evaluation of other programs (organ transplant programs, tissue donation and transplantation programs, mental health programs, etc.); other healthcare and non-healthcare related studies that do not contain definitions or measures for effectiveness and success in organ donation program evaluation; conference abstracts not linked to full text article. non-heart beating/donation after circulatory death programs; articles about knowledge and attitude of people about organ donation; tissue donation programs (bone marrow, skin, bones, eggs, sperm, stem-cell, cornea); paired kidney donation programs and living donation programs; composite tissue transplant (face/hand/leg); organ donation quality indicators for organ transplantation and not focused in the organ donation

programs. All documents are being reviewed by two independent reviewers to screen: (1) studies selection by title and abstract reading (in progress), and (2) study selection after full text reading. The synthesis will include quantitative analysis (e.g., frequency analysis) of general data on the type of study, country of study, terminologies used to describe "success", etc.; and qualitative analysis (e.g., content analysis) of the components of the research purpose and conceptual definitions of "success" in organ donation programs.

Results: We have identified 7832 articles, 958 duplicates were removed, 3610 articles were screened and 1755 were excluded. Data extraction is done and we have identified three main themes: barriers to success, success factors and route to success. The themes will be further explored and the anticipated date of completion is December, 2018. 
Conclusion: The outcomes of this study will unfold the current meaning of "success" for the scientific community in organ donation and transplantation programs worldwide and inform the development of quality initiatives in organ donation programs. 
Post-Mortem Expressions of Compassion: A Secondary Qualitative Analysis of Family and Clinician Experiences of Sympathy Cards in the 3 Wishes Project

Takaoka, Alyson ${ }^{1}$; Clarke, France ${ }^{1}$; Hoad, Neala ${ }^{2}$; Swinton, Marilyn ${ }^{1}$; Goksoyr, Sophia ${ }^{3}$; Leblanc, Allana ${ }^{3}$; Foster Denise ${ }^{3}$; Lockington, Julie ${ }^{3}$; Dhingra, Vinay ${ }^{3}$; Neville, Thanh"; Xu, Xueqing ${ }^{5}$; Kao, Yuhan ${ }^{5}$; Phung, Peter ${ }^{6}$; Smith, Orla ${ }^{7}$; Vanstone, Meredith ${ }^{8}$; Cook, Deborah ${ }^{1,9}$

1 Department of Health Research Methods, Evidence and Impact, McMaster University, Hamilton, Ontario, Canada

2 Critical Care Research Department, St. Joseph's Healthcare, Hamilton, Ontario, Canada

3 Department of Critical Care Medicine, Vancouver General Hospital, Vancouver, Ontario, Canada

4 Department of Medicine/Division of Pulmonary \& Critical Care, University of California Los Angeles, Los Angeles, United States of America

5 Department of Nursing, University of California Los Angeles, Los Angeles, United States of America

6 Department of Medicine/Division of Palliative Care, University of California Los Angeles, Los Angeles, United States of America

7 Critical Care Department, St. Michael's Hospital, Toronto, Ontario, Canada

8 Department of Family Medicine, McMaster University, Hamilton, Ontario, Canada

9 Department of Medicine, McMaster University, Hamilton, Ontario, Canada

Introduction: Expressions of sympathy from clinicians may be welcome for grieving family members of patients who die in the intensive care unit (ICU). However, a recent randomized controlled trial evaluating a semi-structured condolence letter in France suggested increased symptoms of depression and post-traumatic stress amongst recipients ${ }^{1}$.

Objectives: To explore clinician and family member experiences of sending or receiving hand-written sympathy cards after death in the ICU nested within the 3 Wishes Project which seeks to honour patients at the end of life. This clinical and research program originating at St Joseph's Healthcare Hamilton (SJH) is also operating at Vancouver General Hospital (VGH), St. Michael's Hospital in Toronto and Ronald Reagan UCLA Medical Center in the United States.

Methods: A core component of the 3 Wishes Project is an invitation for nurses, physicians, physiotherapists and respiratory therapists- both staff and trainees alike- to write a few words to bereaved family members in a sympathy card, mailed 1-2 weeks after each death. For this analysis, we searched a qualitative database of 200 clinician and family interview transcripts. Data related to sympathy cards were independently coded by 2 investigators using conventional content analysis and a list of codes was developed by consensus.

Results: We identified 27 transcripts from individual interviews ( $n=14$ family members, $\mathrm{n}=11$ clinicians) and 2 focus groups ( $\mathrm{n}=8$ clinicians) in which sympathy cards sent by the 3 Wishes Program were discussed in 3 participating centers (SJH, VGH, UCLA). In $16(59.3 \%)$ transcripts, participants spontaneously discussed sympathy cards. In 11 $(40.7 \%)$ transcripts, participants were explicitly asked to reflect on their experiences sending or receiving sympathy cards. Family members felt deeply touched by personalized messages in the cards and saw them as a heartfelt act of compassion by the ICU team. The cards were a welcome surprise and offered an appreciated connection to clinicians with whom they had a relationship. Families reported feeling warmly remembered and they were eager to tell their relatives, friends and other hospital staff about receiving the card. Clinicians viewed the cards as an opportunity to offer expressions of their shared humanity with families, reminding them that they and their loved one were not forgotten. Most staff were keen to write a few words to families of patients they cared for who they knew well enough to authentically express their 
sorrow. While some staff preferred to pen a message only if they directly cared for the patient, others were motivated to offer sentiments of condolence if they had a brief interaction with the patient or knew them indirectly through seeing their family in the ICU. Taking the time to discuss memories of a deceased patient in the company of a colleague offered an opportunity for reminiscing and closure. A unifying concept was that both family members and clinicians experienced sympathy cards as an important continuation of care extended to family members of deceased ICU patients. Sympathy cards symbolized a special post-mortem connection for both parties.

Conclusions: Family members found sympathy cards to be a meaningful, compassionate gesture by the ICU team. Inviting staff who cared for deceased patients to offer personalized condolences in a card mailed to bereaved family members may foster sincere, valued expressions of sympathy when individualized and unstructured, as contextualized in the 3 Wishes Program.

\section{References}

1. Kentish-Barnes, N., Chevret, S., Champigneulle, B., Thirion, M., Souppart, V., Gilbert M. et al. (2017). Effect of a condolence letter on grief symptoms among relatives of patients who died in the ICU: a randomized clinical trial. Intensive Care Medicine. 43: 473-484 
Practice Patterns of Phenobarbital Use in Severe Alcohol Withdrawal Syndrome

Buell, Danielle ${ }^{1}$; Filewod, Niall'2; Ailon, Jonathon ${ }^{3}$; Burns, Karen E. A. ${ }^{2}$

1 Department of Internal Medicine, University of Toronto, Toronto, Ontario, Canada

2 Department of Critical Care, St. Michael's Hospital, University of Toronto, Toronto, Ontario, Canada

3 Department of General Internal Medicine, St. Michael's Hospital, University of Toronto, Toronto, Canada

Introduction: Treatment of acute alcohol withdrawal syndrome (AAWS) with symptom-triggered benzodiazepine therapy is well described1,2. However, the optimal management of patients with refractory or severe AAWS (sAAWS) is unclear. Three small randomized control trials suggest that intravenous phenobarbital may be an effective adjuvant therapy for the treatment of SAAWS3-5. Despite this, several factors likely limit its use in clinical practice.

Objectives: To characterize current practice patterns in treatment of sAAWS and assess the current attitudes and knowledge around the use of phenobarbital for patients with sAAWS at centers specializing in inner-city healthcare.

Methods: We conducted a cross-sectional multi-center, interdisciplinary, selfadministered paper and electronic survey 6 of general internists, intensivists, psychiatrists and emergency medicine physicians at St. Michael's (ON) and St. Paul's Hospital (BC).

Results: We received $118 / 194$ (61\% response rate) completed questionnaires. Most respondents were male (62\%), older than $40(76 \%)$, in practice for a mean of 14 years, with $66 \%$ of their time spent in clinical practice. Only 3 respondents were addiction specialists, 2 of whom used phenobarbital in their practice. Respondents managed an average of 32 cases of AAWS in a 6-month period, of which $7(22 \%)$ were severe. Respondents defined severe alcohol withdrawal by the presence of seizures $(80 \%)$, hallucinations $(60 \%)$ and a CIWA score $>15$ after $60 \mathrm{mg}$ of diazepam $(56 \%)$. Most respondents $(60.4 \%)$ did not use phenobarbital in their practice. Of those that used phenobarbital, $58 \%$ considered using it as an adjuvant treatment if patients were refractory to symptom-based benzodiazepine therapy, whereas others waited until seizures developed (10\%) or ICU admission was warranted (18\%). Intravenous administration was twice as likely to be used as oral phenobarbital. Most respondents $(58 \%)$ did not know the peak onset of phenobarbital activity, $33 \%$ were unfamiliar with the side effects, and $42 \%$ were uncomfortable with its contraindications. Only $6 \%$ of respondents were aware of the evidence supporting phenobarbital use. Haloperidol and clonidine were the 2 most commonly used adjuvant treatments used to treat sAAWS. Although $59 \%$ of respondents were uncomfortable using phenobarbital in their practice, $85 \%$ were either comfortable or neutral with enrolling patients in a trial to investigate the use of phenobarbital for patients with sAAWS.

Conclusion: Our results suggest that despite seeing a high volume of sAAWS, there is considerable stated practice variation in how clinicians treat patients with sAAWS. Our results highlight both a lack of awareness regarding the current evidence supporting phenobarbital use for sAAWS and discomfort with its use in practice. Those that used phenobarbital, reported using the IV preparation and administering it early in treatment. Most respondents felt that greater evidence was needed, and expressed equipoise for enrolling patients with sAAWS in a trial evaluating IV phenobarbital as an adjuvant therapy to symptom-triggered benzodiazepine therapy. 
Figure 1 - Summary of responses from All Specialties to Likert Scale Questions; Mean Indicated by Vertical Line

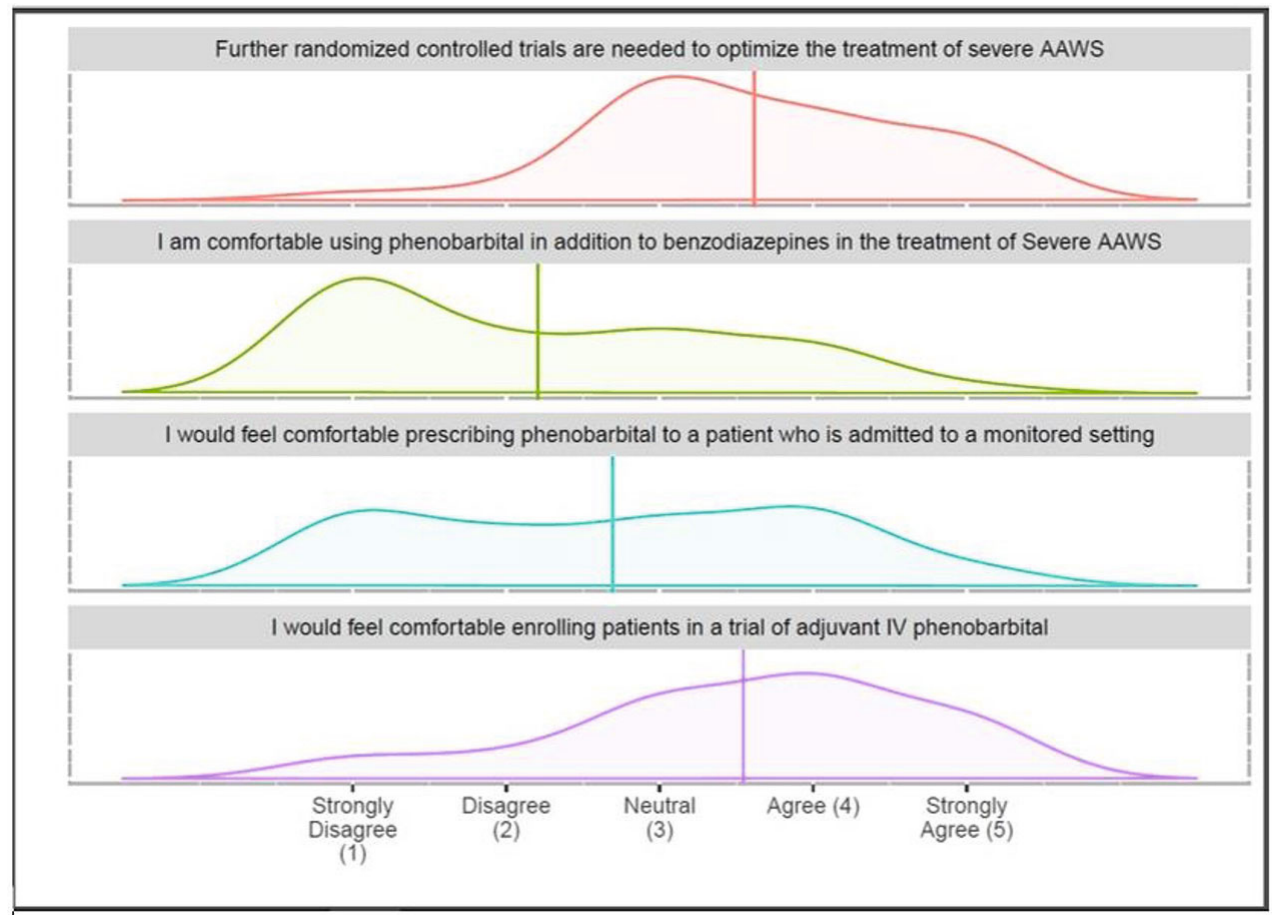

Figure 2 - Number of Respondents Who Reported Using Each Adjuvant Therapy to Manage Severe AAWS (Total Respondents=101)

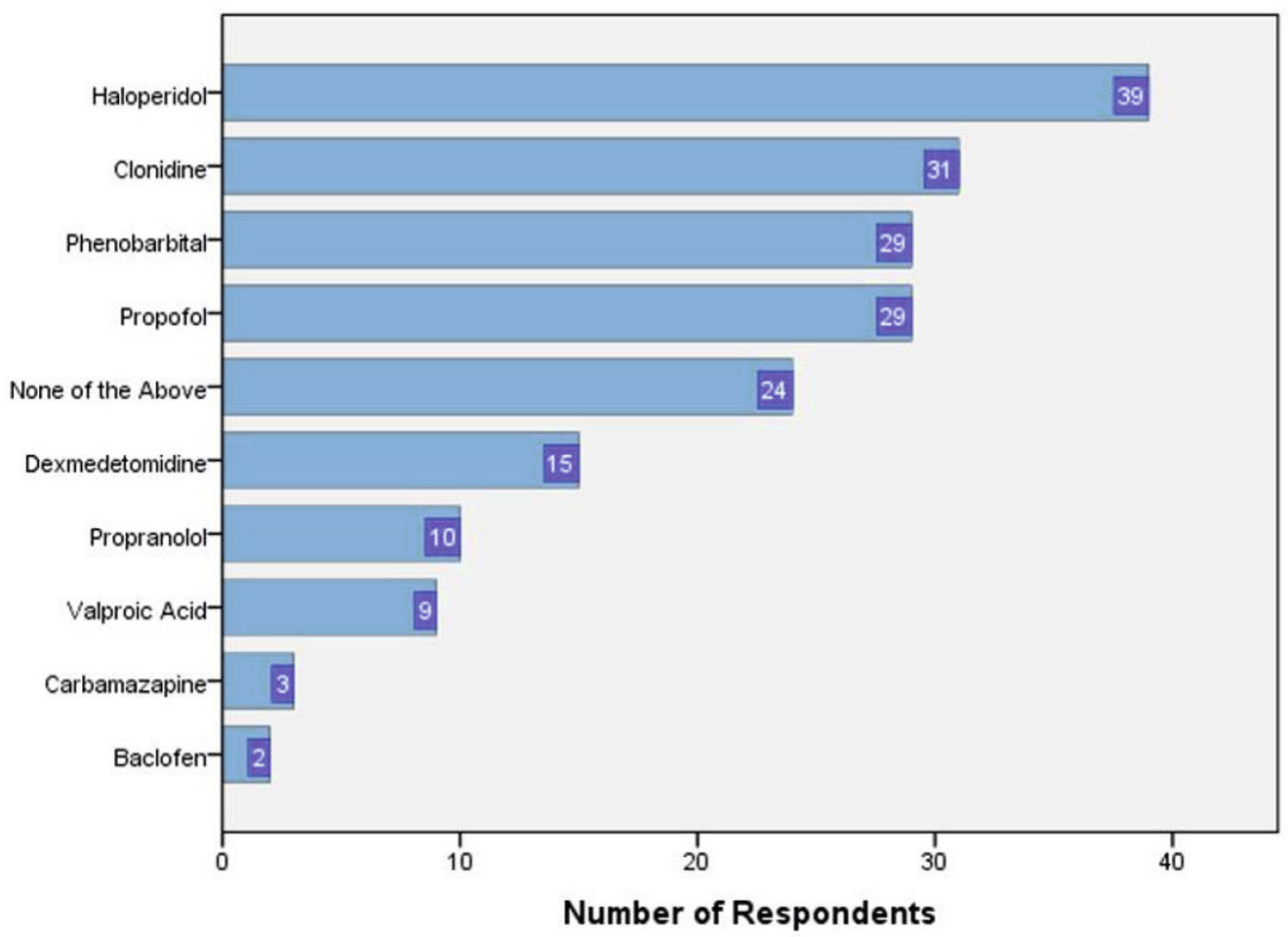


Table 1 - By specialty and total; mean number of AAWS cases seen, mean number of severe AAWS cases seen, mean years in practice and mean proportion of practice clinical duties, denoted if statistically significantly different from the combined mean ${ }^{*}=p<0.05,{ }^{* *} p<0.01$

\begin{tabular}{|c|c|c|c|c|c|}
\hline \multirow{8}{*}{ Specialty } & & $\begin{array}{c}\text { \# of Severe } \\
\text { AAWS per } 6 \\
\text { months }\end{array}$ & $\begin{array}{c}\text { \# of AAWS } \\
\text { per } 6 \text { months }\end{array}$ & $\begin{array}{l}\text { Years In } \\
\text { practice }\end{array}$ & $\begin{array}{c}\text { Proportion of } \\
\text { practice } \\
\text { clinical (\%) }\end{array}$ \\
\hline & Combined mean & 6.9 & 31.5 & 14.4 & 67.0 \\
\hline & Internal Medicine & 6.6 & $22.1^{*}$ & 14.3 & 58.0 \\
\hline & Psychiatry & 4.2 & $18.7^{*}$ & $10.2^{*}$ & 78.4 \\
\hline & Critical Care & 7.2 & $14.8^{\star *}$ & 15.1 & $42.3^{* *}$ \\
\hline & Emergency Medicine & 8.5 & $48.9^{* *}$ & 15.5 & 74.4 \\
\hline & General Surgery & 3.8 & $9.5^{*}$ & 17.0 & 47.5 \\
\hline & Anesthesiology & $0.8^{* *}$ & $3.2^{* *}$ & 18.3 & $83.3^{*}$ \\
\hline
\end{tabular}

\section{References}

1. Saitz R, Friedman LS, Mayo-Smith MF. Alcohol withdrawal: a nationwide survey of inpatient treatment practices. J Gen Intern Med. 1995 Sep 1;10(9):479-87.

2. Ward D, Murch N, Agarwal G, Bell D. A multi-centre survey of inpatient pharmacological management strategies for alcohol withdrawal. QJM. 2009.

3. Rosenson J, Clements C, Simon B, Vieaux J, Graffman S, Vahidnia F, et al. Phenobarbital for acute alcohol withdrawal: a prospective randomized double-blind placebo-controlled study. J Emerg Med. 2012 Sep 19;44(3):592-598.e2.

4. Hendey GW, Dery RA, Barnes RL, Snowden B. A prospective, randomized, trial of phenobarbital versus benzodiazepines for acute alcohol withdrawal. The American journal of emergency medicine 2011;29(4):382.

5. Kramp P, Rafaelsen OJ. Delirium tremens: a double-blind comparison of diazepam and barbital treatment. Acta Psychiatr Scand. 1978 Aug 1;58(2):174-90.

6. Burns KEA, Duffett M, Kho ME, Meade MO, Adhikari NKJ, Sinuff T, et al. A guide for the design and conduct of self-administered surveys of clinicians. CMAJ. $2008 \mathrm{Jul}$ $30 ; 179(3): 245-52$ 
Pre-Arrest and Intra-Arrest Prognostic Factors for Survival to Discharge in InHospital Cardiac Arrest - A Systematic Review and Meta-analysis

Fernando, Shannon M. ${ }^{1,2}$; Tran, Alexandre ${ }^{3,4}$; Cheng, Wei5; Rochwerg, Bram ${ }^{6,7}$; Taljaard, Monica ${ }^{4,5}$; Kyeremanteng, Kwadwo ${ }^{1,5}$; Mclsaac, Daniel I.4,5,8; Perry, Jeffrey $\mathrm{J.}, 2,4,5$

1 Division of Critical Care, Department of Medicine, University of Ottawa, Ottawa, Ontario, Canada

2 Department of Emergency Medicine, University of Ottawa, Ottawa, Ontario, Canada

3 Department of Surgery, University of Ottawa, Ottawa, Ontario, Canada

4 School of Epidemiology and Public Health, University of Ottawa, Ottawa, Ontario,

Canada

5 Clinical Epidemiology Program, Ottawa Hospital Research Institute, Ottawa, Ontario, Canada

6 Department of Medicine, Division of Critical Care, McMaster University, Hamilton, Ontario, Canada

7 Department of Health Research Methods, Evidence, and Impact, McMaster

University, Hamilton, Ontario, Canada

8 Department of Anesthesiology and Pain Medicine, University of Ottawa, Ottawa, Ontario, Canada

Introduction: Prognosis from adult in-hospital cardiac arrest (IHCA) is typically poor, with roughly $10-20 \%$ of patients surviving to hospital discharge. Likelihood of survival with good neurologic function is even lower. Therefore, it is important for clinicians to understand the factors influencing survival from IHCA, to provide accurate information to patients for informed goals-of-care discussions, and to consider cessation of inhospital resuscitation when the likelihood of survival is exceedingly low.

Objectives: We sought to summarize all studies evaluating pre-arrest and intra-arrest prognostic factors associated with survival in adult patients with IHCA.

Methods: We searched six databases (including Medline, EMBASE, and Web of Science) from inception through May 30, 2018. We included English-language studies (including observational studies, randomized controlled trials, and non-randomized controlled trials) evaluating prognostic factors associated with survival to hospital discharge in adult patients with IHCA. We excluded studies of patients with intraoperative arrest, and studies which only included patients who achieved return of spontaneous circulation (ROSC). We followed PRISMA guidelines and the Cochrane Handbook for Systematic Reviews of Prognosis. Two reviewers independently extracted data and assessed study quality using the Ottawa-Newcastle Scale. The main outcome was survival to hospital discharge. Pooled adjusted odds ratios (OR) were calculated. All adjusted ORs included in meta-analysis had to at minimum adjust for age and sex.

Results: The initial search yielded 8,856 articles. Following screening and full-text evaluation, 51 studies were deemed eligible and were included. We found that patients with existing comorbidities such as active malignancy (pooled adjusted OR 0.57 [95\% Cl: 0.45-0.71]) and chronic kidney disease (pooled adjusted OR 0.62 [95\% Cl: 0.440.89]) had significantly decreased odds of survival. Likewise, patients with an admission diagnosis of sepsis had significantly decreased odds of survival (pooled adjusted OR 0.80 [95\% Cl: 0.70-0.91]). Factors associated with significantly increased odds of survival included witnessed arrest (pooled adjusted OR 2.46 [95\% Cl: $1.75-$ 3.45]), monitored arrest (pooled adjusted OR 1.85 [95\% Cl: 1.42-2.41]), arrest during daytime hours (pooled adjusted OR 1.35 [1.12-1.62]), and shockable rhythm (pooled adjusted OR 4.11 [95\% Cl: 3.01-5.59]). Intubation during IHCA was associated with decreased odds of survival (pooled adjusted OR 0.54 [95\% Cl: 0.42-0.70]).

Conclusions: Pre-arrest factors associated with reduced odds of survival to discharge include existing comorbidities (e.g. active malignancy, chronic kidney disease), and 
admission diagnosis of sepsis, while intra-arrest factors include unwitnessed arrest, unmonitored arrest, non-shockable rhythm, arrest outside of daytime hours, and intubation during arrest. Intubation during arrest was associated with reduced odds of survival. These results may inform discussions with patients when determining goalsof-care during admission, and consideration when determining cessation of IHCA management. 
Predicting Time to Death After the Withdrawal of Life Supporting Therapies Using Variability Analysis of Vital Signs Waveform Data

$\underline{\text { Scales, NB }}{ }^{1}$; Herry, $C^{1}$; van Beinum, $A^{3} ;$ Hogue, $M^{3}$; Hornby, $L^{2,3}$; Dhanani, $S^{3,4}$; Seely, $\mathrm{AJ}^{1,5,6}$

1 Ottawa Hospital Research Institute, Ottawa, Ontario, Canada

2 Canadian Blood Services, Ottawa, Ontario, Canada

3 Children's Hospital of Eastern Ontario Research Institute, Ottawa, Ontario, Canada

3 Children's Hospital of Eastern Ontario Research Institute, Ottawa, Ontario, Canada

4 Division of Pediatric Critical Care, Children's Hospital of Eastern Ontario, University

of Ottawa, Ottawa, Ontario, Canada

5 University of Ottawa, Ottawa, Ontario, Canada

6 Divisions of Thoracic Surgery \& Critical Care Medicine, Ottawa, Ontario, Canada

Background: Organ donation after circulatory determined death (DCD) accounts for $17 \%$ of all deceased organ donors in Canada, a fraction of its theoretical potential ${ }^{1}$. Up to half of all potential DCD patients are unable to donate because they do not die within currently required time limits to minimize organ ischemic damage ${ }^{2}$. The inability to predict time to death results in additional stress for families, a reduced number of successful donors, and increased costs for the health system. The DePPaRT (Death Physiology and Prediction After Removal of Therapy) study captured vital signs waveform data from 654 patients undergoing withdrawal of life sustaining therapy (WLST) in 22 centres in Canada, the Czech Republic, and the Netherlands. As reduced vital signs variability is associated with illness ${ }^{4,6,7}$, we hypothesized that the loss of heart rate and blood pressure variability in the hour prior to WLST might be useful in predicting time to death after WLST.

Objective: To develop a model based on vital signs waveform variability capable of predicting whether a patient will die within the timelines required for organ donation $(0.5,1$, and 2 hours after WLST).

Methods: Vital signs waveform data was available prior to WLST in 567 patients. This data was processed to obtain beat-to-beat event times series from the ECG and arterial blood pressure waveforms ( $R$ peak to $R$ peak interval $(R R I)$, systolic, diastolic, and pulse blood pressures), using CIMVA ${ }^{3,4}$. Each series was filtered to remove arrhythmias prior to the calculation of up to 53 measures of variability, using windows of 750 beats. We excluded patients that had prolonged WLST, namely patients with additional life supporting therapies removed after 10 minutes after the first act of WLST. Patients enrolled prior to and after March 2017 were used in the derivation (210 patients) and validation (105 patients) cohorts, respectively (Figure 1). All variability features were normalized to have a mean of 0 and a standard deviation of 1 . We used random survival forests ${ }^{9}$ in $\mathrm{R}$ to develop a predictive model. The model yielded a probability of dying within each 15-minute interval up until 24 hours after WLST for each patient (Figure 2a). Probabilities at specific times on these curves $(t=$ $0.5,1$, and 2 hours) were used as scores to predict if a given patient would die within these times. Features were ranked based on their importance over a 10 -fold cross validation of the training set. The model was retrained using only the highest ranked variables, adding one variable at a time. We used all 210 patients from the training set and the 15 features required for the AUC to plateau (Figure $2 b$ ) in a final model tested on the validation set.

Results: The derived model employing 15 variability features achieved a median AUC of $0.77,0.8$, and 0.77 for death within $0.5,1$, or 2 hours, respectively, on the validation set (Figure 3a). Nearly $91 \%$ of the patients with the highest scores died within 2 hours of WLST (Figure 3b). On our dataset, our model outperformed one of the highest performing models in the literature ${ }^{5,8}$ (Figure 3c).

Conclusion: For the first time, a predictive model using only heart rate and blood pressure variability data to predict time to death after WLST has been shown to 
achieve superior performance to models derived using clinical data ${ }^{5}$. In the future, we will combine and evaluate models incorporating both variability and additional clinical variables.

\section{Figure 1 - Patients Included in Prediction Model}

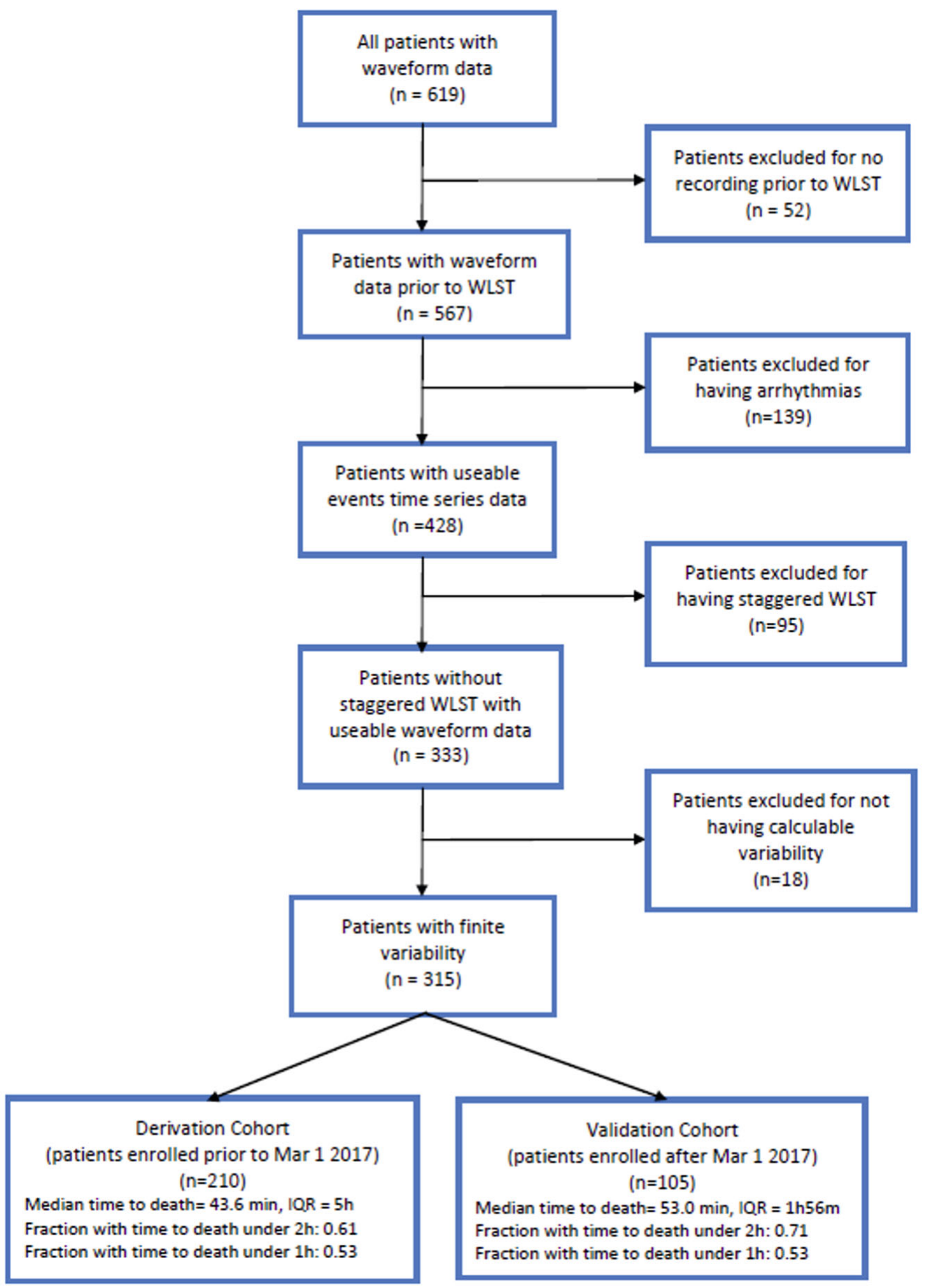


Figure 2
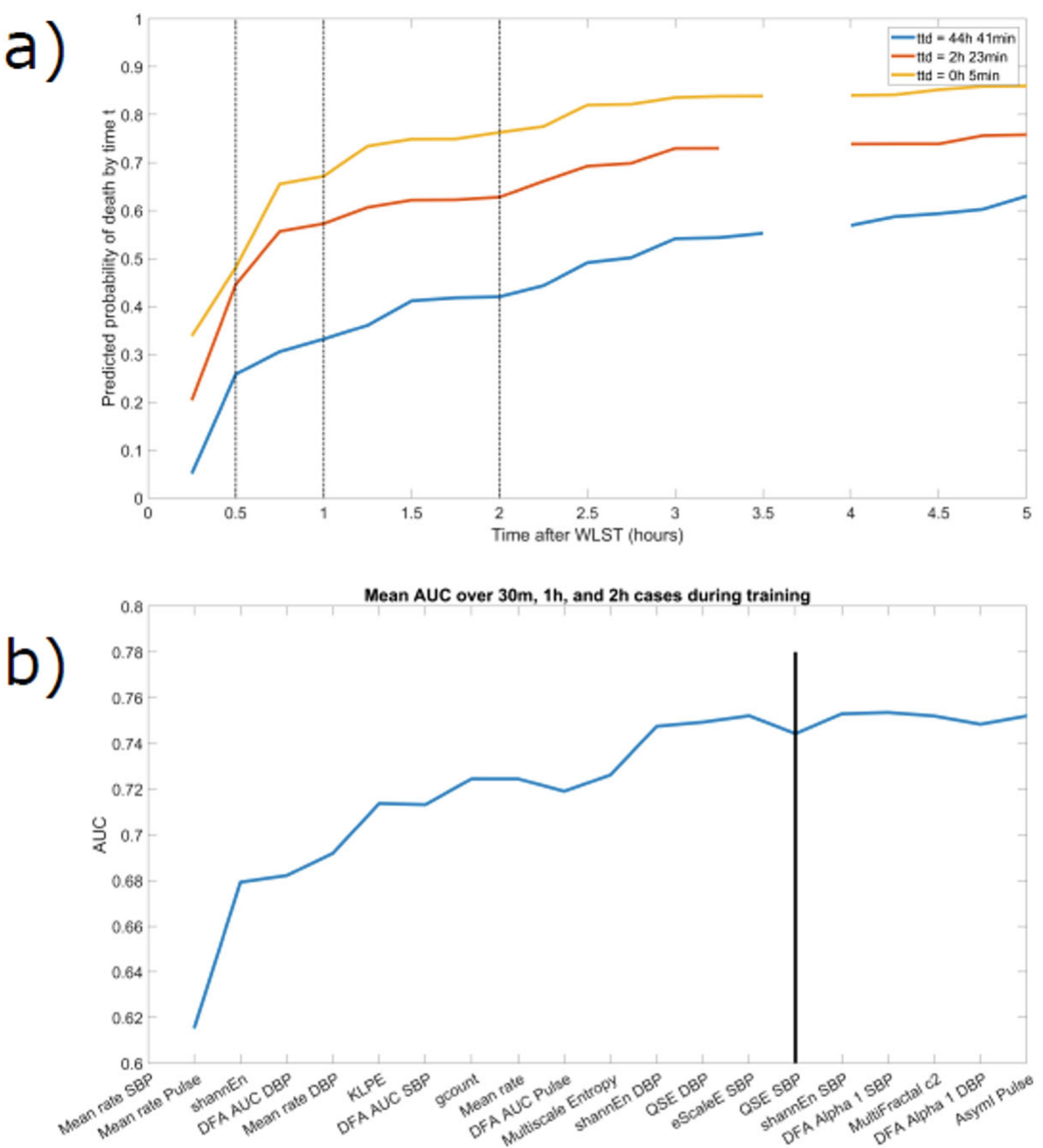

a) Example probability of dying curves generated by the model, for three patients with different time-to-death (ttd) values. The dotted black lines show the points used to generate the predictive score at the cutoffs of $0.5,1$, and 2 hours.

b) Average performance of the predictive model during training while adding the highest ranked features one at a time, starting from the second feature on the left. The black line shows the cutoff for features used in the final model.

shannEn = Shannon Entropy; DFA AUC = Detrended Fluctuation Analysis (Area under the curve); KLPE = Kullback-Leibler permutation entropy; gcount = Grid transformation feature: grid count; $\mathrm{QSE}=$ Quadtratic sample entropy; eScaleE = Embedding scaling exponent; AsymI $=$ Multiscale time irreversibility asymmetry index; MultiFractal c2 $=$ Multifractal spectrum cumulant of the second order; DFA Alpha $1=$ Detrended Fluctuation Analysis (alpha 1 scaling exponent); 
Figure 3

a)

\begin{tabular}{|c|c|c|c|}
\hline $\begin{array}{l}\text { Performance of } \\
\text { predictive model }\end{array}$ & $30 \mathrm{~m}$ & 1h & $2 \mathrm{~h}$ \\
\hline $\begin{array}{l}\text { AUC in Derivation } \\
\text { (IQR) }\end{array}$ & $\begin{array}{c}0.73 \\
(0.66,0.77)\end{array}$ & $\begin{array}{c}0.81 \\
(0.64,0.87)\end{array}$ & $\begin{array}{c}\mathbf{0 . 7 9} \\
(0.70,0.83)\end{array}$ \\
\hline AUC in Validation & 0.77 & 0.8 & 0.77 \\
\hline $\begin{array}{l}\text { Sensitivity in Derivation } \\
\text { (IQR) }\end{array}$ & $\begin{array}{c}0.88 \\
(0.78,0.89)\end{array}$ & $\begin{array}{c}\mathbf{0 . 8 3} \\
(0.75,0.91)\end{array}$ & $\begin{array}{c}\mathbf{0 . 7 9} \\
(0.77,0.92)\end{array}$ \\
\hline Sensitivity in Validation & 0.69 & 0.73 & 0.79 \\
\hline $\begin{array}{l}\text { Specificity in Derivation } \\
\text { (IQR) }\end{array}$ & $\begin{array}{c}0.67 \\
(0.58,0.77)\end{array}$ & $\begin{array}{c}0.7 \\
(0.52,0.87)\end{array}$ & $\begin{array}{c}0.77 \\
(0.7,0.88)\end{array}$ \\
\hline Specificity in Validation & 0.81 & 0.80 & 0.77 \\
\hline
\end{tabular}

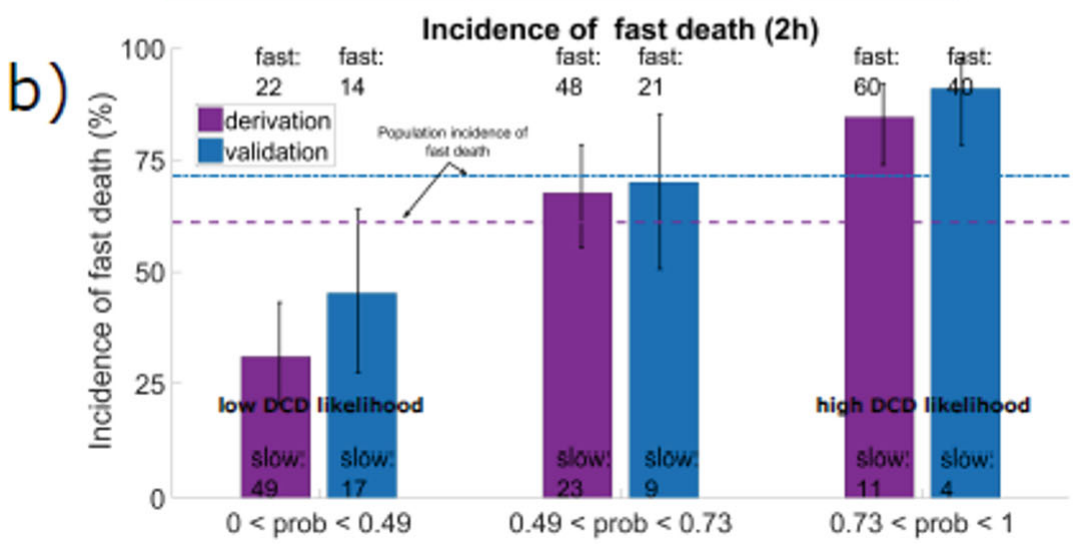

C)

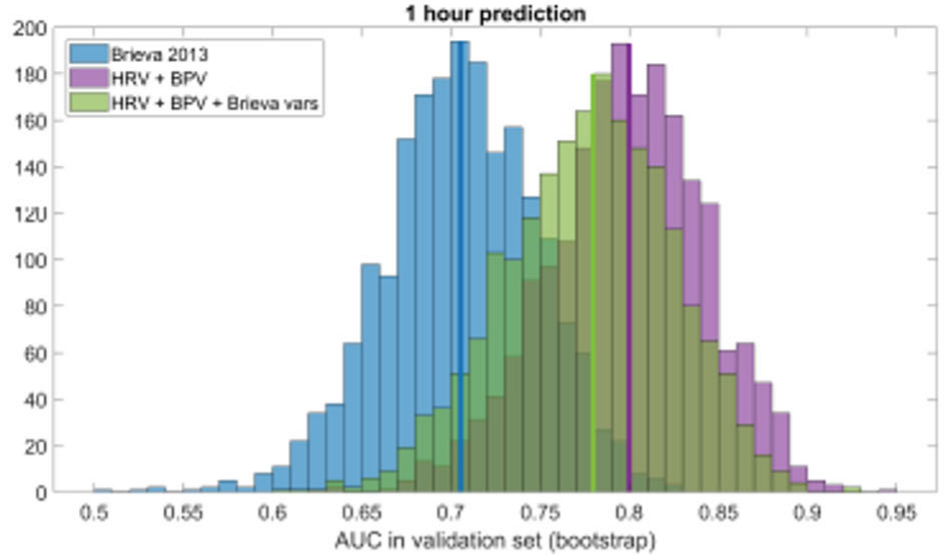

a) Table showing the AUC, sensitivity, and specificity results for the derivation and validation cohorts for the $30 \mathrm{~m}, 1 \mathrm{~h}$, and $2 \mathrm{~h}$ prediction models.

b) The model scores for the derivation (purple) and validation (blue) sets were divided into low, medium, and high likelihood groups for DCD for the 2 hour prediction model. The incidence of fast death was plotted for each risk group.

c) Histograms of the AUC after 2000 bootstrap iterations on the validation set, for Brieva's 2013 model [8], the variability only model (HRV+BPV), and a random survival forest model including variability as well as the variables used in the Brieva model. The solid lines indicate the median AUC. The Brieva model employed 6 clinical features (respiratory rate, Glasgow coma scale, positive end-expiratory pressure, systolic blood pressure, $\mathrm{pH}$, analgesia) as well as the the clinician's prediction of time to death. 


\section{References}

1. "Deceased Organ Donor Potential in Canada", report for the Canadian Institute for Health Information, December 2014

(https://secure.cihi.ca/free products/OrganDonorReport ENweb.pdf)

2. Hodgson, Russell, Alastair L. Young, Magdy A. Attia, and J. Peter A. Lodge. "Impact of a National Controlled Donation After Circulatory Death (DCD)

Programme on Organ Donation in the UK: A 10 Year Study." American Journal of Transplantation.

3. Dynamical Analysis Laboratory, "CIMVA Exectuable Version 4.3 User Guide" (2017). https://www.ohridal.org/cimva/CIMVA UserGuide.pdf

4. Bravi, Andrea, André Longtin, and Andrew JE Seely. "Review and classification of variability analysis techniques with clinical applications." Biomedical engineering online 10, no. 1 (2011): 90.

5. Munshi, Laveena, Sonny Dhanani, Sam D. Shemie, Laura Hornby, Genevieve Gore, and Jason Shahin. "Predicting time to death after withdrawal of lifesustaining therapy." Intensive care medicine 41, no. 6 (2015): 1014-1028.

6. Goldberger, Ary L. "Non-linear dynamics for clinicians: chaos theory, fractals, and complexity at the bedside." The Lancet 347, no. 9011 (1996): 1312-1314.

7. Seely, Andrew JE, and Peter T. Macklem. "Complex systems and the technology of variability analysis." Critical care 8, no. 6 (2004): R367.

8. Brieva J, Coleman N, Lacey J, Harrigan P, Lewin TJ, Carter GL. Prediction of death in less than 60 minutes following withdrawal of cardiorespiratory support in ICUs. Critical care medicine. 2013 Dec 1;41(12):2677-879.

9. Ishwaran H, Kogalur UB, Blackstone EH, Lauer MS. Random survival forests. The annals of applied statistics. 2008;2(3):841-60. 
Predictors of Graft Loss Following Kidney Transplantation: Systematic Review and Meta-Analysis

Foroutan, Farid ${ }^{1}$,2. Clark, Kathryn ${ }^{2}$; Friesen, Erik ${ }^{1}$; Lee, Yung ${ }^{1}$, Akhtar, Aemal $^{1}$; Arseneau, Erika ${ }^{1}$; Ribic, Christine ${ }^{1}$; Treleavan, Darin ${ }^{1}$; Guyatt, Gordon H. ${ }^{1}$; Meade, Maureen O. ${ }^{1}$

1 Department of Health Research Methods, Evidence, and Impact, McMaster, Hamilton, Ontario, Canada

2 Multi-Organ Transplant, University Health Network, Toronto, Ontario, Canada

Introduction: Selection of recipients and donors for kidney transplantation is informed by factors that influence the longevity of the graft. Previous literature identifying predictors and risk scores for graft loss are developed through either single center studies or registries. Investigators have not thus far conducted a systematic review of these studies.

Objective: Our systematic review and meta-analysis aims to identify all predictors and provide best estimates of their association with 1-year death censored graft loss in adult primary kidney transplant recipients.

Methods: We systematically searched MEDLINE, EMBASE, Cochrane database of systematic reviews, Cochrane central registries, and PubMed supplemental for eligible primary studies. We meta-analyzed the independent association (identified with multivariable regression models) between any risk factor (related to donor, recipient, and the transplant operation), and 1-year death censored graft loss. Our review utilized Grading of Recommendations, Assessment, Development, and Evaluation for assessing the quality of the evidence. We pooled effect estimates using the random effects or fixed effects framework, where appropriate. Studies defined extended criteria donors as $>60$ years of age or age 50 to 59 years with two of three associated risk factors-history of cerebrovascular accident, hypertension, or serum creatinine greater than $1.5 \mathrm{mg} / \mathrm{dL}$ and delayed graft function as the need for dialysis within the first week post-transplant.

Results: High quality evidence identified older recipient age (HR 1.17 per 10-year increase, $95 \% \mathrm{Cl} 1.09$ to 1.25 ), older donor age (HR 1.11 per 10 -year increase, $95 \% \mathrm{Cl}$ 1.04 to 1.18), extended criteria donors (HR 1.35, 95\% Cl 1.28 to 1.42), living donor (HR $0.65,95 \% \mathrm{Cl} 0.55$ to 0.76 ), increasing number of human leukocyte antigen (HLA) mismatches (HR 1.08 per 1 mismatch increase, 95\% Cl 1.07 to 1.09), and delayed graft function (HR 1.89, 95\% Cl 1.46 to 2.47 ) as predictors for 1-year graft loss (Table 1). High quality evidence also excluded associations of cold ischemic time, recipient race, recipient $\mathrm{BMI}$, and recipient diabetes with 1-year graft loss.

Conclusion: Donor type and quality, delayed graft function, recipient age, donor age, and HLA mismatch all appear to predict 1-year graft survival. The impact of each factor is, however, modest, suggesting the need for a predictive model that would incorporate multiple factors. The results of this study may be useful to inform future guidelines and predictive models. 
Prevalence and Prognosis of Critically III Patients with a Failed Cuff Leak Test: A Retrospective Observational Study

Piticaru, Joshua ${ }^{1}$; Lewis, Kim¹; Aucoin, Philip²; McConnell, Meghan²,3 Jaeschke, Roman $^{1,4}$; Almenawer, Saleh ${ }^{1}$; Alhazzani, Waleed ${ }^{1,4}$

1 Department of Medicine, McMaster University, Hamilton, Ontario, Canada

2 Department of Innovation in Medical Education, University of Ottawa, Ottawa, Ontario, Canada

3 Department of Anesthesiology and Pain Medicine, University of Ottawa, Ottawa, Ontario, Canada

4 Department of Health Research Methods, Evidence, and Impact, McMaster University, Hamilton, Ontario, Canada

Introduction: Post-extubation stridor (PES) is a well-known complication of intubation resulting from laryngeal edema. The cuff leak test $(C L T)$ is frequently used to predict PES, although demonstrates limited diagnostic performance and may expose patients to unnecessary avoidance of extubation.

Objectives: This study was conducted to 1) compare the incidence of a failed CLT in critically ill patients that are either at a high or average risk of PES; 2 ) identify risk factors for laryngeal edema in the average ICU patient; and 3) compare characteristics of patients with a failed CLT compared with age and sex-matched controls with a cuff leak.

Methods: A single-centered case-control study of mechanically ventilated, critically ill patients was conducted from September 2012 to August 2017. Controls were matched for age and sex. Binomial logistic regression was performed to assess the significance of variables associated with a failed CLT.

Results: We identified 961 documented cuff leak tests performed in 3805 patients $(25.5 \%)$ over a five-year period, of which 88 were failed $(9.2 \%)$. Most patients with a failed CLT were not clearly high risk for PES based on historical features (53/88, $60.2 \%$ ). However, patients deemed at high risk of PES were more likely to have a failed CLT relative to patients with average risk of PES (OR 4.10 (95\% CI 1.84-9.17, p $=0.001$ ), have longer duration from CLT to extubation ( 2.3 vs 1.1 days, $p=0.03$ ), and increased rate of tracheostomy $(9.1 \%$ vs $0 \%, p=0.004)$. Failed CLT was not associated with intubation indication, endotracheal tube size, fluid balance, increased rates of PES or reintubation.

Conclusions: In a general ICU, most patients with a failed CLT are not identifiably at high risk for PES. The presence of a failed CLT is associated with delayed extubation and increased tracheostomy rates. These results inform the upcoming COMIC study, a randomized control trial investigating whether use of a CLT impacts relevant clinical outcomes in average risk patients.

\section{References}

1. Lewis K, Alhazzani W. 2017. The cuff leak test prior to extubation: A practice based on limited evidence. Saudi Critical Care Journal, 1(6):22-24. 
Prognostic Accuracy of the Hamilton Early Warning Score (HEWS) Among Hospitalized Patients Assessed by a Rapid Response Team

Fernando, Shannon M. ${ }^{1,2}$; Fox-Robichaud, Alison E. ${ }^{3,4}$; Rochwerg, Bram ${ }^{3,4}$; Cardinal, Pierre $^{1}$; Seely, Andrew J. E. ${ }^{1,5,6}$; Perry, Jeffrey J. ${ }^{2,5,6}$; Mclsaac, Daniel I. ${ }^{5,6,7}$; Skitch, Steven ${ }^{3,8}$; Tam, Benjamin ${ }^{3}$; Hickey, Michael ${ }^{1,2,5}$, Reardon, Peter M. ${ }^{1,2}$; Tanuseputro, Peter $^{5,6,8}$; Kyeremanteng, Kwadwo ${ }^{1,6,8,9}$

1 Division of Critical Care, Department of Medicine, University of Ottawa, Ottawa, Ontario, Canada

2 Department of Emergency Medicine, University of Ottawa, Ottawa, Ontario, Canada

3 Department of Medicine, Division of Critical Care, McMaster University, Hamilton, Ontario, Canada

4 Department of Health Research Methods, Evidence, and Impact, McMaster

University, Hamilton, Ontario, Canada

5 School of Epidemiology and Public Health, University of Ottawa, Ottawa, Ontario,

Canada

6 Clinical Epidemiology Program, Ottawa Hospital Research Institute, The Ottawa

Hospital, Ottawa, Ontario, Canada

7 Department of Anesthesiology and Pain Medicine, University of Ottawa, Ottawa, Ontario, Canada

8 Division of Emergency Medicine, Department of Medicine, McMaster University, Hamilton, Ontario, Canada

9 Division of Palliative Care, Department of Medicine, University of Ottawa, Ottawa, Ontario, Canada

Introduction: Patients admitted to hospital wards are at risk of future deterioration. Rapid Response Teams (RRTs) respond to hospitalized patients with deterioration, and help determine subsequent management, including Intensive Care Unit (ICU) admission. In order to optimize disposition, RRTs must appropriately identify patients at risk of adverse clinical outcomes.

Objectives: We sought to evaluate the prognostic accuracy of the Hamilton Early Warning Score (HEWS) for prediction of in-hospital mortality and ICU admission among hospitalized patients. The prognostic accuracy of HEWS was compared to the National Early Warning Score 2 (NEWS2). We secondarily evaluated the accuracy of HEWS in a subgroup of RRT patients with suspected infection.

Methods: We utilized prospectively collected registry data (2012-2016) from two hospitals in Ottawa, Ontario. Consecutive hospitalized adult patients assessed by the RRT were included. Vital signs and laboratory values were collected at the time of RRT assessment. Patients were followed to the point of in-hospital death or hospital discharge. 'Suspected infection' was defined as concomitant administration of antibiotics and sampling of body fluid cultures. The Number Needed to Examine (NNE) was calculated, which indicates the number of patients that need to be evaluated in order to detect one patient who will die in-hospital, and is an indirect measure of the cost-effectiveness of each alert.

Results: 5,995 patients were included in analysis, of whom 1,833 (30.6\%) died inhospital. 1,708 patients (28.5\%) met criteria for 'suspected infection'. A HEWS above the low-risk threshold $(\geq 3)$ had a sensitivity of $90.8 \%(95 \% \mathrm{Cl}: 89.4-92.1)$ and specificity of $32.4 \%$ (95\% Cl: 31.0-33.9) for in-hospital mortality, with a NNE of 2.7 (95\% Cl: 2.6-2.8). A HEWS above the moderate-risk threshold $(\geq 5)$ had a sensitivity of $73.4 \%(95 \% \mathrm{Cl}: 71.3-75.4)$ and specificity of $60.7 \%$ (95\% Cl: $59.2-62.2)$ for in-hospital mortality, with a NNE of $2.2(95 \% \mathrm{Cl}: 2.1-2.3)$. Finally, a high-risk HEWS ( $\geq 9)$ had a sensitivity of $33.9 \%$ (95\% Cl: $31.8-36.2)$ and specificity of $99.5 \%(95 \% \mathrm{Cl}: 99.3-99.7)$ for in-hospital mortality, with a NNE of 1.0 (95\% Cl: 1.0-1.1). The area under the receiver operating characteristic $(\mathrm{ROC})$ curve for mortality was $0.743(95 \% \mathrm{Cl}$ : $0.730-$ $0.757)$ for HEWS, and 0.754 (95\% Cl: 0.733-0.782) for NEWS2. Among patients with 
suspected infection, a HEWS $\geq 3$ had a sensitivity of $91.2 \%$ (88.6-93.4) and specificity of 33.3 (95\% Cl 30.6-36.1) for in-hospital mortality, with a NNE of 2.5 (95\% Cl: 2.4-2.5). A HEWS $\geq 5$ had a sensitivity of 74.8 (95\% Cl: 70.8-78.4) and specificity of 54.7 (51.8$57.5)$, with a NNE of $2.4(95 \% \mathrm{Cl}: 2.3-2.5)$.

Conclusions: The HEWS has comparable clinical accuracy to NEWS2 for prediction of in-hospital mortality among RRT patients. This external application of the HEWS suggests that it may be a valuable tool in the risk-stratification of hospitalized patients with acute deterioration. Early detection, appropriate risk-stratification, and optimal disposition in this patient population have been associated with improved outcomes. 
Prognostic Accuracy of the HEART Score for Prediction of Major Adverse Cardiac Events in Patients Presenting with Chest Pain - A Systematic Review and Meta-analysis

Fernando, Shannon M. ${ }^{1,2}$; Tran, Alexandre ${ }^{3,4}$; Cheng, Wei ${ }^{5}$; Rochwerg, Bram ${ }^{6,7}$; Taljaard, Monica ${ }^{3,5}$; Thiruganasambandamoorthy, Venkatesh ${ }^{1,3,5}$; Kyeremanteng, Kwadwo ${ }^{2,5}$; Perry, Jeffrey J. ${ }^{1,3,5}$

1 Department of Emergency Medicine, University of Ottawa, Ottawa, Ontario, Canada

2 Division of Critical Care, Department of Medicine, University of Ottawa, Ottawa, Ontario, Canada

3 School of Epidemiology and Public Health, University of Ottawa, Ottawa, Ontario, Canada

4 Department of Surgery, University of Ottawa, Ottawa, Ontario, Canada

5 Clinical Epidemiology Program, Ottawa Hospital Research Institute, Ottawa, Ontario, Canada

6 Department of Medicine, Division of Critical Care, McMaster University, Hamilton, Ontario, Canada

7 Department of Health Research Methods, Evidence, and Impact, McMaster University, Hamilton, Ontario, Canada

Introduction: Risk-stratification of patients presenting with chest pain is crucial for identifying potentially life-threatening outcomes. The HEART (History, Electrocardiogram, Age, Risk factors, Troponin) score has been proposed for prediction of major adverse cardiac events (MACE, a composite outcome including mortality, myocardial infarction, or coronary revascularization) in this population. Objectives: We sought to summarize all studies assessing the prognostic accuracy of the HEART score for prediction of MACE in adult patients presenting with chest pain. Where available, accuracy of the thrombolysis in myocardial infarction (TIMI) score was determined.

Methods: We searched six databases (including Medline, EMBASE, and Web of Science) from inception through January 22, 2018. We included English-language studies using the HEART score for the prediction of short-term MACE in adult patients presenting with chest pain. We followed PRISMA guidelines and the Cochrane Handbook for Systematic Reviews of Diagnostic Test Accuracy. Two reviewers independently extracted data and assessed study quality using the Quality Assessment of Diagnostic Accuracy Studies (QUADAS-2) tool. The main outcome was short-term (i.e. 30-day or 6-week) incidence of MACE.

Results: We included 29 studies $(n=42,650)$ in pooled analysis. A HEART score above the low-risk threshold $(\geq 4)$ had a sensitivity of $95.6 \%$ (95\% confidence interval [CI]: 92.8-97.3) and specificity of $44.0 \%(95 \% \mathrm{Cl}: 38.1-50.0)$ for MACE, while a TIMI score above the low-risk threshold $(\geq 2)$ had a sensitivity of $87.6 \%(95 \% \mathrm{Cl}: 78.7-93.1)$ and specificity of $46.0 \%(95 \% \mathrm{Cl}$ : $36.7-55.6)$. A high-risk HEART score $(\geq 7)$ had a sensitivity of $39.5 \%(95 \% \mathrm{Cl}: 31.6-48.1)$ and specificity of $95.0 \%$ (95\% Cl: $92.2-96.6)$ for MACE, while a high-risk TIMI score $(\geq 6)$ was $2.8 \%$ sensitive $(95 \% \mathrm{Cl}: 0.8-9.6)$, but 99.6\% (95\% Cl: 98.5-99.9) specific for MACE. The pooled area under the receiver operating characteristic curve for prediction of MACE was 0.81 (95\% Cl: $0.76-0.86)$ for the HEART score, and 0.73 (95\% Cl: 0.68-0.79) for TIMI.

Conclusions: The HEART score has excellent performance for prediction of MACE in chest pain patients. Clinicians should preferentially utilize the HEART score for riskstratification of this patient population. 
Promoting Professional Development of Critical Care Research Coordinators through Annual Workshops

O'Hearn, Katie ${ }^{1,2}$; Ward, Roxane ${ }^{2,3}$; Foster, Denise ${ }^{2,4}$; Clayton, Lucy L $^{2,5}$; Julien, Lisa $^{2,6}$; Marten, Nicole ${ }^{2,7}$; Matte, Andrea ${ }^{2}$, McDonald, Ellen ${ }^{2,8,10}$; Smith, Orla ${ }^{2,9}$; Van Huyse, Judy ${ }^{2,10}$; Zytaruk, Nicole ${ }^{2,11,12}$; Cook, Deborah ${ }^{11,12}$

1 Children's Hospital of Eastern Ontario, Ottawa, Ontario, Canada

2 Canadian Critical Care Research Coordinators Group, Canada

3 Ottawa Hospital Research Institute, Ottawa, Ontario, Canada

4 Vancouver General Hospital, Vancouver, British Columbia, Canada

5 St. Justine Hospital, Montréal, Quebec, Canada

6 Queen Elizabeth II HSC, Halifax, Nova Scotia, Canada

7 St. Boniface Hospital, Winnipeg, Manitoba, Canada

8 Hamilton General Hospital, Hamilton, Ontario, Canada

9 St. Michael's Hospital, Toronto, Ontario, Canada

10 Toronto Sick Children's Hospital, Toronto, Ontario, Canada

11 McMaster University, Hamilton, Ontario, Canada

12 St. Joseph's Healthcare Hamilton, Hamilton, Ontario, Canada

Background: Research coordinators (RC) are essential to critical care research yet often work in isolation from peers and have limited opportunities for professional growth and development. In 2004, the Canadian Critical Care Trials Group (CCCTG) formed the Canadian Critical Care Research Coordinators Group (CCCRCG) to provide a forum for dedicated education and an opportunity for networking.

Objective: The objective of this study is to describe faculty, content, and evaluations from a series of workshops provided to Canadian RCs by the CCCRCG.

Methods: We reviewed annual workshop agendas for speakers and content as well as responses on evaluation forms. Speakers were categorized as: RC, CCCTG Investigators $(\mathrm{Cl})$ or Invited Experts (IE). Workshop content was categorized as: (1) Professional Growth and Development (PG\&D): presentation content aiming to improve the competence, skill, and effectiveness of RCs; (2) Regulatory Requirements $(\mathrm{RR})$ : presentation content related to clinical research regulations; and (3) Education (ED): presentation content related to diagnosis, treatment and therapeutic modalities in critical care or general information about critical care research. Evaluation forms used a 5-point Likert scale (1=unacceptable; $5=$ excellent) to rate speakers. Workshop attendees were invited to provide comments regarding current and future content. Two authors synthesized the narrative comments by theme.

Results: On average, 40 RCs attended each of the 14 workshops (2004-2017). Of the 96 sessions presented, 37 (39\%) were related to PG\&D, 35 (36\%) ED, and $24(25 \%)$ RR. Amongst the 122 speakers, the majority were RCs $81(66 \%)$, with $\mathrm{Cl}$ and IE representing $21(17 \%)$ and $14(11 \%)$ of speakers respectively. Speakers were rated 2008 times. The average rating was 4.4 (SD 0.74 ) (very good) on a $1-5$ scale; $52 \%$ of ratings were 5 (excellent). Using a Jonckheere-Terpstra test, it was determined that evaluation ratings are improving over time $(p=0.01)$. Workshop attendee comments provided positive feedback on the diversity of topics and speakers. RCs reported networking opportunities, interactive discussions and learning from more experienced $\mathrm{RCs}$ as the most valued aspects of the workshops. Areas for improvement included more time for group discussion and interactive sessions. Suggestions for future workshop content focused on practical day-to-day activities such as grant writing, budget preparation, consent and co-enrolment, regulatory requirements, time management, research methodology and increasing productivity.

Conclusions: The Canadian Critical Care Research Coordinators have broad learning needs. Professional growth and development is highly valued. Attendees at national Workshops appreciate increasing their knowledge base, networking with colleagues, and interactive discussions. RCs desire education related to practical aspects of their 
work, including improving efficiency and productivity while adhering to regulatory requirements. Workshop evaluations have improved over the 14 years of operation.

Figure 1

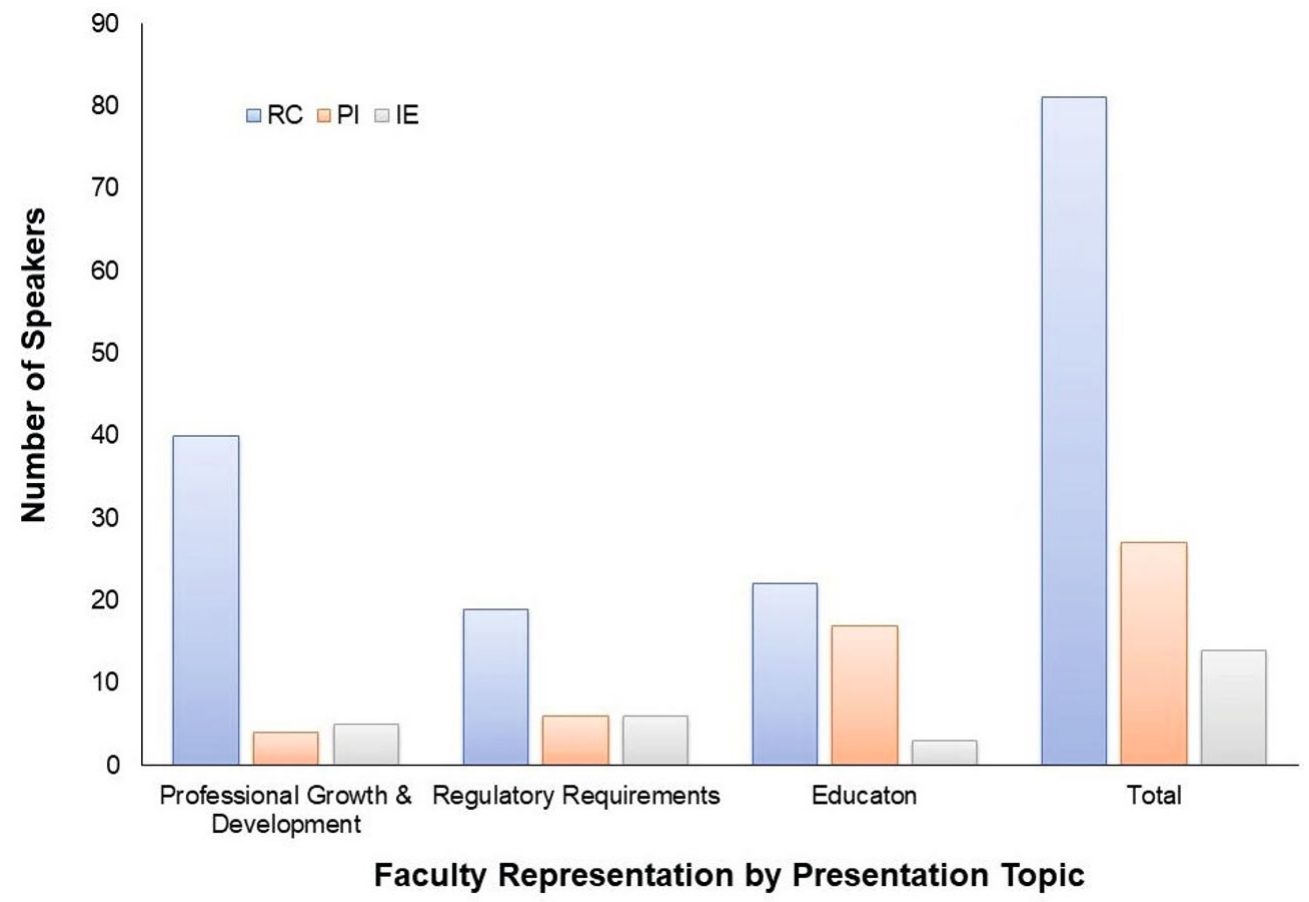

Figure 2

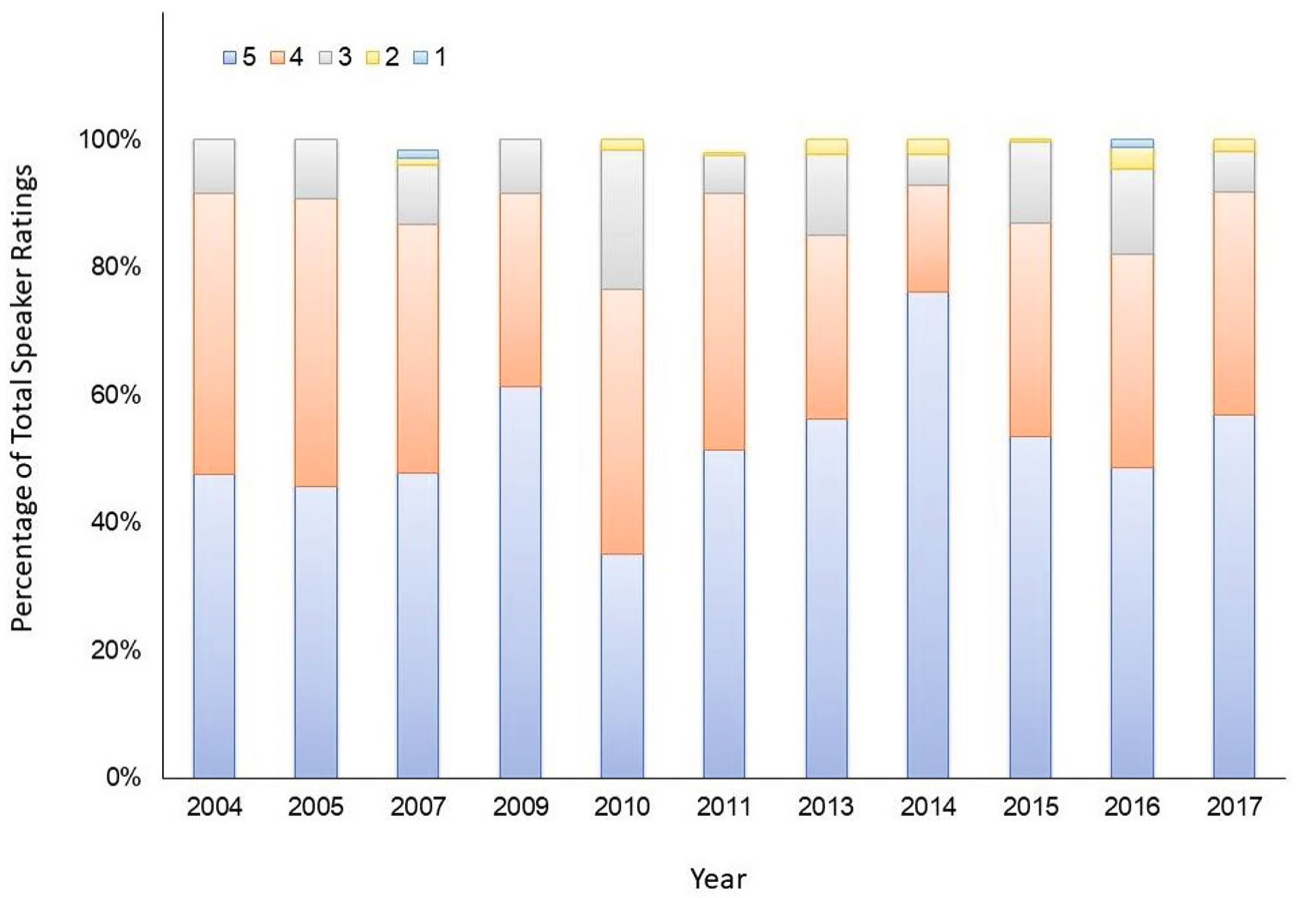




\section{Quantifying the Academic Activities of Canadian Critical Care Research} Coordinators Group (CCCRCG)

O'Hearn, Katie ${ }^{1,2}$; Ward, Roxane ${ }^{2,3}$; Foster, Denise ${ }^{2,4}$; Clayton, Lucy 2,5 ; Julien, Lisa $^{2,6}$; Marten, Nicole ${ }^{2,7}$; Matte, Andrea ${ }^{2}$, McDonald, Ellen²,8,10; Smith, Orla ${ }^{2,9}$; Van Huyse, Judy ${ }^{2,10}$; Zytaruk, Nicole ${ }^{2,11,12}$; Cook, Deborah ${ }^{11,12}$

1 Children's Hospital of Eastern Ontario, Ottawa, Ontario, Canada

2 Canadian Critical Care Research Coordinators Group, Canada

3 Ottawa Hospital Research Institute, Ottawa, Ontario, Canada

4 Vancouver General Hospital, Vancouver, British Columbia, Canada

5 St. Justine Hospital, Montréal, Quebec, Canada

6 Queen Elizabeth II HSC, Halifax, Nova Scotia, Canada

7 St Boniface Hospital, Winnipeg, Manitoba, Canada

8 Hamilton General Hospital, Hamilton, Ontario, Canada

9 St Michael's Hospital, Toronto, Canada

10 Toronto Sick Children's Hospital, Toronto, Ontario, Canada

11 McMaster University, Hamilton, Ontario, Canada

12 St. Joseph's Healthcare Hamilton, Hamilton, Ontario, Canada

Background: Critical Care Research Coordinators (RCs) affiliated with the Canadian Critical Care Trial Group (CCCTG) collaborate with Investigators both in Canada and internationally. To-date, the academic activities of Research Coordinators (RC) have not been well described. After inception of the CCCRCG in 2004, five important initiatives have supported the professional development of the members: 1) Annual Workshops starting in 2004; 2) Research Grant competition to fund RC-led projects launched in 2011; 3) Travel grant competition instituted in 2015 to support travel to CCCTG meetings and CCCRCG Workshops; 4) a National Platform Research Coordinator (0.5 FTE) was appointed to elicit and support RC learning needs; and 5) a Webinar series was launched in 2016.

Objective: The objective of this project is to describe the academic activities of Canadian Critical Care Research Coordinators over the past 22 years.

Methods: We developed a Curriculum Vitae (CV) to document academic activities of CCCRCG between1996-2017. Abstracts, manuscripts, and grants which included RCs as either (co-)authors or (co-)investigators were included. Duplicate citations and citations referencing activities not related to critical care or to CCCTG-work were verified with the authors and removed. We documented the number of citations and grants with RCs as first or last author to highlight RC leadership of research initiatives. We checked references, and in duplicate, counted the number of citations. We calculated the total funding amount of RC-led grants and grants for which RCs were contributors. Grant contributions were checked by 2 RCs.

Results: The CCCRCG CV includes 426 independent entries: 258 abstracts, 139 manuscripts, and 29 grants. A RC is listed as first or last author for $133(31 \%)$ independent entries, including $96(37 \%)$ abstracts and $22(16 \%)$ manuscripts. A Spearman's rank-order correlation was run to assess the relationship between time in years and total number of manuscripts published. Preliminary analysis showed the relationship to be monotonic, as assessed by visual inspection of a scatterplot. There was a strong positive correlation between time (in years from 1996 to 2017) and total number of manuscript inclusions, $\mathrm{rs}(22)=.887, \mathrm{p}<.001$. Similarly, there was a strong positive correlation between time and total number of manuscripts inclusions with an $\mathrm{RC}$ as first or last author, $\mathrm{rs}(22)=.639, \mathrm{p}=.002$. CCCRCG members received funding for 15 grants for which the Principal Investigator $(\mathrm{PI})$ was a RC, with a total budget amount of $\$ 47,000$. Additionally, RCs have been involved as collaborators in another 14 grants, with a total budget of $\$ 3,222,636$. Overall, RCs have supported the grant process for studies with a total budget of $\$ 3,269,636$. Prior to the introduction of the RC Grant competition in 2011, the number of grants for which a RC was the PI was $1(8 \%)$ 
whereas in 2011 or later, a RC was the PI on 14 (82\%) grants.

Conclusions: As key members of CCCTG, RCs have led and collaborated on numerous abstracts, manuscripts, and grants. Both the annual volume of activity and the level of "contributorship" has increased over time. Grants with RCs as PIs have increased substantially since the introduction of the RC Grant competition in 2011. The productivity of CCCRCG has been supported by nationally funded initiatives from the CCCTG and we anticipate that this academic growth will be sustained in years to come.

Figure 1

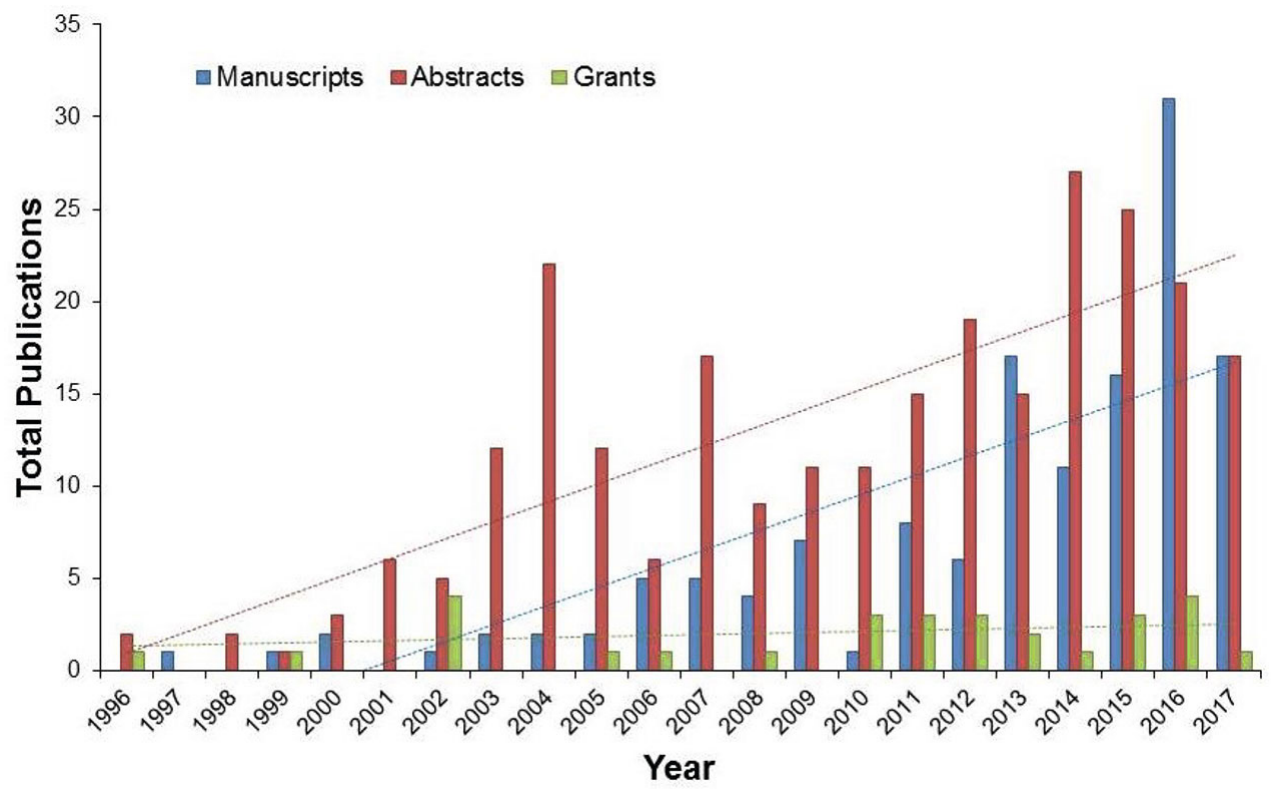

Figure 2

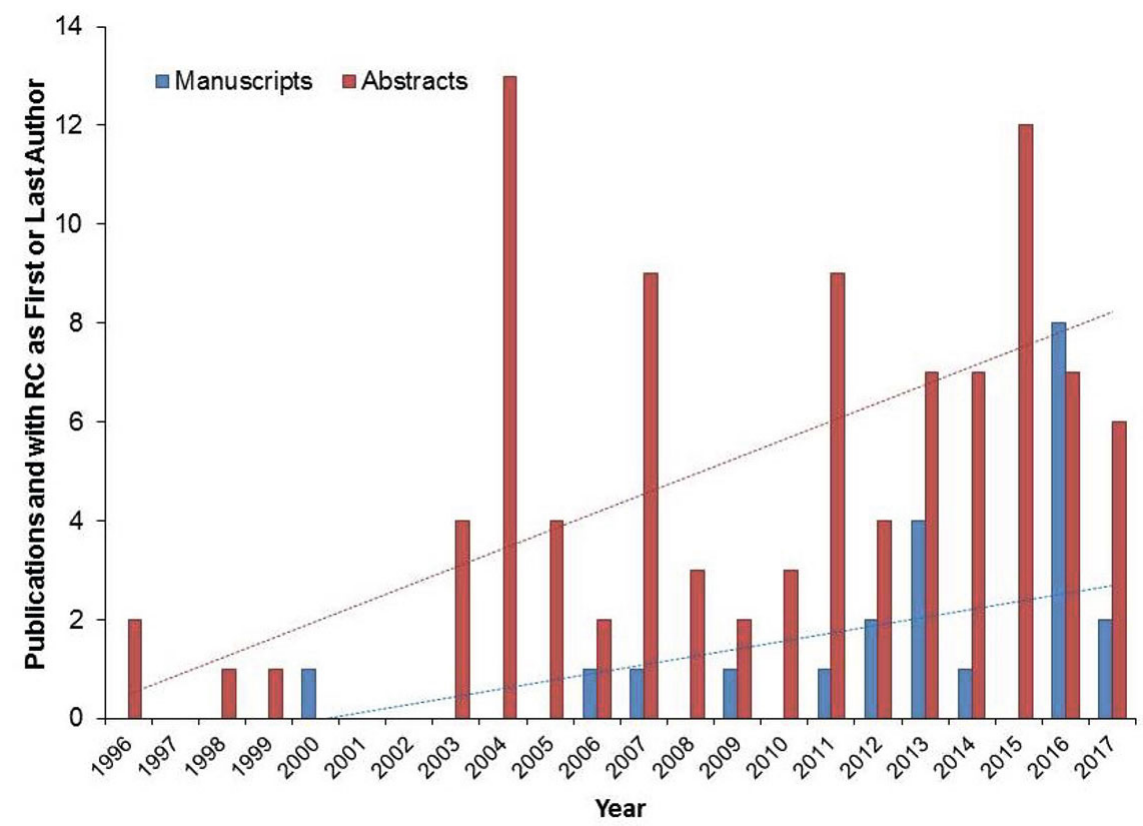


Figure 3

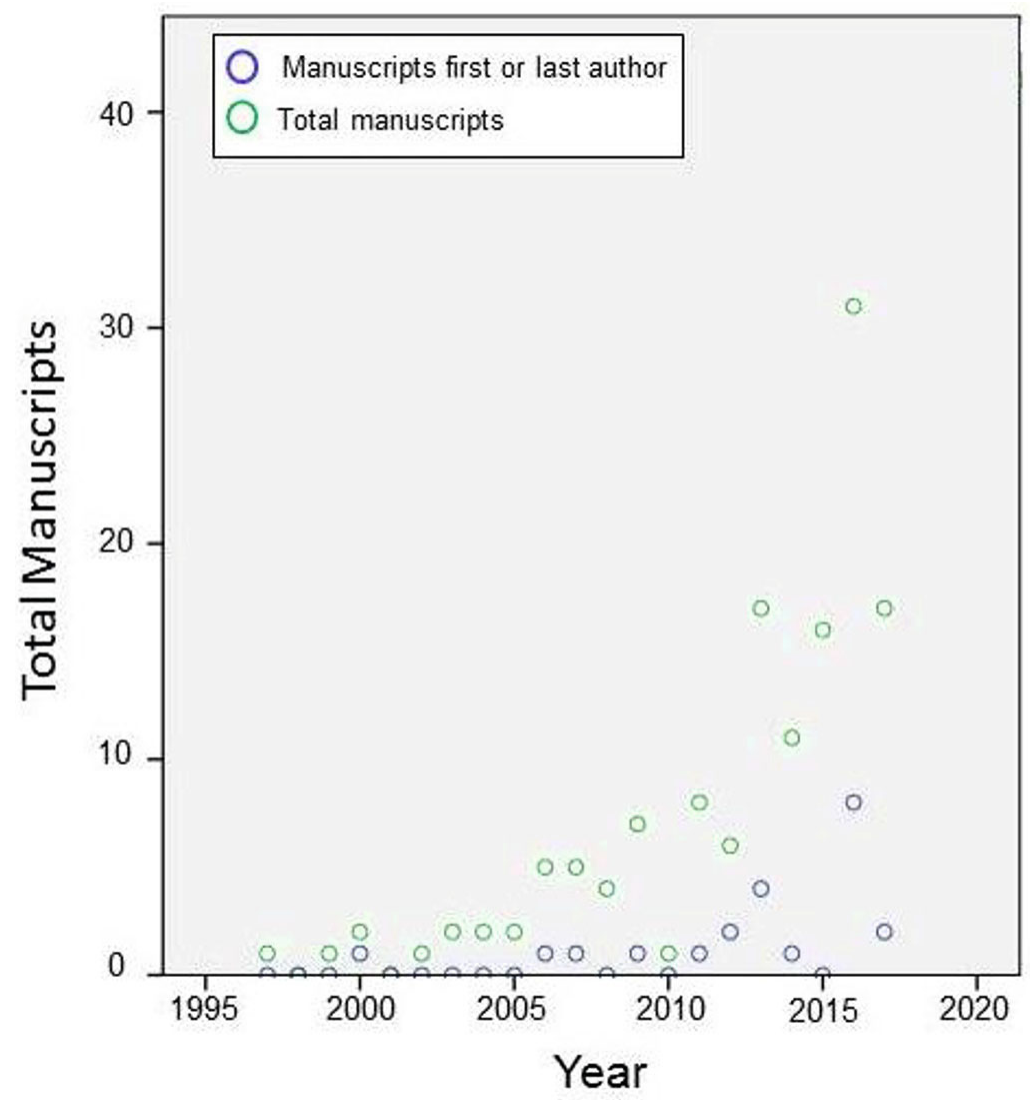


Survey of Canadian Intensive Care Physicians' Attitudes Towards a Presumed Consent Model for Deceased Organ Donation

Weiss, Matthew J. ${ }^{1,2,3}$; English, Shane W.4,5; Mohr, Jim³; Lauzier, François ${ }^{2,6,7}$; Turgeon, Alexis ${ }^{2,6}$; Dhanani, Sonny ${ }^{8}$; Shemie, Sam D. 3,9 ; Knoll, Gregory ${ }^{4,5}$; Fergusson, Dean ${ }^{4,5}$; D’Aragon, Frederick ${ }^{10}$; Anthony, Samantha J. ${ }^{11}$; McIntyre, Lauralyn ${ }^{4,5}$; HajMoustafa, Adnan ${ }^{12}$; Hartell, David ${ }^{13}$; Chassé, Michaël ${ }^{12,14}$

1 Department of Pediatrics, Université Laval, Faculté de Médecine, Quebec; Transplant Québec, Montréal, Québec City, Québec, Canada 2 CHU de Québec - Université Laval Research Center, Population Health and Optimal Health Practices Research Unit, Traumatology-Emergency-Critical Care Medicine, Université Laval, Québec City, Québec, Canada

3 Donation and Transplantation, Canadian Blood Services, Ottawa, Ontario, Canada 4 Department of Medicine, University of Ottawa, Ottawa, Ontario, Canada

5 Clinical Epidemiology Program, Ottawa Hospital Research Institute, Ottawa, Ontario, Canada

6 Division of Critical Care Medicine, Department of Anesthesiology and Critical Care Medicine, Université Laval, Québec City, Québec, Canada

7 Department of Medicine, Université Laval, Québec City, Québec Canada

8 Division of Critical Care, Children's Hospital of Eastern Ontario, Ottawa, Ontario, Canada

9 Division of Critical Care, Montreal Children's Hospital, McGill University Health Centre and Research Institute; Professor of Pediatrics, McGill University, Montreal, Canada

10 Department of Anesthesiology, Université de Sherbrooke, Sherbrooke, Québec 11 Transplant and Regenerative Medicine Centre, The Hospital for Sick Children, Toronto, Ontario, Canada

12 Carrefour de l'innovation, Centre de recherche du CHUM, Montréal, Québec, Canada

13 Canadian Donation and Transplant Research Program, Ottawa, Ontario, Canada

14 Division of Critical Care, Department of Medicine, Centre Hospitalier de l'Université de Montréal, Montreal, Quebec, Canada

Introduction: A presumed consent model of organ donation assumes all people of a defined geographic area consent for deceased organ donation unless they have expressed otherwise. Various forms of presumed consent exist ${ }^{1}$ that are often implemented to increase donation rates, but have yielded mixed results ${ }^{2-4}$. Literature reviews on the topic suggest that a presumed consent model is unlikely to increase donation rates unless done in a transparent manner with broad support of the public and healthcare professionals ${ }^{2,5}$. The current opinion of Canadian intensive care physicians towards presumed consent for organ donation is unknown. The last reported national survey of healthcare workers (including non-physicians) on the topic was published in $2006^{6}$, with $59 \%$ somewhat or strongly supportive of a presumed consent model.

Objective: Survey practicing adult and pediatric intensive care physicians on their attitudes towards a presumed consent model for deceased organ donation. Presumed consent questions were embedded in a larger survey on deceased donation practices in Canada.

Methods: We conducted a cross-sectional survey of Canadian intensive care physicians. We followed a standardized approach for design and conduct of selfadministered surveys ${ }^{7}$, including item generation and reduction using modified Delphi methods, survey validation for clarity, redundancy and comprehensiveness, and testretest reliability assessments. Our sampling frame included all intensivists practicing in an institution that may care for potential organ donors. Intensivists were identified by 
merging lists obtained in collaboration with Canadian Blood Services, the Canadian Critical Care Society and manual searches from publicly available sources (e.g. hospital websites). The survey was administered using an online platform (LimesurveyTM) from February 26 to March 26, 2018 with two e-mail reminders. Survey responses are reported using descriptive statistics.

Results: 550 potential respondents were identified, of which 21 did not meet inclusion criteria and 1 participant declined consent. The response rate was 49.7\% (263/529) and the completion rate $89.4 \%$ (235/263). Overall, $95.7 \%$ of respondents had a mostly positive or positive general opinion regarding organ donation in general. When asked if they would be in favor of a change towards a presumed consent model, $62.2 \%$ agreed or strongly agreed, $18.7 \%$ were neutral and $20 \%$ disagreed or strongly disagreed. $77 \%$ stated they believe a presumed consent model would increase organ donation rates, $25 \%$ expressed that such a model would increase the likelihood that they would approach a family to discuss organ donation.

Conclusion: In our survey, a majority expressed support for a presumed consent model. Though not asked in exactly the same manner or of the same population, the rate of support for presumed consent seems largely stable since a 2006 survey (6). A larger majority believed that a presumed consent model would increase organ donation rates, even though most respondents did not indicate they would be more likely to approach families and surrogates of patients who are potential donors. Further research is required to better understand attitudes and knowledge of if and how a presumed consent model should be implemented in Canada.

\section{References}

1. Zúñiga-Fajuri A. Increasing organ donation by presumed consent and allocation priority: Chile. Bull World Health Organ. 2015 Mar 1;93(3):199-202.

2. Rithalia A, McDaid C, Suekarran S, Norman G, Myers L, Sowden A. A systematic review of presumed consent systems for deceased organ donation. Health Technol Assess. 2009 May;13(26):1-134.

3. Domínguez J, Rojas JL. Presumed Consent Legislation Failed to Improve Organ Donation in Chile. TPS. Elsevier Inc; 2013 May 1;45(4):1316-7.

4. Young V, McHugh S, Glendinning R, Carr-Hill R. Evaluation of the Human Transplantation (Wales) Act: Impact Evaluation Report. 2017 Dec pp. 1-75.

5. Shepherd L, O'Carroll RE, Ferguson E. An international comparison of deceased and living organ donation/transplant rates in opt-in and opt-out systems: a panel study. BMC Med. 2014 Sep 24;12(1):1338-14.

6. Baker A, Rocker G, Shemie SD, Young K. Health Professional Awareness and Attitudes on Organ and Tissue Donation and Transplantation, a Report from the Canadian Council for Donation and Transplantation. 2006 Aug pp. 1-84.

7. Burns KEA, Duffett M, Kho ME, Meade MO, Adhikari NKJ, Sinuff T, et al. A guide for the design and conduct of self-administered surveys of clinicians. Canadian Medical Association Journal. 2008 Jul 29;179(3):245-52. 
The Cost Effectiveness of Mechanical Ventilation Using PAV+ Mode in Canada

Saunders, Rhodri' ${ }^{1}$; Bosma, Karen ${ }^{2,3}$

1 Health Economics, Coreva Scientific, Freiburg, Germany

2 Associate Professor of Medicine, Critical Care Medicine and Respirology, University of Western Ontario, London, Ontario, Canada

3 Critical Care, London Health Sciences Centre, London, Ontario, Canada

Background: Mechanical ventilation (MV) is an integral but expensive part of the intensive care unit (ICU). Events such as ventilator-associated pneumonia (VAP) and tracheotomy can further increase care costs. Optimizing MV provision could result in substantial cost savings and improved patient outcomes. Here, the health and cost outcomes of proportional assist ventilation plus (PAV+) mode are compared with pressure support ventilation (PSV), incorporating recent data including a randomizedcontrolled trial ${ }^{1}$, a meta-analysis ${ }^{2}$, and an ICU costing study ${ }^{3}$ published by Canadian institutions in 2018.

Methods: Data from clinical studies and randomized, controlled trials (RCTs) were used to inform event rates and patient risk in a computational model of a patient's care pathway once initiated on MV. Care settings include the ICU, general ward, and home. The model estimates patient outcomes (time on MV, days in ICU, days in hospital, and hospital discharge) and safety events, including the incidence of patient-ventilator asynchrony $>10 \%$, tracheotomy, VAP, nosocomial infections, spontaneous breathing trial (SBT) failure, and post-liberation hypoxemia. In-hospital and post-discharge mortality were taken from rates in peer-reviewed literature up to 3 years and from Canadian life tables thereafter. Patients, mean 65 years and $40 \%$ female, are on MV until a successful SBT and liberation. Time on MV is influenced by asynchrony $>10 \%$ and VAP. MV is either PSV or PAV+ mode, with differences between these interventions derived from the weighted mean outcomes of $6 \mathrm{RCTs} \mathrm{s}^{4-9}$. PAV+ mode was associated with less asynchrony, shorter time on MV (-1.62) and in the ICU, but longer time in hospital. Quality-adjusted life years (QALYs) used EQ-5D utilities. All event costs are expressed in 2017 CAD. The model's time horizon runs from 1-40 years with cost and health outcomes after the first year discounted at $1.5 \%$ per annum.

Probabilistic sensitivity analysis (PSA) provides estimates of outcome significance at the $95 \%$ level.

Results: The first-year cost of care was $\$ 54,253$ with PAV+, a saving of $\$ 6,200$ compared with PSV $(\$ 60,452)$. Outcomes reflected lower resource use with PAV+ mode. Of PAV+ patients, 0.28 QALYs were accumulated with $79 \%$ alive at the year end. For PSV, the respective values were 0.25 QALYs and $69 \%$ alive. Benefits were reduced mean time on MV (6.2 days) compared with PSV (8.4 days) and a lower rate of VAP. The 40-year cost of care $(\$ 160,543)$ was higher with PAV+ mode than with PSV $(\$ 153,610)$. Improvements in QALYs $(+0.96)$ and life expectancy $(+1.57$ years) were identified with PAV+ mode. Superior to PSV after 1-year, PAV+ was likely (100\%) to be considered cost-effective versus PSV at 40 years given a willingness-to-pay threshold of $\$ 50,000$ per QALY gained. PSA showed that increases in QALYs with $\mathrm{PAV}+$ mode were significant, but differences in costs were not. The mean cost per QALY gained was $\$ 7,380$ (median $\$ 4,955$ ). Results did not differ substantially if PAV+ mode had no impact on asynchrony, meta-analysis informed clinical effectiveness, or different cost sources were used. If future care costs were excluded, PAV+ mode was superior to PSV. Cost and outcomes drivers were time on mechanical ventilation and in the ICU.

Conclusions: Under modelled conditions, PAV+ mode increased patient quality of life and life expectancy. In the early years, healthcare costs decreased with use of PAV+ mode. Increased life expectancy led to higher long-term costs. 


\section{References}

1. Botha J, Green C, Carney I, Haji K, Gupta S, Tiruvoipati R. Proportional assist ventilation versus pressure support ventilation in weaning ventilation: a pilot randomised controlled trial. Crit Care Resusc. 2018;20(1):33-40

2. Vijayaraghavan TBK, Hamed S, Jain A, et al. Evidence Supporting Clinical Use of Proportional Assist Ventilation: A Systematic Review and Meta-Analysis of Clinical Trials. 2018:1-9. doi:10.1177/0885066618769021.

3. Evans J, Kobewka D, Thavorn K, D'Egidio G, Rosenberg E, Kyeremanteng K. The impact of reducing intensive care unit length of stay on hospital costs: evidence from a tertiary care hospital in Canada. Can J Anesth. 2018;65(6):627-635. doi:10.1007/s12630-018-1087-1

4. Bosma KJ, Read BA, Bahrgard Nikoo MJ, Jones PM, Priestap FA, Lewis JF. A pilot randomized trial comparing weaning from mechanical ventilation on pressure support versus proportional assist ventilation. Crit Care Med. 2016;44(6):1098-1108. doi:10.1097/CCM.0000000000001600.

5. Botha J, Green C, Carney I, Haji K, Gupta S, Tiruvoipati R. Proportional assist ventilation versus pressure support ventilation in weaning ventilation: a pilot randomised controlled trial. Crit Care Resusc. 2018;20(1):33-40.

6. Sasikumar S, Shanbhag V, Shenoy A, Unnikirshanan R. Comparison of pressure support and proportional assist ventilation plus for weaning from mechanical ventilation in critically ill patients. Indian J Crit Care Med. 2013;17(May 2016):34.

7. Elganady AA, Beshey BN, Abdelaziz AAH. Proportional assist ventilation versus pressure support ventilation in the weaning of patients with acute exacerbation of chronic obstructive pulmonary disease. Egypt J Chest Dis Tuberc. 2014;63(3):643650. doi:10.1016/j.ejcdt.2014.04.001.

8. Xirouchaki N, Kondili E, Vaporidi K, et al. Proportional assist ventilation with loadadjustable gain factors in critically ill patients: Comparison with pressure support. Intensive Care Med. 2008;34(11):2026-2034. doi:10.1007/s00134-008-1209-2.

9. Aguirre-Bermeo H, Bottiroli M, Italiano S, et al. Ventilación con presión de soporte y ventilación proporcional asistida durante la retirada de la ventilación mecánica. Med Intensiva. 2014;38(6):363-370. doi:10.1016/j.medin.2013.08.003. 
The transition of International Medical Graduate (IMG) fellows into the ICU Team: Identity Gain or Loss?

Mecklenburg, $A^{1}$; Najeeb $U^{2}$; Rose $L^{3}$; Lingard L ${ }^{4}$; Piquette $D^{1}$

1 Interdepartmental Division of Critical Care, University of Toronto, Ontario, Canada

2 Sunnybrook Health Sciences Centre, Department of Medicine, University of Toronto, Toronto, Ontario, Canada

3 Sunnybrook Health Sciences Centre, Lawrence S. Bloomberg Faculty of Nursing and Interdepartmental Division of Critical Care Medicine, University of Toronto, Toronto, Ontario, Canada

4 Centre for Education Research and Innovation, Schulich School of Medicine and Dentistry, Western University, London, Ontario, Canada

Introduction: IMGs play an integral part in many health care systems worldwide. ${ }^{1}$ When they pursue clinical training in a different country, IMGs face the need to adapt to an unfamiliar working environment. ${ }^{2,3}$ The practice of critical care is complex, time-sensitive and highly dependent on inter-disciplinary teamwork. In fact, effective team work has been tied to better ICU patient outcomes. ${ }^{4}$ Understanding the "rules of the game" within an ICU team may represent a unique challenge for IMGs starting a critical care fellowship. The inability or delayed ability to adapt to their new training environment may be problematic for the trainees and for patient care. In addition, the unique role of a critical care fellow within an ICU team is foreign to many IMGs who trained in countries without fellowship programs. IMGs may initially not fully grasp the dual, sometimes conflictual nature (supervisor-supervisee; leader-follower) of their new professional role.

Objectives: This study aims to better understand how IMGs experience their transition within multidisciplinary ICU teams during their critical care fellowship. More specifically, this project explores: 1) IMG fellows' perceptions of their role within the multiprofessional ICU team; 2) challenges encountered by IMG fellows as they transition into their new professional role; 3) strategies used by IMG fellows to overcome these challenges. We explored how known theories on professional transition apply to the ICU environment with the ultimate goal of developing evidence-informed initiatives to support IMG fellows and ICU teams during the early transition period in the fellowship. Methods: In this qualitative single center study, we conducted 16 individual interviews with IMG fellows who were enrolled in the adult critical care fellowship program of the University of Toronto. Fellow's perceptions of their role and transition within the ICU team were explored and iteratively analyzed through a constructivist grounded theory approach, including purposive sampling, inductive coding, constant comparison and inter-disciplinary theoretical construction of a model.

Results: Participants came from Europe, Central/South America, North America, Australia, Asia and the Middle East. Our analysis revealed that most of the participants had a limited understanding of the role of a fellow. For many fellows, this uncertainty translated into an initial lack of confidence in their ability to fulfill their role. The North American model of multidisciplinary ICU team (composition and roles) was perceived as a huge challenge. As they tried to work within the ICU team, participants reported experiencing many losses: autonomy, appreciation, efficiency, skills, and personal work standards. Fellows maneuvered this period of transition by relying on honed clinical skills, building trust with ICU team members, changing attitudes towards teamwork, or seeking social support. Others described a period of resistance where they actively challenged the system, the team or their colleagues.

Conclusion: Our preliminary findings show that IMGs who have to transition into the role of a fellow within an inter-disciplinary ICU team encounter unique challenges.

Uncertainties experienced at the beginning of the fellowship lead to a dynamic process of collapse and re-construction of their professional identity. Such findings will directly 
inform the design of new strategies to facilitate IMGs' transition into North American ICU fellowships.

\section{References}

1. OECD: International Migration Outlook 2015. OECD Publishing 2015; 1-378.

2. Zulla R, Baerlocher MO, Verma S: International medical graduates (IMGs) needs assessment study: comparison between current IMG trainees and program directors. BMC Medical Education 2008; 8:322-5.

3. Wong A, Lohfeld L: Recertifying as a doctor in Canada: international medical graduates and the journey from entry to adaptation. Med Educ 2007; 42:53-60.

4. Wheelan S: The link between teamwork and patients' outcome in Intensive Care Units. Journal of Critical Care 2004; 1-9 
Transitions in Critical Care: Evaluating the Implementation of a Handover Tool for Postoperative Cardiac Surgery Patients

Denomme, Justine $^{1}$; Isac, Michelle ${ }^{1}$; Syed, Summer ${ }^{1}$; Yu, Janice ${ }^{2}$; Centofanti, John ${ }^{3}$ 1 Department of Anesthesia, McMaster University, Hamilton, Ontario, Canada 2 Undergraduate Medical Program, McMaster University, Hamilton, Ontario, Canada 3 Department of Anesthesia and Department of Critical Care, McMaster University, Hamilton, Ontario, Canada

Introduction: Accurate and efficient handover is critical in the transfer of postoperative surgical patients by anesthesiology to the cardiac intensive care unit (CVICU). A systematic review of postoperative handover identified an association between the quality of patient handover and patient harm ${ }^{1}$. One strategy to improve handover and prevent adverse events is the application of checklists to standardize communication and minimize omissions ${ }^{2}$. Our academic centre recently implemented a standardized handover tool for the transfer of postoperative cardiac surgery patients to the CVICU. Objectives: To assess completion of a novel handover tool 6-months postimplementation, and elicit attitudes and perceptions of anesthesiologists and members of the CVICU team about perioperative patient handover.

Methods: Ethics review was completed via the local REB. Chart review began 6months post-implementation of the handover tool and included 373 consecutive patients who underwent cardiac surgery over a 3-month period. Completion of the 59 parameters on the handover tool was assessed for adherence, and compared to the anesthetic record and preoperative investigations to assess for quality and accuracy of information. Using an online database, a self-administered survey was created regarding perioperative handover of CVICU patients. The survey was pilot tested and broadly disseminated to all cardiac anesthesiologists as well as healthcare professionals working in the CVICU. Questions were designed using a 5-point Likert scale with opportunity for free text commentary.

Results: 357 (96\%) of the charts reviewed included a copy of the handover tool. The parameters most frequently completed ( $>80 \%$ of charts) included medical history, airway management, arterial lines, cardiac bypass duration, drug dosing, and the transferring anesthesiologist's name. Documentation regarding pacing, recent bloodwork, central lines, peripheral IVs, and time of transfer were completed in less than $80 \%$ of charts. The response rate to the survey was 111 of 154 stakeholders $(72 \%) .72 \%$ of respondents felt the handover tool improved the handover process and $62 \%$ felt it improved handover efficiency. No respondents felt the tool worsened handover. The majority felt the tool improved the transfer of important details of intraoperative care. The tool also enhanced verbal communication by anesthesiology trainees, standardized handover among anesthesia providers, and allowed access to important perioperative details after handover was completed. Barriers to success of the handover tool included a lack of awareness of its existence and nominal completion. Barriers to the effectiveness of overall patient transition included: insufficient clearance of physical space during patient transfer, lack of attendance of the cardiac surgeon and ICU attending during handover, and difficulty hearing verbal handover at the bedside.

Conclusions: The introduction of a handover tool for cardiac surgical patients has improved handover from the perspective of its users, and continues to be used with promising adherence 6-months post-implementation. It is a useful resource detailing important patient and surgical information, and continues to be used even after verbal handover was completed. Barriers to effective transition of patients into the ICU with high-quality handover were identified and can be targeted for continuous quality improvement interventions. 


\section{References}

1. Segall N, Bonifacio AS, Schroeder RA, et al. Can we make postoperative patient handovers safer? A systematic review of the literature. Anesth Analg. 2012;115(1):102-115.

2. Agarwal HS, Saville BR, Slayton JM, et al. Standardized postoperative handover process improves outcomes in the intensive care unit: a model for operational sustainability and improved team performance. Crit Care Med. 2012;40(7):21092115. 
Trends and Outcomes in Mechanically Ventilated Cirrhotic Patients

Cheung, Kyle ${ }^{1}$; Mailman, Jonathan ${ }^{2}$; Crawford, Jennifer ${ }^{3}$; Sy, Eric ${ }^{4}$

1 University of Saskatchewan College of Medicine, Saskatoon, Saskatchewan, Canada

2 Regina Qu'Appelle Health Region, Regina, Saskatchewan, Canada

3 Regina Qu'Appelle Health Region, Regina, Saskatchewan, Canada

4 Regina Qu'Appelle Health Region, Regina, Saskatchewan, Canada

Background: Cirrhotic patients who receive mechanical ventilation are known to have exceptionally poor outcomes, with a reported mortality rate in excess of $35 \%^{1,2}$. In this study, we analyzed Healthcare Cost and Utilization Project (HCUP) National Inpatient Study (NIS) database to learn more about this high-risk population.

Objectives: The purpose of this study was to examine the impact of various comorbidities and/or interventions on mortality in this patient subpopulation. Further, we examined the impact of these factors on hospital length of stay (LOS) as well as the total financial cost per hospitalization.

Methods: A retrospective analysis was performed on the HCUP NIS database from years 2005-2014. To select our subpopulation, the following inclusion criteria were applied: a) over the age of 18 years; b) assigned a diagnosis of cirrhosis (International Classification of Diseases 9th edition [ICD-9] code 571) and c) assigned an ICD-9 procedure code for mechanical ventilation (96.7). Multiple regression was used to assess the association between specific patient factors on in-hospital mortality, LOS and total hospital charges while adjusting for age, sex, race, Charlson comorbidity category, primary payer source, median income in the patient's residential area, elective versus emergency admission, location, size, and teaching status.

Results: In the general mechanically ventilated inpatient population, in-hospital mortality was $27.7 \%$ (95\% confidence interval [Cl] $26.7 ; 28.7 \%)$ and amongst mechanically ventilated cirrhotics was $41.8 \%(95 \% \mathrm{Cl} 39.9 ; 43.7 \%)$. Multivariate analysis showed several comorbidities that were associated with an increased likelihood of in-hospital mortality, including hepatorenal syndrome, odds ratio (OR) 3.24 $(95 \% \mathrm{Cl} 3.02$; 3.47); sepsis, OR $3.23(95 \% \mathrm{Cl} 3.11$; 3.37) and renal failure, OR= 2.84 $(95 \% \mathrm{Cl} 2.74 ; 2.94)$. The average LOS in the mechanically ventilated population was $14.9(95 \% \mathrm{Cl} 14.7 ; 15.2)$, and in our sub-population was 12.9 days $(95 \% \mathrm{Cl} 12.7 ; 13.2)$. Certain procedures were associated with increased hospital LOS, such as receiving a tracheostomy, 24.8 days $(95 \% \mathrm{Cl} 23.3 ; 26.3)$; liver transplant, 21.1 days $(95 \% \mathrm{Cl} 17.6$; $24.7)$; and hemodialysis, 5.0 days $(95 \% \mathrm{Cl} 4.5$; 5.5). The average cost per hospitalization in mechanically ventilated patients without cirrhosis was $\$ 148,792$ (95\% CI \$145,039; 152,546) in our sub-population was \$152,653 (95\% Cl \$146,981; $158,325)$.Total hospital charges were also strongly influenced by procedures such as receiving a liver transplant, $\$ 440,493(95 \% \mathrm{CI} \$ 390,030 ; 490,957)$; tracheostomy, $\$ 290,898$ (95\% Cl $\$ 272,361 ; 309,436)$ and hemodialysis, $\$ 100,271$ (95\% Cl \$89,940; 110,602).

Conclusions: There are several comorbidities associated with increased mortality amongst mechanically ventilated cirrhotic patients, including hepatorenal syndrome, sepsis, and renal failure. Procedures on this high-risk population were generally associated with improved mortality rates but significantly longer hospital stays and higher financial costs. Our results reinforce the notion that mechanically ventilated cirrhotics are a high-risk and expensive population with generally poor outcomes. Patients with impaired renal function and suspected sepsis should be treated carefully as they have an even greater risk of in-hospital mortality. Our work outlines a need for further investigation in order to better manage and treat this subpopulation of patients. 


\section{References}

1. Shellman RG, Fulkerson WJ, DeLong E, Piantadosi CA. Prognosis of patients with cirrhosis and chronic liver disease admitted to the medical intensive care unit. Crit Care Med 1988;16:671-678.

2. Singh N, Gayowski T, Wagener MM, Marino IR. Outcome of patients with cirrhosis requiring intensive care unit support: prospective assessment of predictors of mortality. J Gastroenterol 1998;33:73-79. 
Understanding the Influence of Inter-Professional Relational Networks within Organ Donation Programs in Ontario: A Research Protocol and Preliminary Results

Silva e Silva, Vanessa ${ }^{1,2}$; Tranmer, Joan E. ${ }^{1}$; Schirmer, Janine ${ }^{2}$; Roza, Bartira D’Aguiar $^{2}$; Almost, Joan ${ }^{1}$; Shafer, Markus ${ }^{3}$; Dhanani, Sonny ${ }^{4}$

1 School of Nursing, Queen's University, Kingston, Ontario, Canada

2 School of Nursing, Federal University of São Paulo, São Paulo, Brazil

3 Department of Sociology, University of Toronto, Toronto, Canada

4 Department of Medicine, University of Ottawa, Ottawa, Canada

Introduction: To optimize organ donation performance the nature and impact of complex factors need to be understood. Some factors are well known and might not be modifiable (contra-indications for transplant, age limit of organ donor, etc), but others (collaboration and relationships) that contribute to variations among similar hospitals in the same province, are poorly understood.

Objectives: The overall aim of this research is to understand the nature and impact of relationships within the organizational context of organ donation programs in Ontario. Methods: We will employ an exploratory, prospective, diagnostic study consisting of three approaches: (1) to describe the characteristics of social networks of health care professionals of the organ donation programs (Social Network Analysis) and (2) to describe the organizational attributes and processes of on organ donation programs (Multiple Case-Study), and (3) to compare the influence of social networks and organizational attributes on the performance of organ donation programs (Network Comparison). The study sites will include Ontario hospitals designated as type A based on trauma centre level (Public Hospitals Act classification); and hospitals partners of the Ontario's Organ Donation Organization. At least five sites will be purposely selected to capture the greatest variability of settings possible.

Results: We have initiated the study, signed a data protection agreement with Ontario's Organ Donation Organization, and we are now on the first site collecting data.

Conclusion: Understanding the influences of informal networks on organ donation outcomes is essential to the development and informing of interventions to optimize performance. This research will increase organ donation rates in Ontario by providing to the scientific community a novel measure of organ donation programs' performance and by identifying successful collaboration paths during organ donation processes. We will present the development process of the research as well as preliminary findings. 


\section{SOCIAL NETWORK ANALYSIS}

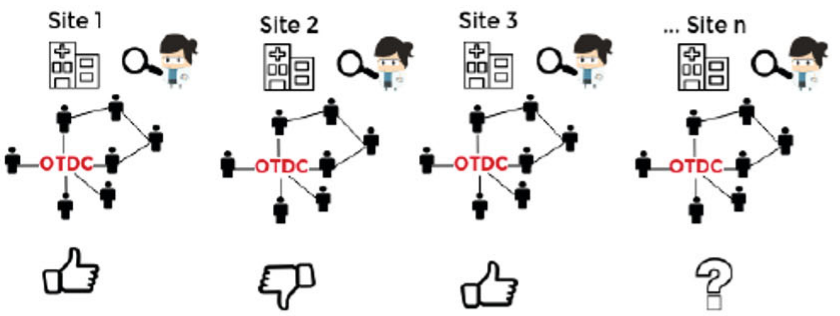

Figure 2. Data collection and analysis procedures

\section{MULTIPLE CASE-STUDY}
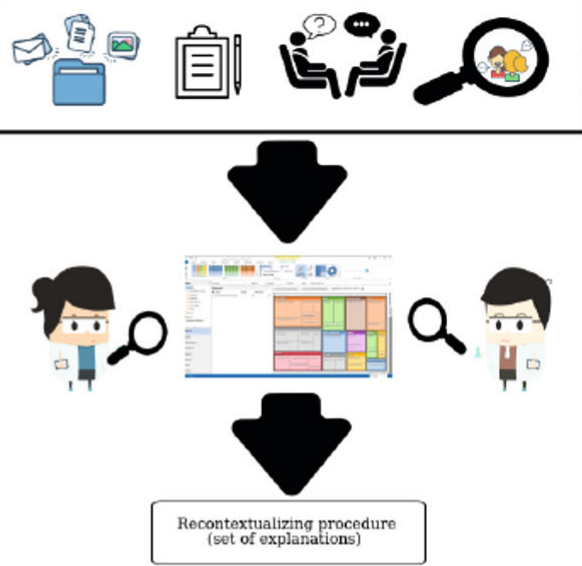

Figure 2. Case-study data analysis

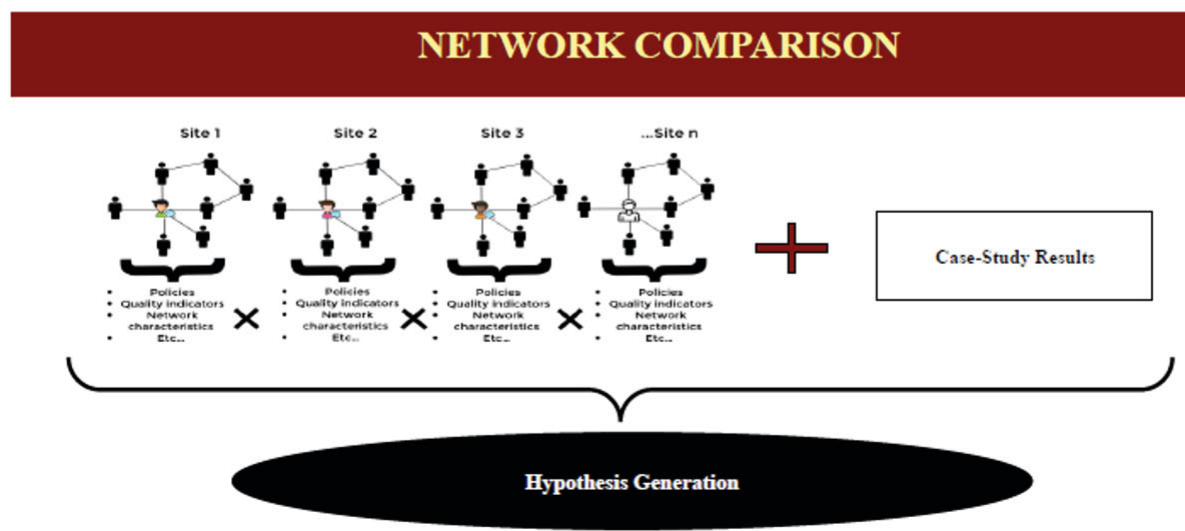

Figure 3. Network comparison data analysis 
Using Non-Invasive Techniques to Explore Personalized Cerebral Perfusion Targets in Critically III Patients

Lee, Kevin F.H. ${ }^{1}$; Wood, Michael D. ${ }^{2}$; Maslove, David M. ${ }^{3,4}$; Muscedere, John G. ${ }^{3}$; Boyd, J. Gordon $2,3,4$

1 School of Medicine, Queen's University, Kingston, Ontario, Canada

2 Centre for Neuroscience Studies, Queen's University, Kingston, Ontario, Canada

3 Department of Critical Care Medicine, Queen's University, Kingston, Ontario, Canada

4 Department of Medicine, Queen's University, Kingston, Ontario, Canada

Introduction/Background: Cerebral autoregulation (CA) is a vascular reflex mechanism that responds to changes in systemic blood pressure in order to maintain constant cerebral blood flow. CA dysfunction during cardiac surgery, intracerebral hemorrhage and traumatic brain injury is associated with negative outcomes ${ }^{1-3}$. Less is understood about CA function in the setting of critical illness. We recently showed that cerebral oxygenation during critical illness was associated with delirium, which may be related to CA dysfunction ${ }^{4}$. We analyzed CA function in critically ill patients during the first 24-72 hrs in the ICU using near-infrared spectroscopy (NIRS)-based cerebral oximetry $^{5-9}$. We developed a novel approach to derive mean arterial pressure (MAP) targets that may help optimize cerebral perfusion in individual patients.

Objective: To identify putative MAP targets in critically ill patients using NIRS-based cerebral oximetry.

Methods: MAP and rSO2 were recorded simultaneously for up to the first 72 hours in ICU ( $n=40$ patients) by arterial catheter and NIRS, respectively. The cerebral oximetry index (COx) was computed as the moving Spearman correlation between MAP and rSO2 (Figure 1, 2). Positive COx values were taken to reflect CA dysfunction while near-zero or negative $\mathrm{COx}$ values reflect intact $C A^{5-7}$. We asked whether certain MAP values were associated with intact vs dysfunctional $C A$, and so binned the MAP values by COx (in bins of 0.05 , from -1 to +1 ). This approach builds on previous research to identify optimal cerebral perfusion targets ${ }^{5-11}$.

Results: COx signals were variable between patients, with some patients exhibiting greater periods of CA dysfunction than others. In a separate parallel analysis, we found that CA dysfunction was associated with the subsequent development of delirium (data not shown). After sorting MAP values into their associated $\mathrm{COx}$ bins, the resulting plots adopted four general patterns: negative slope $(n=18 / 40$ patients, Figure $3 A)$, positive slope (2/40, Figure 3B), flat (13/40, Figure 3C) and complex (7/40, Figure 3D). Plots with either positive or negative slopes clearly showed certain MAP 'danger' zones that were associated with CA dysfunction (high COx values), as well as relative 'safe' zones (Figure 3A-B). Danger zones and safe zones were separated by $8 \pm 4 \mathrm{mmHg}$ on average, with average MAPs of $76 \pm 8 \mathrm{mmHg}$ and $82 \pm 10 \mathrm{mmHg}$, respectively. MAP targets were not readily discernable in plots with flat or complex morphologies from the other half of our study subjects (Figure 3C-D). The basis for the different plot morphologies is presently unclear, but may be related to the patients' position on the cerebral autoregulation curve.

Conclusions: We outlined an approach for identifying patient-specific MAP targets using NIRS-based cerebral oximetry. This strategy may help preserve cerebral blood flow and reduce the risk of negative neurocognitive outcomes. Our data demonstrate how patient-specific parameters can inform the identification of MAP targets on a caseby-case basis, and suggest that the classic MAP target of $65 \mathrm{mmHg}$ during critical illness may need revisiting ${ }^{12-13}$. The ability to identify physiological targets in individual patients in real-time may allow precise personalized interventions that have the potential to improve outcomes for survivors of critical illness. 
Figure 1 - Computing the Cerebral Oximetry Index (COx)

Time-resolved rSO2-MAP Correlation Analysis

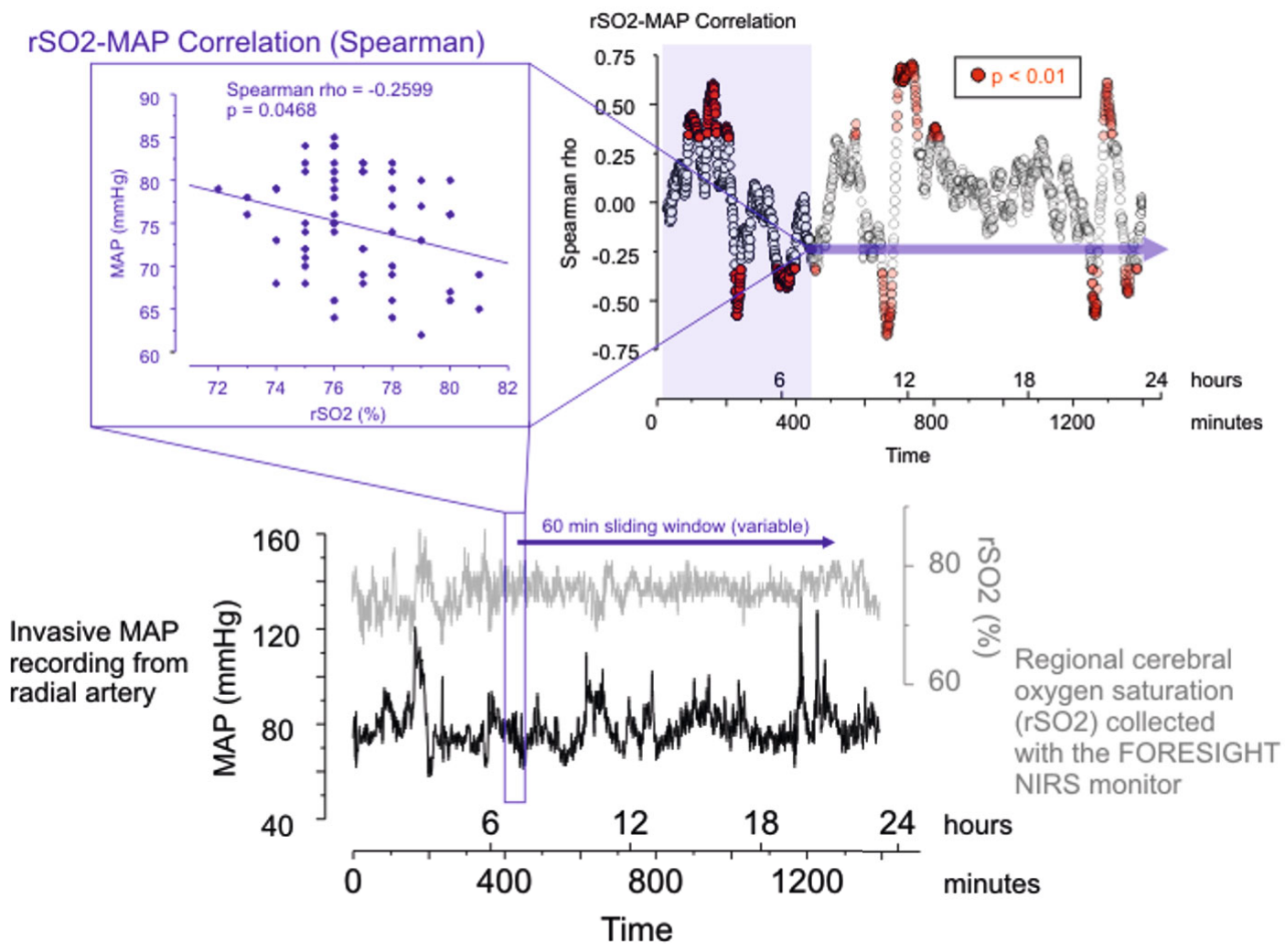

MAP recordings from the radial artery and non-invasive measurements of $\mathrm{rSO}_{2}$ by NIRS were obtained during the first 24-72 hours in the ICU. COx was iteratively calculated as the moving Spearman correlation coefficient (rho) between $\mathrm{rSO}_{2}$ and MAP with a given moving window length in one-minute steps. A 60 min moving window is shown here. Statistically significant COx values $(p<0.01)$ are shown in red in the upper right panel. 
Figure 2 - Effect of Moving Window Length on COx Time Series

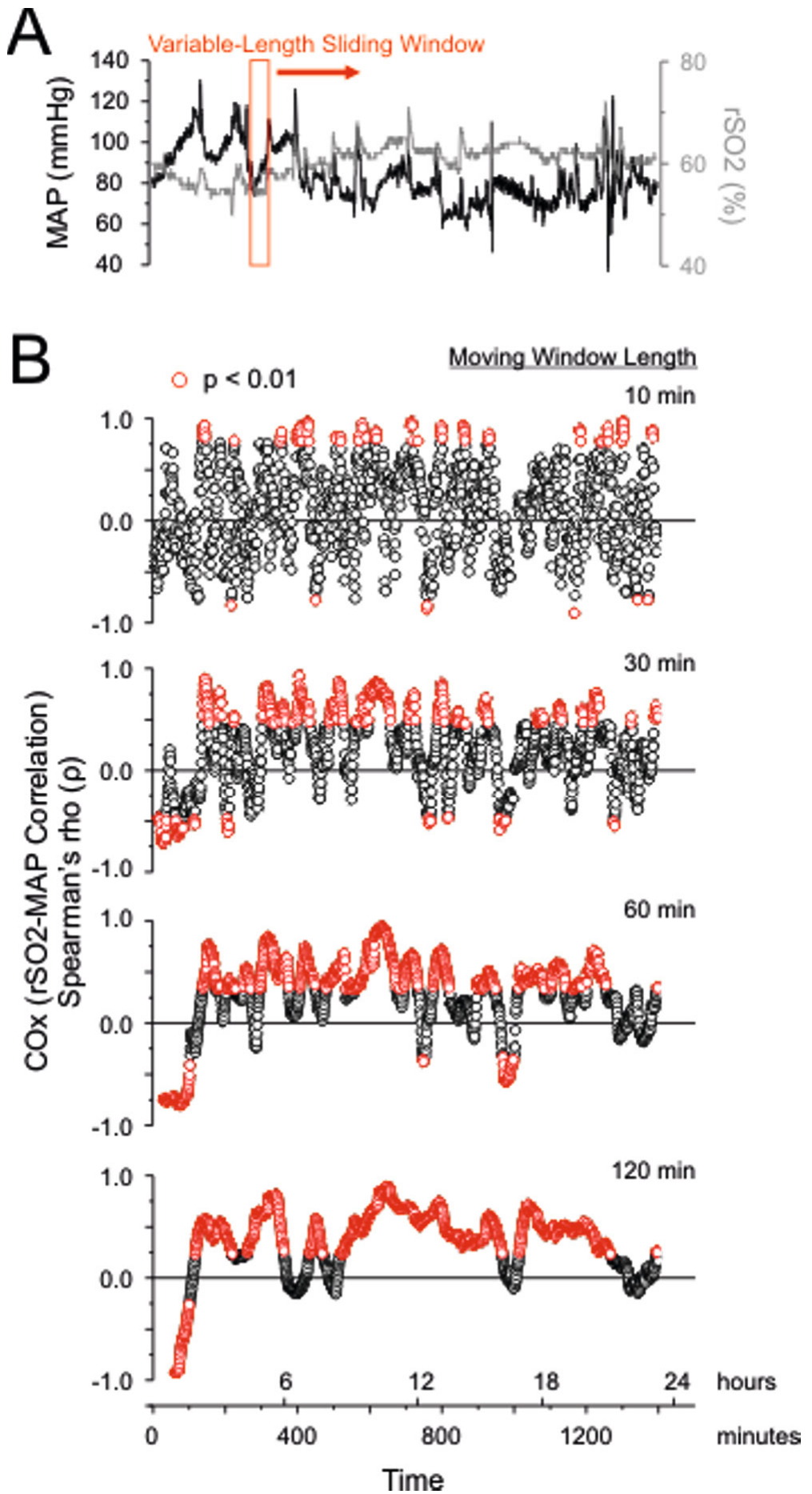

(A) MAP and $\mathrm{rSO}_{2}$ data recorded from a patient during the first 24 hours in the ICU.

(B) COx time series computed from data shown in (A), with 10, 30, 60 and 120 minute moving windows. COx correlations where $p<0.01$ are shown in red. 


\section{Figure 3 - Identification of Optimal MAP Targets Using NIRS-Based}

\section{Cerebral Oximetry}

A

(I)
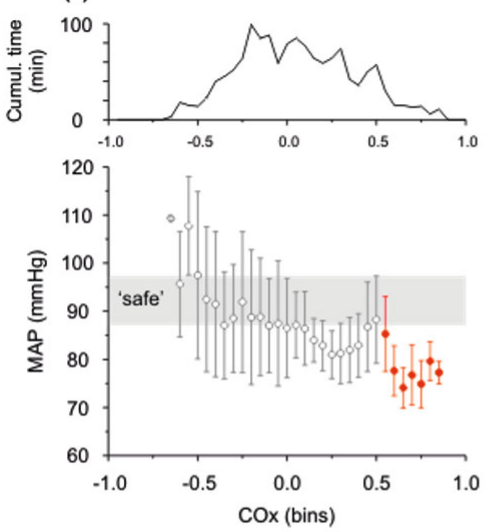

(III)
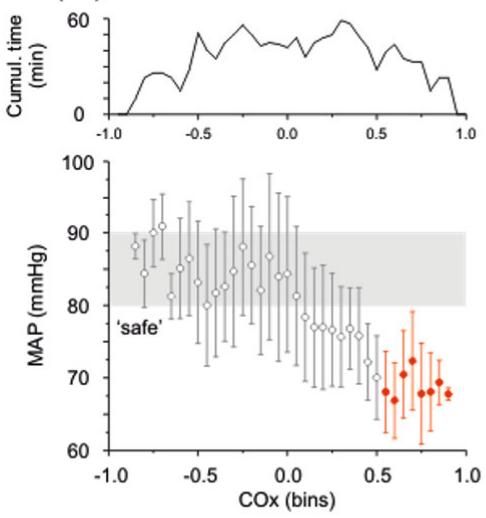

(II)
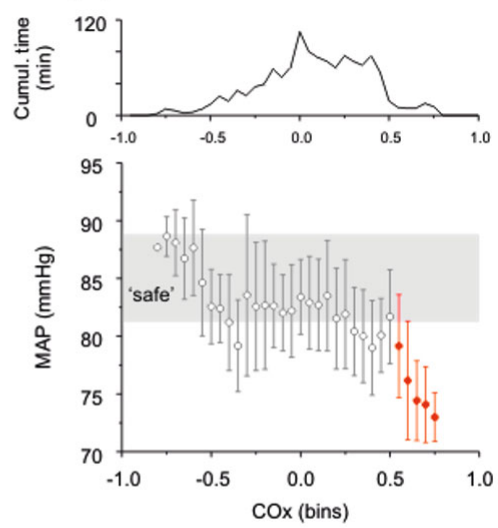

(IV)
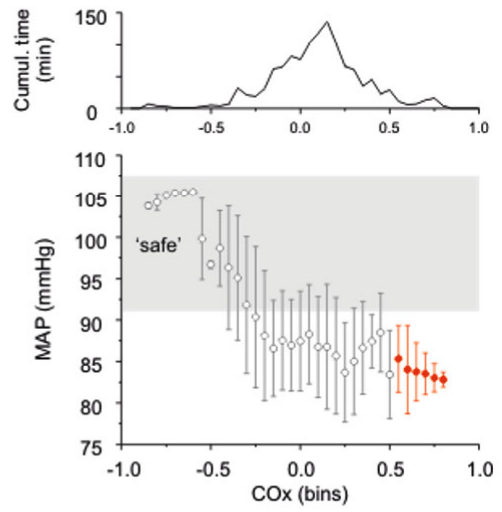
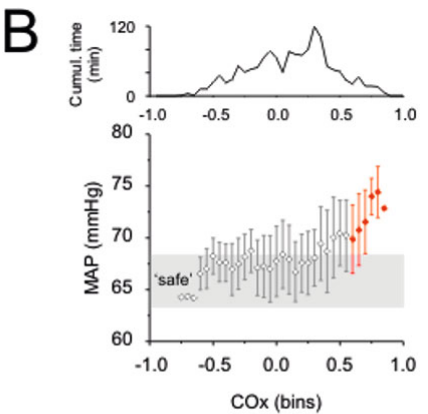

C
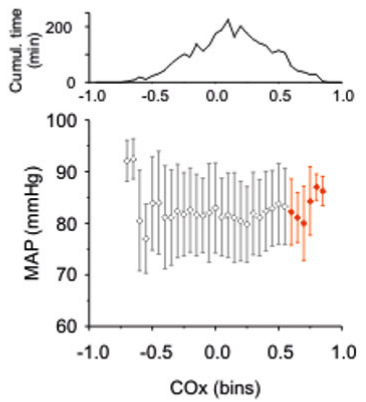

$\mathrm{D}$
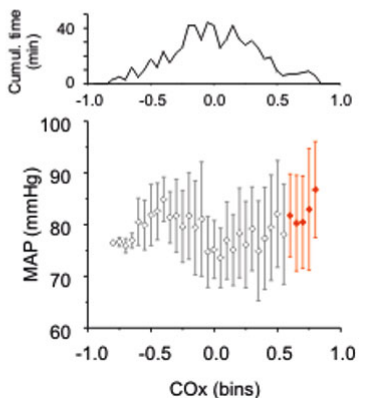

MAP values were sorted into their associated COx bins (bins of 0.05 , from -1 to +1 ). The resulting MAP-vs-COx plots adopted four basic patterns: (A) Negative slope, (B) Positive slope, (C) Flat, (D) Complex. Above each plot is the cumulative time distribution across COx bins. Red data points correspond to $\mathrm{COx}$ values representing CA dysfunction (COx, $\mathrm{p}<0.0001)$. MAP ranges associated with relative 'safe' zones were observed in plots with negative or positive slope (A, B). Safe zones for MAP were less clear in flat or complex plots (C, D).

\section{References}

1. Pandharipande PP, Girard TD, Jackson JC, Morandi A, Thompson JL, Pun BT, et al. Long-term cognitive impairment after critical illness. The New England journal of medicine. 2013 Oct 3;369(14):1306-16. PubMed PMID: 24088092. Pubmed Central PMCID: 3922401.

2. Rivera-Lara L, Zorrilla-Vaca A, Geocadin R, Ziai W, Healy R, Thompson R, et al. Predictors of Outcome With Cerebral Autoregulation Monitoring: A Systematic Review and Meta-Analysis. Critical care medicine. 2017 Apr;45(4):695-704. PubMed PMID: 28291094. 
3. Caldas JR, Haunton VJ, Panerai RB, Hajjar LA, Robinson TG. Cerebral autoregulation in cardiopulmonary bypass surgery: a systematic review. Interactive cardiovascular and thoracic surgery. 2018 Mar 1;26(3):494-503. PubMed PMID: 29155938

4. Wood MD, Maslove DM, Muscedere JG, Day AG, Boyd JG. Low brain tissue oxygenation contributes to the development of delirium in critically ill patients: A prospective observational study. Journal of critical care. 2017 Oct;41:289-95. PubMed PMID: 28668768.

5. Brady KM, Lee JK, Kibler KK, Smielewski P, Czosnyka M, Easley RB, et al. Continuous time-domain analysis of cerebrovascular autoregulation using nearinfrared spectroscopy. Stroke. 2007 Oct;38(10):2818-25. PubMed PMID: 17761921. Pubmed Central PMCID: 2377358.

6. Budohoski KP, Czosnyka M, Smielewski P, Varsos GV, Kasprowicz M, Brady KM, et al. Cerebral autoregulation after subarachnoid hemorrhage: comparison of three methods. Journal of cerebral blood flow and metabolism : official journal of the International Society of Cerebral Blood Flow and Metabolism. 2013 Mar;33(3):44956. PubMed PMID: 23232948. Pubmed Central PMCID: 3587818.

7. Sekhon MS, Smielewski P, Bhate TD, Brasher PM, Foster D, Menon DK, et al. Using the relationship between brain tissue regional saturation of oxygen and mean arterial pressure to determine the optimal mean arterial pressure in patients following cardiac arrest: A pilot proof-of-concept study. Resuscitation. 2016 Sep;106:120-5. PubMed PMID: 27255957.

8. Rivera-Lara L, Zorrilla-Vaca A, Healy RJ, Ziai W, Hogue C, Geocadin R, et al. Determining the Upper and Lower Limits of Cerebral Autoregulation With Cerebral Oximetry Autoregulation Curves: A Case Series. Critical care medicine. 2018 May;46(5):e473-e7. PubMed PMID: 29419556. Pubmed Central PMCID: 5899039.

9. Brady K, Joshi B, Zweifel C, Smielewski P, Czosnyka M, Easley RB, et al. Real-time continuous monitoring of cerebral blood flow autoregulation using near-infrared spectroscopy in patients undergoing cardiopulmonary bypass. Stroke. 2010 Sep;41(9):1951-6. PubMed PMID: 20651274. Pubmed Central PMCID: 5544901.

10. Schramm P, Klein KU, Falkenberg L, Berres M, Closhen D, Werhahn KJ, et al. Impaired cerebrovascular autoregulation in patients with severe sepsis and sepsisassociated delirium. Critical care. 2012 Oct 4;16(5):R181. PubMed PMID: 23036135. Pubmed Central PMCID: 3682283.

11. Aries MJ, Czosnyka M, Budohoski KP, Steiner LA, Lavinio A, Kolias AG, et al. Continuous determination of optimal cerebral perfusion pressure in traumatic brain injury. Critical care medicine. 2012 Aug;40(8):2456-63. PubMed PMID: 22622398.

12. Joannidis M, Druml W, Forni LG, Groeneveld ABJ, Honore PM, Hoste E, et al. Prevention of acute kidney injury and protection of renal function in the intensive care unit: update 2017 : Expert opinion of the Working Group on Prevention, AKI section, European Society of Intensive Care Medicine. Intensive care medicine. 2017 Jun;43(6):730-49. PubMed PMID: 28577069. Pubmed Central PMCID: 5487598.

13. Rhodes A, Evans LE, Alhazzani W, Levy MM, Antonelli M, Ferrer R, et al. Surviving Sepsis Campaign: International Guidelines for Management of Sepsis and Septic Shock: 2016. Intensive care medicine. 2017 Mar;43(3):304-77. PubMed PMID: 28101605. 
Ventilator-Associated Pneumonia or Ventilator-Associated Event?

Laporta, Denny ${ }^{1,2}$; Peretz-Larochelle, Maude ${ }^{1} ;$ Abdou, Eman ${ }^{1}$

1 Adult Critical Care, Jewish General Hospital, Montreal, Quebec, Canada

2 Faculty of Medicine, McGill University, Montreal, Quebec, Canada

Introduction: Despite an increasing literature suggesting the poor performance of VAP as a quality measure of the care of mechanically-ventilated patients (), the only Accreditation Canada standard pertaining specifically to these patients relates to VAP prevention and treatment. The concept of Ventilator-Associated Event (VAE), introduced by the $\mathrm{CDC} / \mathrm{NHSN}$ as a more suitable measurement in the evaluation of clinical care and outcome in these patients.

Objective: We wished to 1) test the hypothesis that VAE identification adds value to current VAP surveillance data in a mixed med-surg ICU population, 2) to extrapolate from these data the number of potentially-missed VAEs during this time period.

Methods: We retrospectively reviewed the charts of ICU patients previously identified with VAP between January 2009 and September 2016 and applied the CDC/NHSN criteria for VAE occurring 7 days before or after their designated VAP date. Data were merged into a preexisting ICU database. We first compared clinical/outcome data between patients intubated/ventilated $(I / V) \leq 2$ days (d) with those where and those I/V $>2 \mathrm{~d}$ (Table 1). We then compared the same parameters for 1) VAP cases with those patients without VAP but similarly I/V $>2 \mathrm{~d}$, as well as 2) VAP cases based on whether they also met VAE criteria (Table 2). Finally, we compared our data with those from a similar published Canadian ICU population in order to estimate the number of "missed" cases of VAE in our population.

Results: Of 7919 ICU admissions, 1758 (22\%) were I/V >2d, of which 143 cases (8\%) of VAP were identified; 47/143 (33\%) had coexisting VAE (VAC 36\%, iVAC 30\%, "VAEVAP" 34\%). Patients I/ $>2 d$ comprised a sicker, older population than those I/ $\mathrm{V} \leq 2 \mathrm{~d}$ (Table 1) with differences in case mix and pre-ICU origin, increased LOS and mortality (icu \& hosp), prop of ICU/hosp-days, ventilator utilization and ICU

readmission. Patients with VAP showed differences in case mix and pre-ICU origin when compared those without VAP (Table 2), as well as increased LOS (icu \& hosp), $\%$ of ICU/hospital-days, \% of admissions with LOSicu $>7 \mathrm{~d}$ and utilization of ventilator days. VAP patients with coexisting VAE were similar to those without VAE, except for a larger number of transfers to another ICU/institution. This may explain the higher \% of ICU/hospital-days and the trend towards decreased ICU readmission rate and hospital LOS. NB only $1 / 3$ of VAE patients had concurrent VAE-derived VAP Comparison with VAP and VAE data from a similar Canadian ICU population showed a similar distribution of ventilator-related parameters. We estimated from this comparison that at least 117 cases of VAE were missed by virtue of not having concurrent VAP identification.

Conclusions: In our ICU patient population, the

- Significant differences in resource utilization and clinical outcomes were observed when comparing patients ventilated $>2$ VS $\leq 2$ days or whether VAP positive vs negative.

- However patients with VAP who had concurrent VAE were similar to those without VAE except for a greater proportion of patients transferred to another institution/ICU. - We estimate that 117 cases of VAE were potentially missed, which limits evaluative comparison between VAE and traditionally-identified VAP. It seems likely that these parameters reflect different patient mix, and that quality reviews should consider them as complementary information. 
Table 1

\begin{tabular}{|c|c|c|}
\hline & VENT $\leq 2$ days & VENT $>2$ days \\
\hline \# admissions & 6161 & 1758 \\
\hline Age (average)* & $66(14)$ & $69(14)$ \\
\hline$\%>$ age $80^{\circ}$ & $15.60 \%$ & $24 \% *$ \\
\hline LOS ICU & $2.4(3.2)$ & $18.5(47)^{*}$ \\
\hline LOS HOSPITAL & $20.9(53.3)$ & $49.9(82.5)^{*}$ \\
\hline$\%$ ICU/Hospital Days & $12.50 \%$ & $41.3 \%^{*}$ \\
\hline \multicolumn{3}{|l|}{ Origin intrahospital } \\
\hline ER/Other ICU & $15 \%$ & $34 \% *$ \\
\hline OR/PACU & $64 \%$ & $32 \% *$ \\
\hline Ward & $21 \%$ & $34 \% *$ \\
\hline \multicolumn{3}{|l|}{ Casemix } \\
\hline MED & $29 \%$ & $52 \% *$ \\
\hline SURG & $15 \%$ & $32 \% *$ \\
\hline CARD SURG & $56 \%$ & $34 \% *$ \\
\hline Readmission & $6 \%$ & $13 \% *$ \\
\hline Mortality ICU & $7 \%$ & $25 \% *$ \\
\hline Mortality Hospital & $13 \%$ & $39 \% *$ \\
\hline Parsonnet & $14(10)$ & $24(11)^{*}$ \\
\hline APACHE II & $19(9)$ & $23(7)^{*}$ \\
\hline \multirow[t]{2}{*}{ Ventilator Utilization } & $33 \%$ & $76 \% *$ \\
\hline & & ${ }^{*} p<.0001$ \\
\hline
\end{tabular}

Table 2

\begin{tabular}{|c|c|c|c|c|}
\hline & \multicolumn{4}{|c|}{ VENT $>2$ days } \\
\hline & \multirow{2}{*}{ No VAP } & \multicolumn{3}{|c|}{ VAP } \\
\hline & & ALL & No VAE & Yes VAE \\
\hline \# admissions & 1615 & 143 & 96 & 47 \\
\hline Age (average) ${ }^{*}$ & $69(14)$ & $71(11)$ & $70(12)$ & $72(10)$ \\
\hline LOS ICU & $16.1(38)$ & $45.9(100)^{*}$ & $47.4(122)$ & $43(26.3)$ \\
\hline LOS HOSPITAL* & $46.3(78)$ & $86.2(118)^{*}$ & $94.5(135)$ & $69.7(54.5)$ \\
\hline$\%$ ICU/Hospital Days & $39 \%$ & $54 \% *$ & $51 \%$ & $60 \% *$ \\
\hline \multicolumn{5}{|l|}{ Origin intrahospital } \\
\hline OR/PACU & $31 \%$ & $49 \% *$ & & \\
\hline TCU/Ward & $34 \%$ & $22 \% *$ & & \\
\hline \multicolumn{5}{|l|}{ Casemix } \\
\hline MED & $52 \%$ & $43 \%^{\pi}$ & & \\
\hline CARD SURG & $23 \%$ & $36 \%^{5}$ & & \\
\hline Mortality ICU & $24 \%$ & $29 \%$ & & \\
\hline Mortality Hospital ${ }^{*}$ & $39 \%$ & $42 \%$ & & \\
\hline$\%$ ICU LOS $\geq 7$ days & $67 \%$ & $98 \% *$ & & \\
\hline Ventilator Utilization & $73 \%$ & $86 \% *$ & & \\
\hline \multicolumn{5}{|c|}{${ }^{\circ} p<.001 \quad \S p=.002 \quad \pi p=.03$} \\
\hline \multicolumn{2}{|c|}{ Disposition to other ICU } & & $3 \%$ & $35 \% *$ \\
\hline
\end{tabular}

